\title{
Flexor Dysfunction Following Unilateral Transient Ischemic Brain Injury Is Associated with Impaired Locomotor Rhythmicity
}

\author{
Kiril Tuntevski \\ West Virginia University, kituntevski@mix.wvu.edu
}

Follow this and additional works at: https://researchrepository.wvu.edu/etd

Part of the Medical Biomathematics and Biometrics Commons, Medical Biophysics Commons, Medical Physiology Commons, and the Musculoskeletal, Neural, and Ocular Physiology Commons

\section{Recommended Citation}

Tuntevski, Kiril, "Flexor Dysfunction Following Unilateral Transient Ischemic Brain Injury Is Associated with Impaired Locomotor Rhythmicity" (2018). Graduate Theses, Dissertations, and Problem Reports. 3690. https://researchrepository.wvu.edu/etd/3690

This Thesis is protected by copyright and/or related rights. It has been brought to you by the The Research Repository @ WVU with permission from the rights-holder(s). You are free to use this Thesis in any way that is permitted by the copyright and related rights legislation that applies to your use. For other uses you must obtain permission from the rights-holder(s) directly, unless additional rights are indicated by a Creative Commons license in the record and/ or on the work itself. This Thesis has been accepted for inclusion in WVU Graduate Theses, Dissertations, and Problem Reports collection by an authorized administrator of The Research Repository @ WVU. For more information, please contact researchrepository@mail.wvu.edu. 


\title{
Flexor Dysfunction Following Unilateral Transient Ischemic Brain
}

\section{Injury Is Associated with Impaired Locomotor Rhythmicity}

\author{
Kiril Tuntevski \\ Thesis submitted to the School of Medicine \\ at West Virginia University \\ in partial fulfillment of the requirements for the degree of \\ Master of Science in \\ Biomedical Sciences
}

\author{
Sergiy Yakovenko, PhD - Chair \\ Xuefang Ren, MD \\ Lisa Salati, PhD \\ Department of Biomedical Sciences
}

Morgantown, West Virginia

2018

Keywords: locomotion, stroke, central pattern generation, CPG, mathematical model, holistic model, neuromuscular dysfunction, stroke sarcopenia, rehabilitation, rodent model of ischemic stroke

Copyright 2018 Kiril Tuntevski 


\begin{abstract}
Flexor Dysfunction Following Unilateral Transient Ischemic Brain Injury Is Associated with

Impaired Locomotor Rhythmicity
\end{abstract}

Kiril Tuntevski

Functional motor deficits in hemiplegia after stroke are predominately associated with flexor muscle impairments in animal models of ischemic brain injury, as well as in clinical findings. Rehabilitative interventions often employ various means of retraining a maladapted central pattern generator for locomotion. Yet, holistic modeling of the central pattern generator, as well as applications of such studies, are currently scarce. Most modeling studies rely on cellular neural models of the intrinsic spinal connectivity governing ipsilateral flexor-extensor, as well as contralateral coupling inherent in the spinal cord. Models that attempt to capture the general behavior of motor neuronal populations, as well as the different modes of driving their oscillatory function in vivo is lacking in contemporary literature. This study aims at generating a holistic model of flexor and extensor function as a whole, and seeks to evaluate the parametric coupling of ipsilateral and contralateral half-center coupling through the means of generating an ordinary differential equation representative of asymmetric central pattern generator models of varying coupling architectures. The results of this study suggest that the mathematical predictions of the locomotor centers which drive the dorsiflexion phase of locomotion are correlated with the denervation-type atrophy response of hemiparetic dorsiflexor muscles, as well as their spatiotemporal activity dysfunction during in vivo locomotion on a novel precise foot placement task. Moreover, the hemiplegic solutions were found to lie in proximity to an alternative task-space solution, by which a hemiplegic strategy could be readapted in order to produce healthy output. The results revealed that there are multiple strategies of retraining hemiplegic solutions of the CPG. This solution may modify the hemiparetic locomotor pattern into a healthy output by manipulating inter-integrator couplings which are not damaged by damage to the descending drives. Ultimately, some modeling experiments will demonstrate that the increased reliance on intrinsic connectivity increases the stability of the output, rendering it resistant to perturbations originating from extrinsic inputs to the pattern generating center 


\section{DEDICATIONS}

To health. To life.

To my family. 


\section{ACKNOWLEDGEMENTS}

This work was supported in part by IDeA CTR NIH/NIGMS U54GM104942 start-up grant to Sergiy Yakovenko, as well as NIH CoBRE P20GM109098 to James W. Simpkins.

Special thanks to the rodent surgery core led by Xuefang Ren and Heng Hu for assistance in training as well as surgical support.

Sincere gratitude goes to the Muscle Biology laboratory of Stephen E. Alway. 
List of Tables

List of Figures

List of Multimedia Files

List of Symbols and Abbreviations

1. Introduction 1

2. Review of Literature $\quad 5$

2.1. Cerebral Vascular Accidents $\quad 5$

2.1.1. Introduction to brain ischemia and reperfusion 5

2.1.2. Facts related to cerebral vascular accidents 6

2.1.3. Gait lateralization $\quad 7$

2.1.4. Experimental models of stroke induction 9

2.1.5. Shortcomings of the middle cerebral artery occlusion 10

$\begin{array}{ll}\text { model for investigating stroke-related neuromuscular dysfunction } & 10 \\ \text { 2.1.5.1 Ischemic preconditioning } & 11\end{array}$

2.1.6. Stroke affects skeletal muscle and physical activity 12

2.1.7. Neurological alterations in the central pathways lead to
maladaptation of spinal CPG centers

2.1.8. Assessment of gait deficits after stroke 16

2.1.9. Assessment of gait deficits after stroke in rodent tMCAO 17 models

2.1.10. Gait deficit assessment aids the investigations of impediments of the motor hierarchy

2.2. The Spinal Central Pattern Generator in Development 19

2.2.1. Development of the spinal interneuron population is tied to 19

retinol signaling

2.2.2. Spinal interneurons have a distinct developmental phenotype

2.2.3. Dorsal interneurons mediate somatosensory regulation of locomotion

2.2.4. Interneuronal populations in charge of central pattern generation

2.2.5. Rehabilitative strategies seek to retrain the spinal centers of pattern formation

2.3.1. The aging process affects the motor unit 31

2.4. The Sirt1/PPARy/PGC1a Axis in Aging Disorders 32

2.4.1. Sirt1 32

2.4.2. p53 33

2.4.3. PGC1a 34

2.4.4. FoxO3a 34

2.4.5. MuRF-1 35

2.4.6. MafBx/Atrogin-1 36

2.4.7. Sirt1 and p53 participate in a regulatory interplay 36 
2.5. Pathophysiology of paretic muscle after stroke 37

2.5.1. Muscle atrophy is more pronounced on dorsiflexors in
clinical observations

2.6. Mathematical evaluation of neurological deficits after stroke 39

2.6.1. Holistic modeling investigates disruption of central drives 40

2.6.2. A precise foot-placement locomotor task for the evaluation 41

3. Materials and Methods 43

3.1. Animals 43

3.2. Asymmetric Walkway 44

3.3. Middle cerebral artery occlusion - mice 44

3.4. Middle cerebral artery occlusion - rats 46

3.5. Implant design 46

3.6. Chronic electromyograph electrode implantation 48

3.7. Kinematic analysis 49

3.8. Tissue collection and storage 50

3.9. Co-immunoprecipitation and Western blotting 51

3.10. Model description 52

3.10.1. Extrinsic versus intrinsic models $\quad 54$

3.10.2. Optimization 55

$\begin{array}{ll}\text { 3.10.2.1. Optimization from solutions of hemiplegic output } & 60\end{array}$

$\begin{array}{ll}\text { 3.10.3. Output target } & 61\end{array}$

3.10.4. Stability 62

3.10.4. Statistical analysis 63

4. Results 64

4.1. Transient ischemic stroke induces dorsiflexor-dependent gait 64

4.2. Mathematical modeling predicts impairments to the central drives governing flexor function

4.2.1 Hemiplegic solutions can be numerically modified to produce healthy locomotion

4.3. Solutions of different intrinsic parameter contribution predict different modes of readaptation

4.4. Greater intrinsic parameter contribution is associated with greater stability

4.4.1. Solutions of different output are stable along different subsets of flexor and extensor related parametric pairs

4.5. Transient ischemic stroke activates multiple catabolic processes in hemiparetic dorsiflexor muscle

5. Discussion and Recommendations 94

5.1. Dorsiflexor impairment is associated with injury to the descending drives of the central pattern generator

5.2. Numerical solutions demonstrate multiple strategies for solving locomotor output 
5.3. Numerical solutions predict flexor integrator activity as the culprit of foot-drag in hemiparetic locomotion

5.3.1. The hemiparetic strategy lies close to an alternative solution space of healthy locomotion

5.4. A precise foot-placement task requires proper modulation of the descending drives

101

5.4.1. Intrinsic parameters stabilize the locomotor output

102

6. Supplementary Figures

104

7. References

108

8. Appendix

123 


\section{LIST OF TABLES}

Table 1. Summary of locomotor conditions. 43

Table 2. Parameter nomenclature. $\quad 55$

Table 3. Model types. 56

Table 4. Alternate parametric subgroups. $\quad 62$ 


\section{LIST OF FIGURES}

Figure 1. Motor control hierarchy. 3

Figure 2. Spinal cord interneurons are derived from specific mitotic progenitor 14 zones.

Figure 3. Interneuronal connections have distinct innervation modes. 15

Figure 4. Summary of spinal cord motor neuronal and interneuronal development. $\quad 23$

Figure 5. $\quad$ Asymmetric walkway model. $\quad 42$

$\begin{array}{lll}\text { Figure } 6 . & \text { Implant design. } & 47\end{array}$

$\begin{array}{lll}\text { Figure 7. Implant assembly. } & 49\end{array}$

Figure 8. Connectivity scheme of single and multiple integrators. 52

$\begin{array}{lll}\text { Figure 9. Optimization. } & 57\end{array}$

Figure 10. Initial conditions $\quad 59$

$\begin{array}{ll}\text { Figure 11. Analysis scheme } & 60\end{array}$

Figure 12. The asymmetric walkway distinguishes lateralized gait. 65

Figure 13. In vivo electromyographic (EMG) recordings of symmetric and 66 asymmetric locomotion.

$\begin{array}{lll}\text { Figure 14. } & \text { Analysis of asymmetric measures. } & 66\end{array}$

Figure 15. Locomotor phase characteristics after transient unilateral ischemic brain $\quad 67$ injury.

Figure 16. In vivo electromyographic recordings from the precise foot placement $\quad 67$ locomotor task.

$\begin{array}{lll}\text { Figure 17. Intralimb coordination. } & 68\end{array}$

$\begin{array}{lll}\text { Figure 18. } & \text { Output of simulated locomotor behavior. } & 70\end{array}$

Figure 19. Cost reduction by the Nelder-Mead method. 71

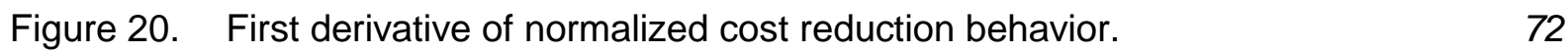

Figure 21. Different initial conditions take different paths through the task-space of 73 cost optimization.

Figure 22. Bootstrap analysis of solution sets reproducing healthy locomotion. $\quad 73$

$\begin{array}{lll}\text { Figure 23. } & \text { Analysis of solution sets reproducing hemiparetic locomotion. } & 74\end{array}$

Figure 24. Statistical analysis of parametric weight distribution between readapted 76 solutions from hemiplegic IC.

Figure 25. Parametric weight distribution differences between $\mathrm{IC}^{S}$ and $\mathrm{IC}^{\mathrm{F}} \quad 78$ solutions.

Figure 26. Solutions of different intrinsic parameter contributions have different manners of predicting left hemiparetic modifications of the central pattern generator model

Figure 27. Introduction of noise to parametric subsets. 85

Figure 28. Stability and velocity analysis of four different healthy solutions. 86

Figure 29. Error change is distributed among flexor and extensor integrator related 90 parametric subsets.

Figure $30 \quad$ Solution space relationships between different pairs of extrinsic and intrinsic $\quad 91$ parameters for single half-center output.

Figure 31 The cell stress pathway demonstrates preferential dorsiflexor impairment in 93 hemiplegic muscle after ischemic stroke. 
Figure S1 Single parameter analysis for parametric alteration between healthy left- 104 preferred and right-preferred gait.

Figure S2 Parametric alteration trends from single parameter analysis of solutions of 105 varying intrinsic parameter contributions.

Figure S3 Logarithmic error differences between optimized and suboptimal 106 solutions under 10 SNR, and in relation to Figure 29.

Figure S4 Root-mean-square differences between optimized solutions and 107 suboptimal solutions from Figure 30 . 


\section{LIST OF MULTIMEDIA FILES}

Multimedia File 1. CPG7a.m

Multimedia File 2. CPG7a_optimize.m

Multimedia File 3. Statistical_Analysis.m

Multimedia File 4. Cranial_Implant.skb 
LIST OF SYMBOLS AND

ABBREVIATIONS

CNS - central nervous system

BBB - blood-brain barrier

BBB Scale - Basso-Beatie-Bresnahan

locomotor evaluation scale

DNA - deoxyribonucleic acid

Sirt1 - sirtuin 1

C56BL-7 - C56 Black-7 mouse line

CVA - cerebral vascular accident

tMCAO - transient middle cerebral artery

occlusion

TPA - tissue plasminogen activator

ROS - reactive oxygen species

CCA - common carotid artery

ICA - internal carotid artery

ECA - external carotid artery

TTC - triphenyltetrazolium chloride

$\alpha \mathrm{MN}$ - alpha motor neuron

IN - interneuron

CPG - central pattern generator

EMG - electromyogram

$\mathrm{RA}$ - retinoic acid (vitamin A)

$\mathrm{RE}$ - retinyl ester

RBP4 - retinol binding protein 4

Raldh - retinaldehyde dehydrogenase 1-3

RARE - retinoic acid receptor element

Hox - homeobox gene

RAR - retinoic acid receptor

$\mathrm{RXR}$ - orphan retinoic acid receptor

HDAC - histone deacetylase

VEGF - vascular endothelial growth factor

Lgl1 - late-gestational lung 1

RNAPII - RNA polymerase II

Prep1 - Pbx-regulating protein 1
Pbx1 - pre-B cell leukemia factor protein

Onecut1(HNF-6) - one cut homeobox 1

(hepatocyte nuclear factor 6)

Meis2 - Meis1 homolog homeobox protein 2

Irx - iroquoix

Hoxa2 - homeobox A protein 2

Hoxb1/2 - homeobox B protein 1/2

Mafb - V-maf musculoaponeurotic fibrosarcoma oncogene homolog $B$

Etv4 - ETS translocation variant 4

Zpfm2 - aka Fog2

Znf503/Nolz1 - zinc finger protein 503

$\mathrm{HH}$ - Hamburger-Hamilton stage of

development

pMN - pre-motor neuron mitotic zone

p0-p3 - pre-ventral interneuron mitotic zone

Dbx1 - developing brain homeobox 1

Evx1 - even-skipped homeobox 1

V0-V3 - ventral interneuron varicosities 0-3

RC - Renshaw cell

GABA - gamma aminobutyric acid

En1 - engrailed 1

Gata2/3 - GATA binding protein 2/3

Chx10 - ceh-10 homeodomain-containing

homolog 1

Hb9/MNR2 - hybrid 9/motoneuron 2

TGF- $\beta$ - transforming growth factor beta

TIF1 $\beta$ - transcription intermediary factor $1-\beta$

BMP 4-7 - bone morphogenic proteins 4-7

BmpR1a/b - BMP receptor $1 \mathrm{a} / \mathrm{b}$

Math1/Atoh1 - protein atonal homolog 1

Ngn1/2 - neurogenin 1/2

Mash1 - Ascl1 (ascaete-scute homolog 1)

Shh - Sonic hedgehog

Nkx - nkx homeodomain factor

Olig2/3 - oligodendrocyte transcription factor $2 / 3$ 
Gsh1/2 - glutathione synthetase 1/2

Ascl1 - Mash1 (ascaete-scute homolog 1)

Pax6 - paired box 6

Lhx3 - LIM homeobox 3

Pbx1 - pre-B cell leukemia 1

Pbx3c - pre-B cell leukemia 3, subtype c

e1-18.5 - embryonic days 1-18.5

pd1-6 - dorsal interneuron 1-6 mitotic

varicosities

pdIL - dorsal late developing interneuron mitotic varicosities

DMRT3 - doublesex and Mab-3 related

transcription factor 3

Pax3/7 - paired box 3/7

Shox 2 - short stature homeobox 2

CS- $\alpha M N$ - corticospinal to alpha-motor neuronal tract

$\mathrm{MHC}$ - myosin heavy chain isoforms

EPSP - excitatory post-synaptic potential

ATPase - adenosine triphosphatase

$\mathrm{Hz}-$ hertz

PPARY - peroxisome proliferation activated

receptor gamma

PGC1 1 - PPAR-gamma co-factor 1 alpha

p53 - $53 \mathrm{kDa}$ protein involved in the DNA

damage response and $\mathrm{G} 1$ cycle arrest pathway

FoxO1 - forkhead box 1

FoxO3a - forkhead box 3a

FoxO4 - forkhead box 4

IPO 7/8 - nuclear importin 7 and 8

MuRF-1 - muscle-specific RING B-box coiled-

coil 1 E3 ubiquitin ligase

MuRF-2 (TRIM55) - MuRF isoform 2

MuRF-3 (TRIM54) - MuRF isoform 3

GMEB-1 - glucocorticoid modulatory element

binding protein 1
MafBx/Atrogin-1 - muscle-specific F-box protein (atrophy gene 1)

p38 MAPK - $38 \mathrm{kDa}$ protein mitogen-activated

protein kinase

elF-3 - eukaryotic initiation factor complex 3

LZ - leucine zipper domain

LCD - leucine charged residue rich domain

Dvl - disheveled protein

PAK1 - p21(RAC1) activated kinase 1

TA - tibialis anterior muscle

GA - gastrocnemius muscle

PAL/PA - palmaris longus muscle

EDC - extensor carpi digitorum

ECR - extensor carpi radialis

$\mathrm{SOL}$ - soleus

$\mathrm{VM}$ - vastus medialis

$\mathrm{VI}$ - vastus intermedialis

$\mathrm{VL}$ - vastus lateralis

$\mathrm{RF}$ - rectus femori

M1 - primary motor cortex

ISL - interstride length

$\mathrm{SL}$ - stride length

IC - initial conditions

$I^{S}$ - symmetric initial conditions

$I^{F}$ - flexor-biased initial conditions

$\mathrm{IC}^{\mathrm{HS}}$ - hemiplegic initial conditions from

symmetric optimization solutions

$\mathrm{IC}^{\mathrm{HF}}$ - hemiplegic initial conditions from flexor-

biased optimization solutions

PLA - polylactic acid

$A l_{h}$ - horizontal asymmetry index

$A I_{v}$ - vertical asymmetry index

$E C L$ - enhanced chemiluminescence lighting 


\section{Introduction}

It has been postulated that movement is one of the defining parameters of life. Furthermore, it has become regularly accepted that movement represents one of the key drives of animal, and particularly, mammalian life, to the point that the largest and most significant portions of the central nervous systems of almost all life forms is represented by the somatosensory and motor systems ${ }^{1}$. As such, the motor cortex of mammals has evolved to accommodate these two systems in separate anatomic locations, allowing for functional segregation for the purpose of protection of function. This functional segregation imposes demands for improved communication, faster perception, and more efficient control of all parameters of movement. The central nervous system, therefore requires heavy involvement of all aspects of homeostasis, nutrition, and maintenance. The CNS of higher organisms, therefore, requires a balance of all aspects of organic complexities, and systemic contributions in order to achieve energetic homeostasis. These aspects include, but are not limited to, oxygenation, glucose and amino acid supply, as well as immunological balance involved in clearance and repair of damage induced by toxins, as well as the clearance of aberrant connectivity produced by mispairing and mismatching of neural impulses. Further, this correct pairing of neurons involved in somatosensory and motor commands needs to be achieved through proper production and maintenance of neurotransmitters necessary for proper function, long and short term modification of synapse strength, as well as proper impulse summation necessary for balanced network activity and efficient learning of novel tasks.

These networks, throughout the complexity of the central nervous system, navigate highly organized state space (elsewhere task space), which imbues the connected network with computational capacity. This computational capacity represents the core material of learning and modification of learned strategies, and neuronal network homeostasis is necessary in order to maintain the learning capacity of the network, as well as to eliminate undesired pairing, and to fine-tune the network into proper function. 
The neuromuscular system in higher mammals is comprised of a complex architecture which requires a tightly regulated system of homeostatic regulation in order to be properly developed and maintained (Figure 1). These homeostatic mechanisms become imbalanced upon injury, producing deficits in the production and execution of motor commands, which clinically represent one of the major healthcare burdens. For that purpose, experimentalists have generated multiple tools to evaluate motor deficits following injury of the central nervous system. The first part of this thesis focuses on the development of the spinal central pattern generator for locomotion with respect to the various homeostatic events which occur in the process of intrauterine as well as postpartum growth of the organism. In the second portion, this thesis will focus on experimental methods of evaluation of motor deficits, as well as some of the deficiencies induced by experimental models of injury in animal systems. These novel methods of evaluation of motor deficits serve as useful tools in the evaluation of the corticospinal mechanisms involved in the investigation of the locomotor system in mammals. Specifically, a novel visuomotor task of precise foot placement, and under the hypothesis that precise foot placement preferentially requires the participation of higher centers of motor regulation, will demonstrate that the phase characteristics of stance and swing are altered following ischemic brain injury. Then, a holistic Sherrington-Brown based model of locomotion will demonstrate that under certain initial conditions, the predicted deficits demonstrate a more deleterious effect on the centers governing flexor function, which ultimately coincides with the clinically observed flexor deficits. These deficits have been well documented historically in clinical as well as experimental settings of investigation of stroke. These impairments to the central drive for locomotion are associated with a clinical picture featuring atrophying muscle on the hemiparetic side, with a bias to the flexor muscle population over the atrophy of plantar flexors. This is not surprising, as plantar flexor activity in posture and locomotion is reflexive and does not necessarily require the input of the descending drives originating in higher centers of the central nervous system. 
A.

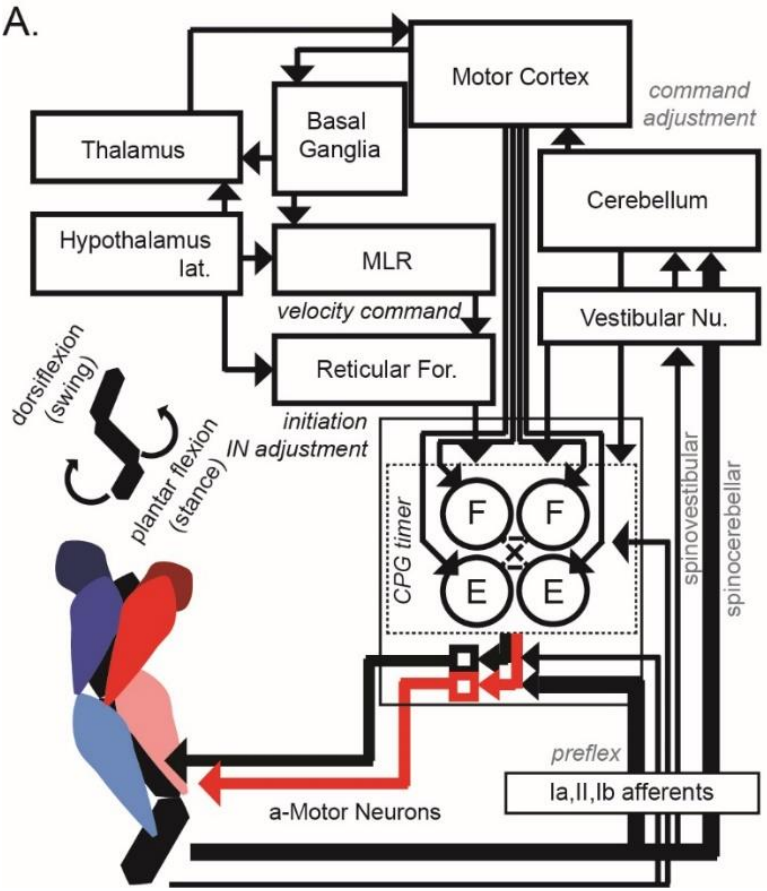

B.

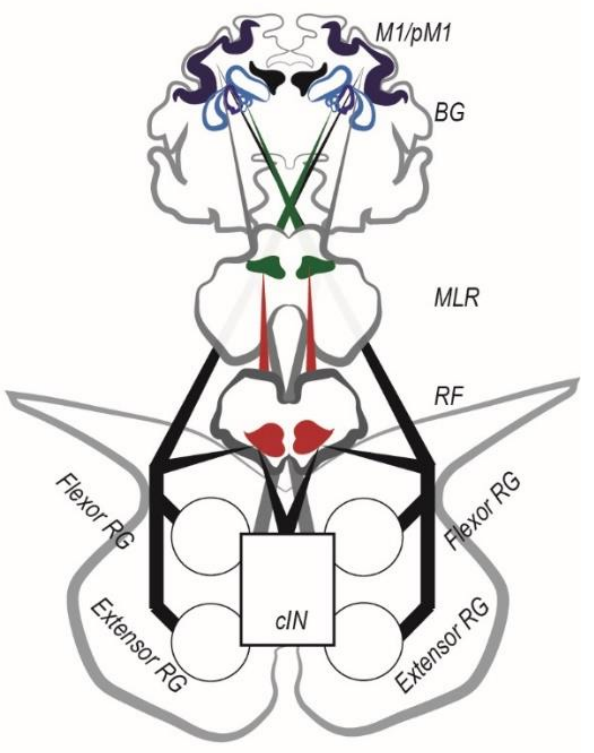

Figure 1. Motor control hierarchy. A. Descending and ascending inputs converge on the spinal centers of pattern generation. This pattern forming layer has been implicated in mediating a wide repertoire of tasks, ranging from reaching and precise dexterity tasks, to proper functioning of the central pattern generator timer for locomotion. Descending corticospinal tracts from the motor cortex, cerebellum and vestibular nuclei converge on interneuronal populations in the spinal cord, and assist the definition of task-space, as well as adjustment to changing demands by the environment. Tonic input from the midbrain locomotor region and reticular formation regulate the velocity of extensor related stance and flexor related swing phases for the purpose of locomotion. The interconnectivity between higher centers, such as the thalamus, hypothalamus and basal ganglia interplay to regulate the recruitment and refinement of muscular synergistic behavior defined by the tasks space. Ultimately, the spinal interneuron populations regulate the firing of $\alpha$-motor neurons, which act as the actuators of muscular function. In-set - dorsiflexor dependent swing and plantar flexor stance phases are performed by diverging sets of motor neuronal populations. B. The most relevant centers for the purpose of this modeling study are the motor and premotor cortices, which are hypothesized to play a primary activation, as well as modulatory function of constant descending drives which originate in the midbrain locomotor area (MLR). This center projects contralaterally to the postcommissural reticular formation, which then produces the primary activation input to drive locomotion even in decorticate and decerebrate preparations. Corticospinal pathways cross at the posterior commissure and modify the tonic input of the ipsilateral local, intersegmental and spinal commissural interneuronal populations, which ultimately set the rhythmicity of the locomotor pattern.

Moreover, the atrophy response seems to affect the number of slow type I fibers, which is a paradoxical find since these fibers are predominately atrophy resistant. As such, the atrophy response resembles the one seen in dystroglycanopathies and muscle tyrosine kinase- 
associated myasthenic syndromes, rather than the atrophy response observed in spinal cord injury or denervation. Interestingly, the DNA damage response pathway, which participates in the preservation of surviving motor units in various forms of muscular dystrophies as well as spinal cord injury, may also play a crucial role in the preservation of the motor units after ischemic brain injury. Impediments of this pathway in muscle, and in response to CNS damage, is characteristically associated with atrophying muscle, but may provide insights into the spinal actuators which become injured upon classical transient ischemic brain injury. Interestingly, this pathophysiological state of hemiparetic muscle is stereotyped to the paretic dorsiflexor population, which ultimately is identified as the culprit of many of the maladaptation associated with limb progression during locomotion after stroke. 


\section{Review of Literature}

\subsection{Cerebral vascular accidents}

One of the most neurotoxic conditions arises due to cerebral vascular accidents (CVA). CVAs represent the third leading cause of death in the developed world and the fifth costliest condition to rehabilitate. Stroke-related sarcopenia and weakness cause serious detriments to the neuromotor system, whereby rehabilitation aimed at improving functionality represents a major portion of the total cost associated with post-stroke rehabilitation². Specifically, up to two thirds of all patients retain disability, half of them show signs of hemiparesis, and a third of all patients are unable to walk without disability ${ }^{3}$. The nature of neurological disfunction has been the target of many research attempts, and it is widely accepted that ischemia alone, elsewhere ischemiareperfusion induced brain injury, initiates resident immune as well as intrinsic cellular necrotic pathways that lead to neuronal cell death, pro-inflammatory responses, and induce macrophage activity and clearance of damaged tissue. This inevitably leads to homeostatic impairments in higher brain centers governing motor function. For the purpose of this text, hemiparetic and paretic limb or muscle will be the term reserved to indicate the limb or muscle from the affected side. In this text, furthermore, it is taken as obvious that a right-sided stroke, causes deficits on the left side, following the rules of opposition.

\subsubsection{Introduction to brain ischemia and reperfusion}

Ischemia is the general condition in which cells undergo pathologic alterations to cellular metabolism associated with the cessation of oxygen supply and can be induced either by blood flow restriction, accumulation of blood or cerebral edema due to various etiology. The most prevalent actors initiating of such cerebral vascular accidents can be lipidemic, proinflammatory, thrombotic, or can be due to physical trauma ${ }^{4}$, which results in vessel occlusion and subsequent abrogation of transport of nutrients and essential gasses. A transient ischemic stroke occurs when the vessel occlusion is temporary, most commonly involves the middle cerebral artery or one of 
its branches, which is followed by a subsequent event of nutrient and oxygen resupply, termed reperfusion. Most commonly, this condition is mimicked experimentally by the transient middle cerebral occlusion model (MCAO, elsewhere tMCAO). The clinical significance of this model has been discussed elsewhere ${ }^{5}$, and in its classical experimental form it actually represents a minority of cases, as clinically the reperfusion event is most commonly caused by tissue plasminogen activator (TPA) therapy, which is rarely used due to temporal restrictions (administered within 6 to 12 hours after diagnosis of an ischemic stroke $)^{6}$. It can, however, model some of the proinflammatory transient types, hence its widespread use in experimental models ${ }^{7}$. There seem to be some differences in the pathology of the permanent ischemic and the ischemia/reperfusion types of stroke. Though reperfusion restores nutrient supply to the ischemic tissue, it rapidly oxygenates the sickly penumbra, which leads to further toxicity termed reperfusion-induced injury ${ }^{8,9}$, induced by accumulation of reactive oxygen species ${ }^{10}$, which is reversed by mild $\operatorname{acidosis}^{11,12}$.

\subsubsection{Facts related to cerebral vascular accidents}

According to some reports, cerebrovascular accidents (CVA) are the second leading cause of death worldwide, trumped only by the number of deaths due to cardiovascular disease ${ }^{2}$. This term is used more widely to capture the various etiologies associated with stroke. Unfortunately, stroke is not a condition caused by a single etiology, but a multifactorial pathological state not limited to obesity, insulin insensitivity, chronic and acute stress, elevated blood pressure, and chronic and acute inflammation and clotting conditions ${ }^{4}$. Yet, the execution of disease due to all of these factors is commonly recognized as localized or generalized brain injury due to restriction of supply of oxygen and glucose. This restriction is causatively elicited either by insipid vasospasm, thrombotic or lipidemic vessel occlusion, or vessel permeation leading to edema or hemorrhage. A large majority - about $85 \%$ - of all strokes are ischemic, which is significantly less severe than the hemorrhagic counterpart ${ }^{3}$. The ischemic injury is further compounded by a paradoxical 
reperfusion injury upon reestablishment of proper blood circulation through the affected brain regions. This reperfusion injury is classically thought to be instigated by $\mathrm{Ca}^{2+}$ mishandling and its accumulation in damaged mitochondria. This insult further accrues reactive oxygen species (ROS) in a feedforward etiologic process known as ROS-induced ROS release ${ }^{9}$. In both the ischemic and hemorrhagic form, the exacerbation of the condition is brought about by anterograde Wallerian degeneration of white and gray matter tracts on the affected side due to aberrant activation of proinflammatory and proapoptotic pathways by factors present in blood, such histamine, serotonin, cytokines and proteases released by activated central and peripheral cells of the immune system ${ }^{13}$.

\subsubsection{Gait lateralization}

As movement represents one of the key postulates of all life, higher order animals have developed a numerous set of repertoires necessary for performing complex tasks, such as predation, predatory evasion, exploration, feeding and reproduction. In mammals, the complexity of the neural hierarchy allows for greater precision of movement control. This hierarchy is distributed in centers described in later chapters. There are several mechanisms controlling muscular synergies which are required for stable execution of locomotor tasks. At the level of spinal motor neuron recruitment, which ultimately drives muscular contraction and movement at the level of the segments, the coordinated coactivation is driven by intermediate neuronal networks, termed interneurons, which regulate the synergistic firing of the a-motor neurons. This repertoire of cocontraction ultimately determines the rhythmicity of stride essential for locomotor execution.

The modification of this rhythmicity in quadrupedal animals generates various forms of gait, such as trotting, cantering, flying ambling, etc. Yet, the propriospinal networks are merely permissive of such function, while the control and regulations of these repertoires is believed to be represented at the level of the motor cortex, subcortical centers such as the lenticular nuclei, as well as the cerebellum (which is ultimately tasked with optimization of locomotion and movement 
in general). These alterations in gait rhythmicity can be monitored using electromyography as well as other forms of telemetric analysis of multisegmented dynamics during daily movement activities.

Historically, there have been many studies using electromyography to investigate locomotion, and it generally involves either non-invasive procedures, such as pasting extracutaneous electrodes for monitoring muscular activity in humans and animals, or uses invasive transcutaneous or intramuscular implants of electrodes, more generally reserved for animal models. In more recent studies, it has become common to use other non-invasive methods of gait assessment, such as telemetry, in order to provide cheap and relatively harmless means of performing assessment of a patient's impairment. These approaches enable a clinician to monitor a patient's palliative progress remotely, and promises to outsource a great deal of heal-care burden onto automation.

For experimental models of gait assessment in animal models, gait assessment can be performed by using one of several commercially available devices. The implementation of force-sensor analysis on rat locomotion has revealed that arrangements that impose gait lateralization can be a useful tool in the analysis of gait deficits, and can replace invasive intramuscular implantation commonly used to study synergistic coactivation of muscles. For that purpose, telemetric analyses of rats which have suffered a transient ischemic brain injury event reveal that the impairment of rhythmicity of locomotion can be used to reveal underlying neurological deficits by using holistic models of central pattern generation. These holistic models are designed in such a way that the oscillatory activity of flexor and extensor motor neuron populations, represented by the swing and stance phase of locomotion, are related through underlying neural networks. Such models do not use cellular and neuronal models of coupling, but in stead use arbitrarily defined networks which are illustrative of the general connectivity of the oscillatory centers. 


\subsubsection{Experimental models of stroke induction}

As cerebrovascular accidents are not necessarily caused by a singular risk factor, there have been many experimental surgical methods of induction of stroke in animal models to account for the various confounding factors leading to ischemia ${ }^{5,6}$. These models include mimicking stroke through generalized vessel occlusion, hypoxia induced by hypovolemia or hypotension, as well as the middle cerebral artery occlusion model (MCAO). Experimental methods that induce hypovolemia or hypotension cause generalized hypoxia that affects the entire brain, and translationally capture only a small fraction of strokes. Furthermore, acute unilateral common carotid artery (CCA) or internal carotid artery (ICA) ligation does not result in significant ischemia as circulation to the middle cerebral arteries can be supplied bilaterally by a single carotid artery through a unique artery-to-artery invasion known as the Circle of Willis. Older models of autologous thromboembolic stroke had been done in larger mammals such as dogs and pigs during the early periods of investigation, but involved transcranial injection of thrombi of unstandardized size into the arterial branches of the MCA. Depending on the size of the occluded vessel, these maneuvers produced varying results. Since the model has transitioned to mice and rats, it has been somewhat improved to involve catheterization of the ICA and injection of the clots to the MCA without craniotomy. In recent years, these models are generally reserved for investigating coagulation and thrombolysing properties of various medication ${ }^{14}$.

As the transient MCAO ( $\mathrm{MCAO}$ ) model mimics the etiology of the ischemia well, induction of ischemia through the MCA remains the most popular method of induction of stroke to date. It is performed by the proximal occlusion of the middle cerebral artery by filament insertion through the internal carotid artery. Once the occlusion of the middle cerebral artery is achieved, the

duration of ischemia can be easily controlled for duration and extent of occlusion, usually measured using a portable Doppler sonograph. After the vessel has been occluded for the desired duration, the silicone-coated filament is removed and circulation to the MCA restored. The 
materials for the execution of the procedure are relatively cheap and readily available, and the embolization probes come in customizable sizes carefully adjusted for the appropriate rodent model. It has also been common practice for labs to generate their own monofilament probes by either melting or burning 6.0 monofilament sutures to the desired diameter ${ }^{15}$. Generally speaking, a filament diameter of $0.23 \pm 1 \mathrm{~mm}$ and $9-10 \mathrm{~mm}$ long silicone coating generates reproducible ischemia in adult mice weighing between 26 and $34 \mathrm{~g}$, if inserted into the lumen of the ICA to the $10 \mathrm{~mm}$ mark. Furthermore, this model is technologically less demanding, as it does not involve catheterization unlike some of the more novel autologous thromboembolic models that rely on exogenous generation of thrombotic clots and poorly controlled duration of ischemia.

In the realm of tMCAO surgeries, we recognize two main methods of induction of occlusion - the Koizumi and Longa methods ${ }^{16}$. Essentially, these methods are very similar due to the fact that they both induce a transient occlusion into the MCA through cannulating the ICA, and do not involve craniotomy. The two methods differ in the location of the arteriotomy - a necessary step for catheterization and embolization. Where the Koizumi method involves filament insertion through the CCA, the Longa method involves insertion through the external carotid artery (ECA), whereby the CCA is kept intact upon reperfusion. While the Longa method is physiologically more favorable for studies requiring reperfusion through both the left and right CCAs, the Koizumi method does not fall behind in applicability, as ligation of the CCA does not cause significantly different ischemic/reperfusion damage due to open circulation through the Circle of Willis.

\subsubsection{Shortcomings of the middle cerebral artery occlusion model for investigating stroke-related neuromuscular dysfunction}

Both methods have a potential shortcoming in regards to the investigation of stroke-induced sarcopenia, which may influence the function of the locomotor system at lower control centers such as directly affecting muscle function. Namely, though normal thyroid function is also necessary for maintenance of muscle mass ${ }^{17}$, both methods require the permanent ligation of the 
ECA. As the superior thyroid artery (STA) originates close to the proximal base of the ECA, one of the lobes of the thyroid gland will remain under lowered blood supply, and will be functionally invaded by the inferior thyroid artery alone. The blood supply to the other lobe of the thyroid will remain intact and its function preserved. The sham operation for these experiments may involve the permanent ligation of the ECA, so any confounding factors conferred by thyroid insufficiency will be properly controlled for.

Finally, though stroke sarcopenia in patients has been documented well, and demonstrated in animal models of $\mathrm{tMCAO}^{18}$, direct investigation is lacking for the chronic occlusion and hemorrhagic models. There is no doubt these models of cerebral vascular damage produce a form of sarcopenia of their own as they are significantly more detrimental to the neuronal tissue, this issue lacks direct evidence. Since the reperfusion event alone is a cause of exacerbation of infarct volume (in fact, triphenyltetrazolium chloride - TTC - staining of the penumbra might not even be evident without at least 60 minutes of reperfusion), it is unclear whether the chronic occlusion model will produce the same extent of muscle atrophy without the reperfusion injury. It would be imperative to compare the two models before switching from a transient to a permanent occlusion model.

\subsubsection{Ischemic preconditioning}

Ischemic preconditioning is a phenomenon whereby brief transient exposures to ischemia provide a protective effect in subsequent prolonged periods of ischemia. This phenomenon has been described in the heart, as well as the brain, though no clear mechanism of its action has been proposed to date. There have been some studies that suggest that ischemic preconditioning may be influenced, at least in part, by an increased capacity of the electron transport chain complexes after brief periods of hypoxia. Should this evidence be considered, it would seem that it is mitochondrial dysfunction that initiates the proapoptotic and proautophagic pathways during periods of reperfusion that are responsible for at least some neuronal death, and may be mediated 
in part by lowered activity of mitochondrial complex $\left.\right|^{19}$. Exercise has been shown to exhibit some protective effects on skeletal muscle, as well as in heart, enabling these tissue types to develop a kind of temporary resistance to hypoxia. In fact, it has been proposed that spontaneous vessel occlusion and reperfusion in patients with predisposition to cardiac ischemia may serve a source of natural protection against hypoxia. This phenomenon is not, in fact, flawless, as it does not work for many of the hemorrhagic cases, which still represent at least $20 \%$ of all cerebral vascular events. Furthermore, the predisposing factors leading to vessel occlusion will eventually lead to total occlusion without subsequent reperfusion, which renders the process essentially inefficient.

\subsubsection{Stroke affects skeletal muscle and physical activity}

It is likely that this imbalance of excitation and inhibition also causes changes in the potentiation and firing properties of the motor neurons themselves, ultimately leading to an impaired lower motor neuron recruitment which is remarkably biased towards the paretic side, inducing spasticity, high fatigability, and low motor unit recruitment rate 20,21 . A hyperactive clonus has sometimes been hypothesized to be induced by the seemingly chaotic reorganization of the supraspinal centers' patterning spinal structure, and haphazard pairing of the different interneuronal types driving type I and type II fibers with their presynaptic centers ${ }^{22}$. This aberrant pairing is associated with some aMN death, resulting in a decreased number of active motor units ${ }^{23}$, and produces a significant loss of muscle and skeletal mass on the paretic side ${ }^{24}$. The number of rescued motor units is ultimately predictive of recovery ${ }^{25}$. As all of these neural correlates are the targets of

rehabilitative strategies involving exercise ${ }^{26}$, perhaps strategies aimed at minimizing damage to the motor unit, may also stabilize the underlying aMNs, thereby increasing the efficacy of the therapeutic process. 


\subsubsection{Neurological alterations in the central pathways lead to maladaptation of spinal CPG centers}

The deficits associated with stroke are undoubtedly related to the ischemic affect over individual or groups of neurological centers that govern behavior. By that token, an ischemic insult to Brocca's or Wernicke's area will result in speech formulation and execution, and an ischemic insult to the motor cortex governing forelimb function will result in motor affects on the contralateral limb. In the event of insufficient intervention, the ischemic insult becomes permanent, leaving the affected brain area scarred and dysfunctional. Moreover, impairments of the central nervous system often result in other permanent alterations of the affected motor actuators, resulting in muscular as well as bone deficits on the hemiparetic side ${ }^{24}$. Historically, early interventions have been thought to be most effective. Such interventions aim at restoring motor function by retraining the affected individual to perform every-day tasks, often by employing alternative multisegmented strategies for motor performance ${ }^{22}$.

The development of motor deficits after unilateral brain injury is not always uniform. In the beginning phases of injury, there is a marked hyporeflexia associated with upper motor neuron (layer 5 of the primary motor cortex) injury ${ }^{27}$. Paradoxically, about a third of patients exhibit hyperreflexia and spasticity, which is defined as the reflexive resistance to tonic stretch ${ }^{28}$. This phenomenon is undoubtedly associated with maladaptation of the central spinal centers to the absence of tonic input originating from the cortex.

As it is known that spinal locomotor central pattern generation is mediated by propriospinal interneuron populations, this raises the question about the stress on the descending corticospinal tracts, and the role that impairments of the function of corticospinal tracts might have on spinal centers of rhythmogenesis. Conspicuously, corticospinal connectivity to the $\alpha$-motor neurons in most mammals is rather scarce. Instead, the modulators of the interneuronal activity is most probably the reason behind the production of sub-optimal tasks-space solutions with dysfunctional 
outputs identified in hemiplegic reaching and locomotion. These corticospinal tracts greatly innervate the ventral V0, V1, V2 and V3 subpopulations of interneurons, and exhibit potent modulation on the dorsal interneuronal dl1-dl6 populations (Figures 2 and $\mathbf{3})^{29}$. Furthermore, inputs from the reticular formation also project on interneuronal populations in the spinal cord ${ }^{30,31}$, and most probably regulate the rhythmic oscillatory states at different locomotor speeds (Figure 3). The reticular formation along with a center termed the midbrain locomotor region (MLR) have been implicated as crucial drivers of spinal rhythmogenesis at the turn of the century, when it was demonstrated that tonic inputs from these centers are sufficient to induce locomotor behavior even in higher mammals ${ }^{32,33}$. The ventral subpopulations of interneurons are known to directly mediate the generation of locomotor output, as many studies in neonatal rodent spinal cord explants have demonstrated, and several cellular mathematical models of left-right coordination have demonstrated ${ }^{34}$. These networks have multiple manners of function, which is dependent on interneuronal connectivity architectures ${ }^{35-37}$.

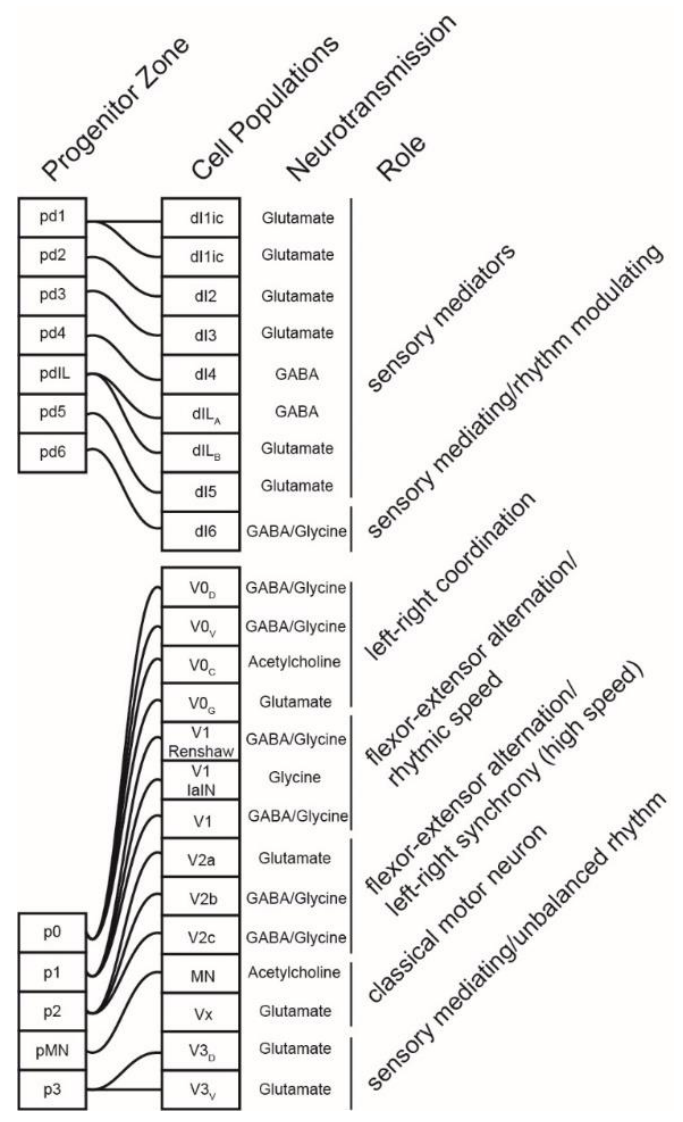

Figure 2. Spinal cord interneurons are derived from specific mitotic progenitor zones. Dorsal interneurons, marked d1-d6, are derived from dorsal progenitor zones (left), marked pd1-pd6 (top), while ventral interneurons and motor neurons are derived from ventral progenitor zones, marked p0-p3 (bottom). Each interneuronal cell population type (second column from left) has a specific excitatory or inhibitory mode of neurotransmission (second column from right). The neurons derived from ventral progenitor zones are generally thought to be responsible for generating locomotor output, while the neurons derived from the dorsal progenitor zones are thought to play a modulatory function, mediating tactile as well as proprioceptive feedback onto motor interneurons derived from the ventral progenitor zones (right column). Combined data from Alaynick et al (2011), Gosgnach et al. (2006), Gosgnach S. (2011), and Dyck et al. (2012) 
A.

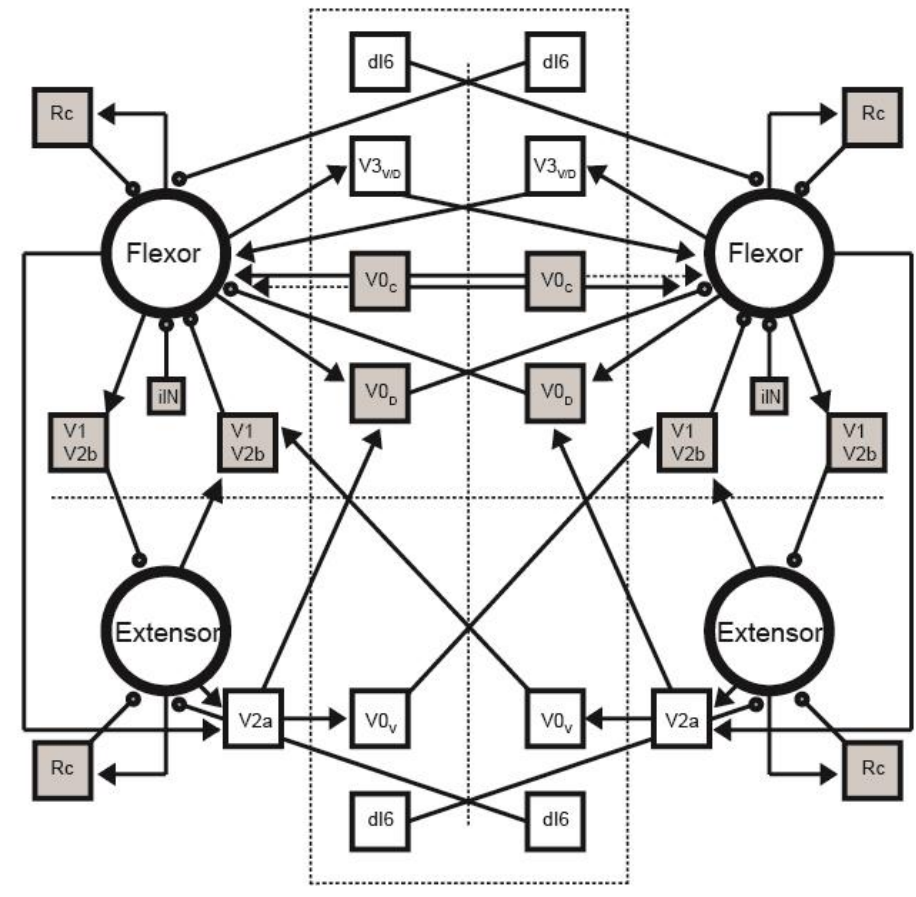

B.

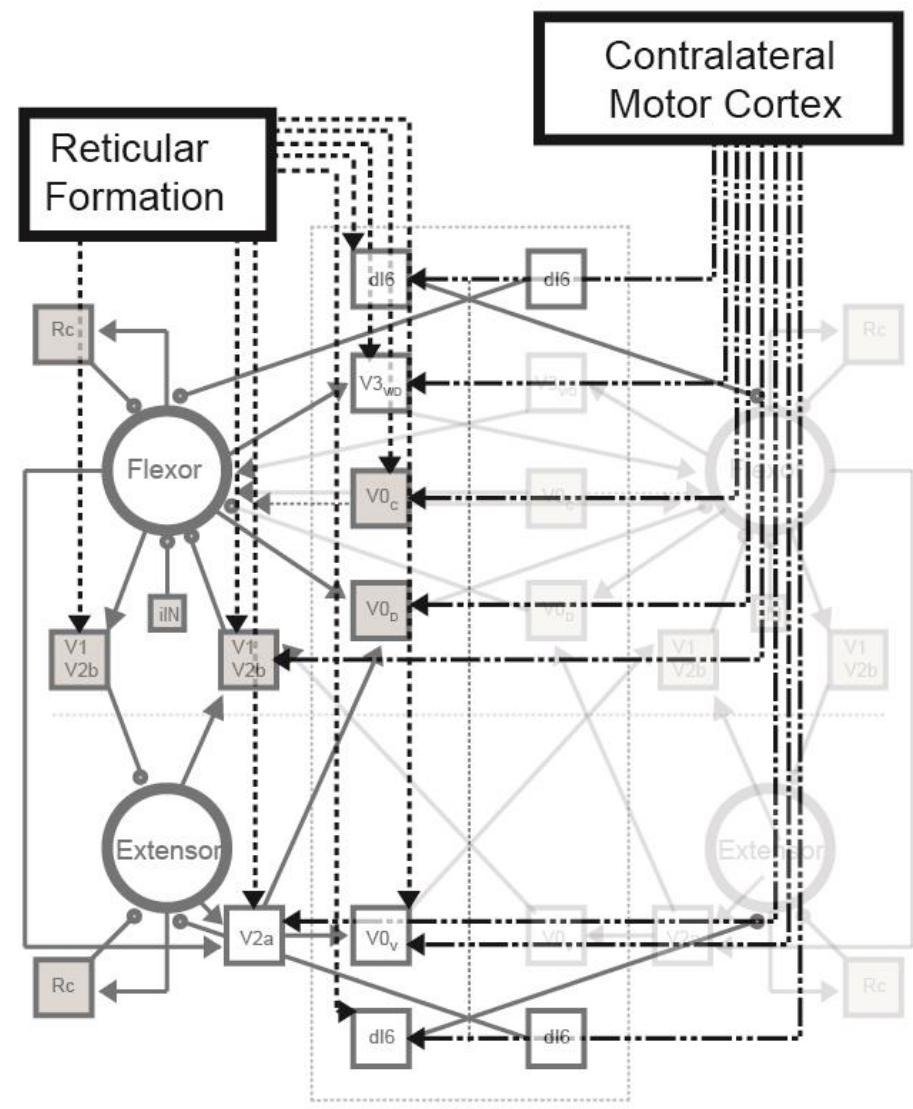

Figure $3 . \quad$ Interneuronal connections have distinct innervation modes. A. An expanded interneuronal connectivity scheme from data published by N. Shevtsova et al. (2015) (combined with data from J. Zhang et al. 2014, and Alaynick et al. 2011). The commissural and ipsilateral connectivity of spinal centers responsible for central pattern are interdependent. B. The corticospinal and reticulospinal tracts responsible for modulating the activity of commissural and ipsilateral interneuronal populations of the spinal cord during precise stepping represent the biological basis for producing optimal task-space solutions producing the proper locomotor output required by the environment. Data combined from studies by Mitchell et al. (2016) and Ueno et al. (2018). Arrow heads excitatory input; Bullets - inhibitory input. 


\subsubsection{Assessment of gait deficit after stroke}

Most neurological causes locomotor asymmetries owing to the damage of unilateral or bilateral centers responsible for governing locomotion. Unilateral stroke, for example, causes hemiparesis, which is characterized by one sided debility of the body, which may result in bone and muscle frailty. This debility is associated with unilateral gross motor deficits characterized by impaired spatiotemporal activation of muscular synergies, which results in an impairment of multisegmented dynamics necessary for optimal motor output. Ultimately, these deficits induce asymmetric gait deficits characteristic of hemiparetic limping. The asymmetry of hemiplegic gait is produced by an asymmetric spatiotemporal muscle activation most significantly manifested in the shortening of the extensor-associated stance phase and the lengthening of the flexorassociated swing phase of the step cycle on the hemiparetic side. This trend has not yet been explored across a range of locomotor speeds in healthy or hemiparetic animals, which is essential for the purpose of locomotor output.

Post-stroke morbidity in the surviving population includes gross motor impairments that pose a challenge for quantitative evaluation in both post stroke patients and animal models of neurologic impairment. In the clinical setting, these motor impairments are measured using subjective criteria which are more sensitive to severe rather than moderate impairment exhibited by most patients. Similarly, such subjective assessments of post-injury motor behavior in animals are common, e.g., the Basso, Beattie, and Bresnahan (BBB) locomotor scale method ${ }^{38,39}$. While these subjective evaluation methods help translation between gait rehabilitation studies in quadruped animal models and humans, motor deficits associated with separate muscle groups are not addressed. Moreover, the assessment of motor cortical contribution to locomotion, as the putative culprit of motor deficit in cerebrovascular accidents, can only be obtained indirectly even using the most novel automated quantitative methods. These methods rely on open-field or linear walking tasks, and may only be used for gross motor evaluation, giving little regard to the 
synergistic deficits associated with locomotor impairment ${ }^{40}$. These tasks do not require cortical contribution and can be performed by the neural mechanisms of the spinal cord, which has been known since the Brown studies in decerebrate animals at the beginning of the $20^{\text {th }}$ century ${ }^{41,42}$. As such, the central pattern generator (CPG) network located in the spinal cord is spared in most animal models of cerebral damage, such as the case in the middle cerebral artery occlusion model. Essential cortical contribution to these spinal mechanisms has been experimentally implicated in tasks that require anticipated postural adjustments and reaching ${ }^{43}$, as well as precise stepping ${ }^{44}$, which raises the demand for development of new gait assessment practices.

Other means to assess motor function after neurological injury involve the investigation of muscular synergies using surface electromyographic recordings ${ }^{20,45-47}$. These methods require technical skill which involves mathematical computation and decomposition of EMG data, which proves sufficient for the evaluation of synergistic impairments in post-stroke patients, but is of little use in the experimental setting, where the most common models are the rodent models. For that purpose, acute transcutaneous recordings of EMG activity have proven to be sufficient for the examination of motor impairments in animal studies, where researches use similar mathematical decomposition methods to those of evaluating data from patient studies ${ }^{23}$. These studies, however, employ these highly sensitive tools only in isolated settings, where the data is obtained from restrained anesthetized subjects with little regard for the various degrees of freedom associated with in vivo locomotion.

\subsubsection{Assessment of gait deficits after stroke in rodent tMCAO models}

For the purpose of investigating in vivo locomotion post neurological injury, several studies have employed chronic electromyographic recordings from cats and primates ${ }^{48-50}$. These models provide some convenience, as cats and primates are comparatively larger animals (as compared to rodents), which eases surgical approaches as well as recovery after the surgical procedure. Only recently, more novel commercial systems have been made available which aim to 
investigate unrestrained animal behavior in vivo, but they are restricted to relatively insensitive single-channel EMG recordings ${ }^{51}$, use few one-dimensional datasets and are used quite rarely ${ }^{52,53}$, which makes them unsuitable for the investigation of muscular synergies after unilateral brain injury. This raises the need for quantitative and qualitative electromyographic studies in rodents which can be applied in vivo. For the purpose of investigating corticospinal regulation of asymmetric gait, novel asymmetric gait quantification tasks may be used in conjunction with such electromyographic approaches ${ }^{54,55}$. This can aid the development of telemetric analysis, as the identification of synergistic function can be reduced to simple analyses using low-cost commercially available systems.

\subsubsection{Gait deficit assessment aids the investigation of impediments of the motor hierarchy}

The purpose of creating novel behavioral tasks ultimately does not only serve the purpose of diagnosing and quantifying locomotor deficits. The motor output, in essence, represents the output of complex neurological processes which navigate complex task space in order to produce the optimized motor behavior clinicians define as proper motor function. The nature of movement in multisegmented organisms, ranging from arthropods to higher primates and humans, is to perform movements of the simplest possible choreography with the purpose of minimizing energetic utilization. As such, from an engineering standpoint, the reaching as well as locomotor behavior of complex multisegmented organisms represents a finely-tuned (elsewhere suboptimally tuned control system) which relies on certain dynamic characteristics preserved for minimal inertial expenditure. These (sub)optimal segmental dynamics define the output of such a control system, or rather, they define the output of the state-space of numerous complex parameters. In that sense, if one can define, or mathematically model, the state-space output of the multisegmented dynamics, then perhaps it is possible to evaluate the extent of the deficit from output impairments alone. Such approaches tend to focus on understanding the gravitational, 
inertial as well as muscle synergistic behavior alterations following neurological injury. Likewise, these approaches can be used to evaluate the deficits of defined architectures which are able to produce optimal as well as suboptimal behaviors (or capture) of both proper as well as impaired motor function.

\subsection{The Spinal Central Pattern Generator in Development}

\subsubsection{Development of the spinal interneuron population is tied to retinol signaling}

During spinal cord development, as is the case in other parts of the central nervous system, retinol signaling and retinol converting enzymes cooperate with Shh-independent signaling of interneuronal neurogenesis (Figure 4C) ${ }^{56,57}$, which exhibits a dynamic oscillatory profile through downstream transcriptional targets ${ }^{58}$. Though the availability of retinol is essential to the induction of gene targets necessary for development, the essential storage tissue is not located in the spinal cord, but in the liver, which raises a transport demand for this hydrophobic compound. Specifically, retinol-binding protein 4, a retinoic-acid homeostasis system component involved in peripheral transport of retinol to retinol-converting tissue ${ }^{59}$, is upregulated in the liver and pancreas during mouse embryogenesis ${ }^{60}$, and reaches zenith expression during the Theiler Stage 26, 16 to 17 days after conception in mouse embryogenesis, which are associated with terminal maturation of the rodent hindbrain and spinal $\operatorname{cord}^{60}$.

The mechanism of RA-dependent induction of transcription has been well documented. As a transcriptional co-activator, RA binds the heterodimeric retinoic acid receptors RAR and RXR to recruit histone acetyltransferase ${ }^{66}$, which facilitates expression of the promoter in the vicinity of the retinoic acid response cis-element (RARE) genes. In the unligated form, the RAR-RXR heterodimer is preferentially localized in the cytoplasm, though the remaining nuclear heterodimers recruit histone deacetylases (HDACs) and downregulate the expression of RARE genes $^{66,67}$. Upon induction of transcription, a time-dependent chromatin decondensation spanning 
from the $3^{\prime}$ locus of the Hox gene cluster is associated with a latent anterior-posterior expression of b1 through b13 gene loci in the same order as their $3^{\prime}-5^{\prime}$ location on the locus ${ }^{68}$. The long-range transcriptional enhancement is further dependent on the cooperative behavior of multiple RAREs ${ }^{69}$.

Interestingly, the transcription activation mechanism of the Hox cluster seems to be dependent on nuclear $\beta$-actin polymerization which cooperates with the embryonic autoregulated RNAPIIrecruiting Prep1 and $P b \times 1$ circuit $^{79}$. Microarray analysis of the developing rat hindbrains revealed several transcription factors such as Onecut1 (HNF-6), Meis2, mafb2, Hoxa2, Mab21, Mafb, Etv4, Zpfm2 and Znf503 whose expression plateaus during the postnatal days of development and before the onset of hearing in the development of hindbrain ${ }^{80}$, which are crucial elements for the development of the central nervous system and spinal cord in general. The expression of Mafb(also known as kreisler) ${ }^{63}$, a transcription factor known to be involved in the process of interpreting pathfinder cues in the hindbrain ${ }^{81}$, extraocular muscle innervation ${ }^{82}$ and Renshaw cell development ${ }^{83}$, is also known to be RARE promoted element, and is completely obliterated from tissue developed in retinoic acid deprived growth conditions. Mafb is also a marker of V1 interneurons ${ }^{84}$.

Znf503Nolz1 is also reported to bear a 5' retinoic-acid promoter element upstream of the gene for this striatum-enriched zinc-finger transcription factor. This gene has been correlated to neural differentiation at P19 in rats ${ }^{87}$. In a different mouse models of hindbrain maturation, Hoxb1/2 regulates the expression of Rig ${ }^{88}$. Furthermore, early Hoxa1, Krox20 and Mafb (kreisler) mediated segmentation of rhombomeres $r 4 / 5, r 3$ and $r 5$, and $r 5 / 6$, respectively, is grossly affected by Raldh2 ${ }^{-1-89}$. Furthermore, Onecut is an important transcription factor necessary for dorsal interneuron development ${ }^{90}$, the Pbx family of genes is essential for normal locomotion ${ }^{91}$, and Iroquoix genes (Irx 1-6) are necessary for spinal cord neurogenesis ${ }^{92}$. In other studies, Nolz1, a Noc-Elbow-TIp-1 (NET) family member, is induced by the retinol signaling cascade and specifies 
the fate of motor neurons originating in the lateral motor column, and become destined towards innervating limb muscles roughly around the Hamburger-Hamilton 21-23 stage of chick embryo development ${ }^{93}$, which corresponds to the timing of development of distinct limb buds ${ }^{94}$, around Stage 20 of Carnegian development.

\subsubsection{Spinal interneurons have a distinct developmental phenotype}

Spinal motor interneurons and motor neurons are generally thought to arise from five distinct progenitor centers located centrally in the thoracic and lumbar enlargements of the spinal cord (Figure 4A and 4B). Termed $\mathrm{p} 0$ through $\mathrm{p} 3$, they represent dorsoventral distribution, with centers numerated with 0 being located most dorsal, and 3 located most ventral. The progenitor cell location giving rise to the $\alpha$-motor neuron, termed pMN, lies between the positions of the $\mathrm{p} 2$ and p3 progenitor centers. The designation of the mature interneurons keeps relatively the same profile of position-related number designations, with the exception that the $\mathrm{p} 0$ progenitor cells give rise to V0 interneurons, which are segregate most ventrally in lamina IX, and send commissural projections spanning one to four segments. The $\mathrm{V} 1$ and $\mathrm{V} 2$ originate from the $\mathrm{p} 1$ and $\mathrm{p} 2$ progenitor centers, and represent a set of ipsilaterally projecting interneurons, with $\mathrm{V} 1$ innervating a-motor neurons within the same segment, and V2 innervating motor neurons and interneurons within one to four segments. The V3 interneurons originate from the p3 progenitor centers, which migrate relatively little and project commissural inputs to lamina IX within the same segment ${ }^{1}$.

The commissural interneuron V0 population is separated into two subtypes. V0v represents an excitatory interneuron class positive for Dbx1 (developing brain homeobox 1) and Evx1 (evenskipped homeobox 1), which uses glutamate as a neurotransmitter ${ }^{56,95}$. Similarly, V0d are positive for Dbx1, but negative for Evx1, which has been used for their molecular identification in developmental and functional studies ${ }^{96}$, and are GABA-ergic and glycinergic in their mode of neurotransmission. The V1 ipsilaterally projecting interneurons are positive for En1 (Engrailed 1) and are inhibitory as well, using GABA and glycine as neurotransmitters, and may be associated 
with locomotor speed ${ }^{97}$. Corresponding to their innervation profile, ipsilaterally projecting $\mathrm{V} 1$ and a subset of the V2 interneurons, termed V2b and marked by Gata2/3, are necessary for rhythmic flexor-extensor motor neuron alteration, and bear an inhibitory function ${ }^{98,99}$. Likewise, the V2a subpopulation, marked by $\mathrm{Chx} 10$ (ceh-10 homeodomain-containing homolog 1), of ipsilaterally projecting interneurons have been found to be necessary for modulation of alternation at different locomotor speeds ${ }^{34}$, as well as coordinating precise motor functions during the reaching behavior ${ }^{100}$. The V3 excitatory interneurons, marked by Sim1 (single-minded homolog 1) share two separate innervation fates, with $85 \%$ being commissural, and a smaller subset of approximately $15 \%$ being ipsilateral. A more elusive interneuronal subtype, termed the Renshaw cell was identified as early as the Renshaw and Eccles studies in the 1940s was found to exhibit antidromic inhibition of ipsilateral $\alpha$-motor neurons ${ }^{69,101-103}$. This subtype has a complex pattern of transcription factor expression ${ }^{83}$, which further corroborates that tightly regulated genetic regulation interplays are involved in the development and maintenance of spinal interneuron centers $^{98,104}$. Finally, the pMN population gives rise to two distinct neuronal subtypes - the classical cholinergic a-motor neurons, and the ipsilaterally projecting glutamatergic $\mathrm{Hb} 9$ interneurons. Both cell types express the Hb9/MNR2 transcription factor (for hybrid 9/motoneuron 2 ), which is conserved across the animal kingdom ${ }^{105}$. These interneurons are not classically rhythmogenic, but may modulate the central pattern formation during locomotion, as shown in experiments on fictive locomotion in the rodent spinal cord ${ }^{106}$. 
A.

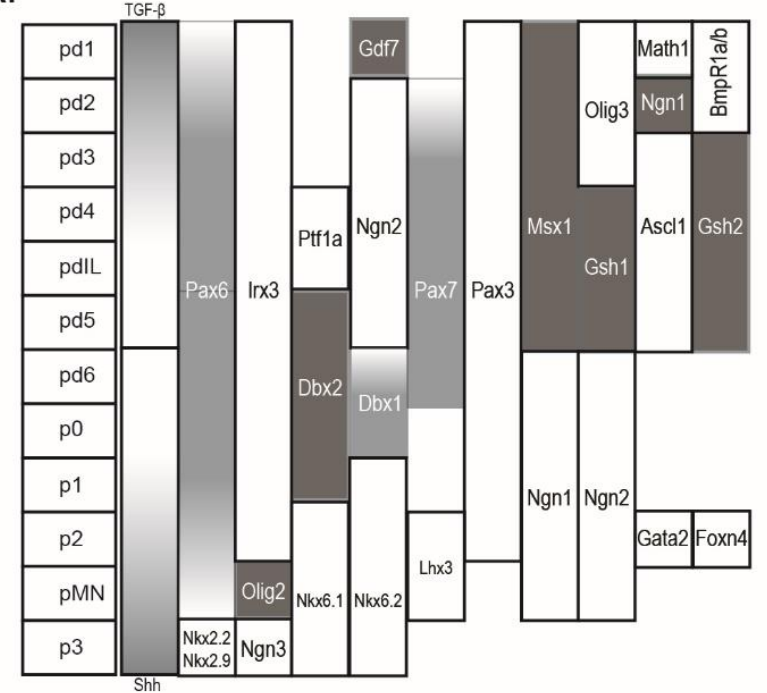

C.

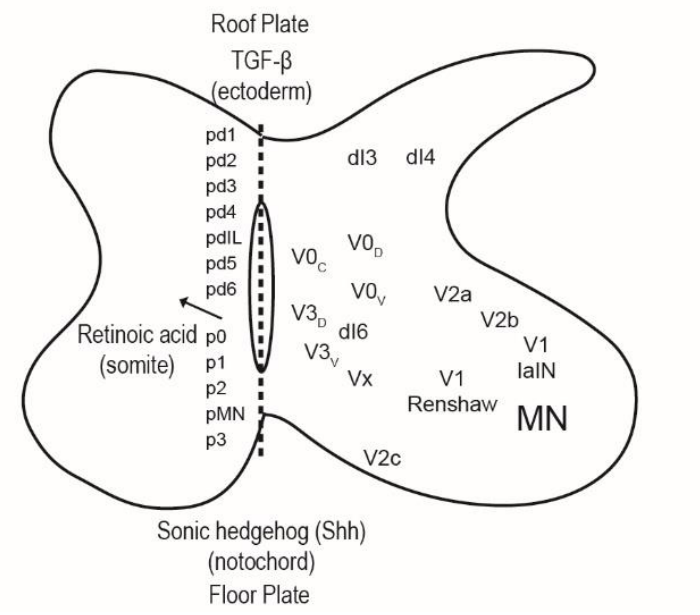

B.

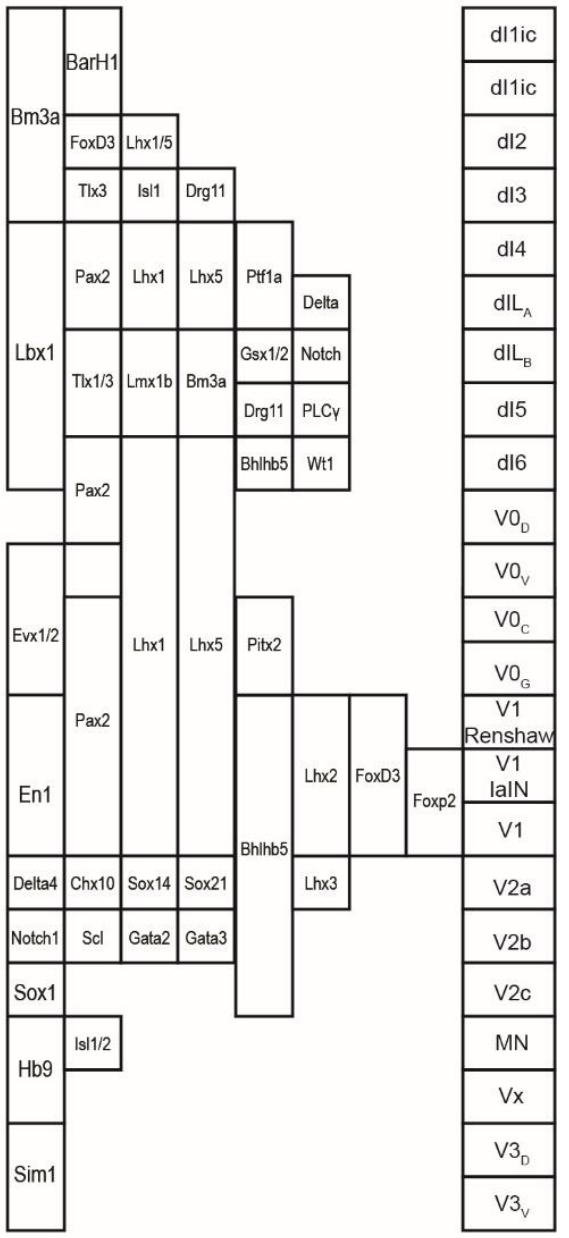

Figure 4. Summary of spinal cord motor neuronal and interneuronal development. A. While in the progenitor zone, neural progenitor cells are in their mitotic phase and exhibit sequential activation of specific transcription factors under ectodermal and notochord derived transcriptional pressures. B. Once neural progenitor cells from the various mitotic zones enter their postmitotic phase, they initiate migratory patterns and start to express various transcription factors unique to their ultimate positional and innervational fate. C. During somite development (inset, left), three major transcriptional pressures take place. TGF- $\beta$ signaling is derived from the ectoderm and is most prominent along the dorsal progenitor zones. The notochord derived Shh signaling is most prominent along the ventral progenitor zones. Finally, tissuederived retinoic acid is produced by various retinol converting enzymes and cooperates with transcriptional signaling from the ectoderm and notochord to drive the various gradients required for terminal motor neuronal and interneuronal differentiation (inset, right). Adapted from William A. Alaynick et al. (2011) and William A. Alaynick et al. (2015).

It is important to note that while some transcription factors are distinctly related to certain spinal interneuron types, which makes them ideal targets for transcription factor-targeted genetic ablation, these are not the only transcription factors involved in their development. For example, 
while still in the progenitor zone, the progenitor subtypes begin to express a different array of transcription factors under the ectodermic pressure by TGF- $\beta$. This pressure drives the initial expression of several bone morphogenic protein types (BMP 4-7), as well as Gdf7 and Wnt ${ }^{107}$. This pressure is distributed in a dorsoventral pattern, whereby the largest TGF- $\beta$ and BMP pressures are distributed along the dorsal portion of the developing somite, which ultimately gives rise to the dorsal progenitor zones 1 through 6 . During early BMP signaling, three neighboring domains induced by basic helix-loop-helix transcription factor expression patterns are formed along the extreme dorsum and ventral to the roof plate of the developing somite. The predominant transcription factors involved here are Math1, Neurogenin 1 and 2 (Ngn1 and Ngn2), and Mash1 ${ }^{108}$, which regulate the further downstream expression of other transcription factors necessary for the generation of the distinct interconnectivity subtypes. Likewise, the development of the progenitor zones for motor neurons and interneurons, is under signaling control by sonic hedgehog (Shh) which is derived from the notochord, and is also distributed along the dorsal ventral axis, but this time with the largest signaling pressure being present on the ventral side of the developing somite. There are two distinct modes of signaling that regulate the development of the ventral neuronal subpopulations, termed Shh-dependent and Shh-independent. The differential signaling by these transcription factors is further supplemented by retinoic acid produced by retinol converting enzymes, and is intrinsic to the somite.

It is important to keep in mind that the Nkx class transcription factors and Olig2 are induced directly by Shh signaling, termed Class I - Shh induced, while the Pax family transcription factors, the Dbx transcription factor family, as well as Irx3 are repressed by Shh, which is characteristic of their dorsoventral distribution, and are termed Class II - Shh repressed. By the same token, TGF- $\beta$ induced transcription factors, Olig3, Math1, Ngn1, BmpR1a/b and Gdf7 are distributed along the roof plate, termed Class A - TGF- $\beta$ dependent ${ }^{109,110}$. The Class B - TGF- $\beta$ independent transcription factors are generally distributed medially in the developing somite and are 
represented by Ptf1a, Gsh1/2 and Ascl1. It is sometimes common to identify the distinct neuronal subpopulations along the class of transcription factors they end up using - Class I, Class II, Class $A$ and Class B neurons.

For locomotion, the ventral groups of interneurons represent the more interesting group for research. During the mitotic phase of development, this array of initial signaling factors induces the expression of Pax6 and Irx3, which are distributed most notably between both ventral and dorsal progenitor zones, with the exception of the p3 zone, which expresses Nkx2.2 and Nkx2.9. This progenitor zone maintains a more unique sequence of transcriptional cascades than the other progenitor zones, as it also remains positive for Nkx6.1 and Nkx6.2 throughout the mitotic phase $^{111}$. Like the p3 zone, the p2 zone expresses Nkx6.1 and Nkx6.2, but is also characterized by the presence of Lhx3, Ngn1, Ngn2, Gata2 and Foxn4. The p1 zone also shows positivity for Nkx6.2 (but not Nkx6.1), Pax3, Ngn1 and Ngn2. The p0 zone is likewise characterized by Dbx1, Dbx2, Pax3, Ngn1 and Ngn2. Lastly, the pMN zone shows little staining for Pax6, expresses Olig2, Nkx6.1 and Nkx6.2, Lhx3, Ngn1 and Ngn2 ${ }^{112-114}$.

Another recently identified transcription factor $\mathrm{Pbx3c}$, a Pbx3 subtype, has been identified to play a key role in the development of the interneuronal populations relevant to locomotion. $\mathrm{Pbx} 3$ is a TALE (three amino acid loop extension) type $\operatorname{protein}^{72}$ which bears homology to $\mathrm{Pbx} 1$ - a protein implicated in pre-B cell leukemia ${ }^{115}$ whose knockout impairs the central pattern generator for respiration ${ }^{116}$. This transcription factor is expressed predominately in glutamatergic spinal interneurons as early as e10.5 and produces significant locomotor deficits (Catherine Anne-Marie Rottkamp, 2008).

\subsubsection{Dorsal interneurons mediate somatosensory regulation of locomotion}

Historically, a population of interneurons which has a dorsal progenitor location in the developing spinal cord, termed dl (for dorsal interneuron) has been classically thought of having solely a 
somatosensory relay function ${ }^{112}$. These interneurons originate from progenitor domains $\mathrm{pd} 1-6$ and pdIL (located dorsally between $\mathrm{pd} 4$ and pd5 progenitor locations). dl1 through dl3 are thought to represent glutamatergic somatosensory relay interneurons with innervation targets at higher brain centers. dl4 interneurons represent ipsilaterally projecting somatosensory associative inputs which GABA-ergically modulate cutaneous and proprioceptive signals. dl $\mathrm{L}_{A}$ and $\mathrm{dl} \mathrm{L}_{B}$ (for late developing type $A$ and B) are GABA-ergic and glutamatergic interneurons, respectively, which project to dorsal horn interneurons which mediate somatosensory associative inputs. These interneurons, though they may have a modulatory role in central pattern formation for the purpose of locomotion or reaching, they do not represent a part of the classically-viewed forward modes of motor control, and have been largely excluded from modeling investigations.

Some of these more novel finds add new members to the repertoire of locomotion-related interneurons. For example, a set of dorsally derived DMRT3-marked interneurons ${ }^{90}$, termed dl6, mediates locomotion developed in part by Pax3/7 signaling ${ }^{118,119}$. Interestingly, a DMRT3 nonsense mutation is associated with the ability of Icelandic horses to exhibit a pacing gait. Another set of interneurons are the Shox2 positive interneurons, which represent a set of V2a excitatory interneurons specifically related to left-right coordination during walking in rodents.

During placental development, the generation of dorsal interneurons takes place at slightly different timelines. For example, the early phase of dorsal interneuron development takes place between embryonic days 10.5 and 12 (e10.5-e12), and is tied to the generation of sensory interneurons within the dorsal horn. Conversely, e12-e13.5 is the period of development which gives rise to the neurons of the marginal layers (laminae I through IV), and is majorly related to the maturation of $\mathrm{dlL}_{A}$ and $\mathrm{dlL}_{B}$ interneurons ${ }^{117}$. The ventral interneurons are generated along similar timelines. For example, la inhibitory interneurons, as well as V1 and Renshaw cell interneurons are derived as early as e9.5 $5^{84}$. The V0-V3 interneurons, as well as the ventral motor neurons can be distinguished as early as $\mathrm{e} 10$, and reach their peak migratory potential around 
e13. Most of the migratory post-mitotic patterns take place between e13 and e16. By e17 and throughout the postnatal days of development, these subpopulations have matured and reach their final destinations within their respective laminae ${ }^{120}$. In the rodent model of spinal interneuron neurogenesis, it has been demonstrated that the development of the flexor and extensor interneurons exhibits time segregation, with the neurons projecting on motor neurons innervating the tibialis anterior muscles efferents developing predominately between embryonic development days 9 and 12 (e9-12), while the development of the interneurons innervating the extensor gastrocnemius motor neurons develop predominately between embryonic days e11 and e13. Furthermore, the somata of the extensor and flexor related lower motor neurons reside in a spatially segregated manner, with a distinct medio-lateral segregation in the dorsal horn ${ }^{121}$.

\subsubsection{Interneuronal populations in charge of central pattern generation}

The spinal cord's intrinsic property to generate alteration of stance and swing related muscular activity has been known since the investigations of Sir Charles Scott Sherrington at the beginning of the $20^{\text {th }}$ century ${ }^{122}$, and the later investigations of Thomas Graham Brown further demonstrated that the spinal cord has a further function in regulating intralimb coordination through putative interneuronal networks which reside in the spinal cord, and that these networks are sufficient to produce contralateral reflex coordination for generation of various types of gait ${ }^{41,123}$.

Since, the reflex coordination networks have been investigated in several laboratories, most notably by the investigations by JC Eccles on the nature of the inhibitory pathways from la and Ib afferents, as well as the function of the Renshaw cell ${ }^{102}$, as well as the VJ Wilson lab studies on the excitatory and inhibitory latencies of the spinal monosynaptic reflex systems ${ }^{124}$. Within the same decade, studies by the Lundberg laboratory demonstrated that there are multiple neurochemical means of modulating interneuronal function, such as through dopaminergic neurotransmission ${ }^{125}$. Within two decades, intrinsic spinal locomotor patterns were further 
investigated in the crayfish and lamprey, demonstrating similar interconnectivity modes preserved between lower and higher vertebrates ${ }^{126-128}$.

With the advent of genetic tools for the investigation of molecular biology in neuroscience, it has been demonstrated that the interneuronal populations of the spinal cord mediating the rhythmicity of locomotion are not necessarily of the same developmental phenotype, regardless of their excitatory or inhibitory function. Some of these postulates are key to understanding the functional complexity that these central pattern generator architectures are capable of producing under various modes of driving, which is exemplified by these networks' ability to produce varying behaviors $^{129}$.

The corticospinal pathways, however, exhibit rare direct corticospinal-motoneuronal innervation. While this direct CS- $\alpha M N$ connectivity is associated with manual dexterity and is greatest among old-world primates and humans, the more common manner of innervation is corticospinalinterneuronal. Some interesting studies have been performed since the time of JC Eccles using retrograde transsynaptic labeling, which reveal that the motor cortex innervates distinct interneuronal subtypes, which ultimately represents the major mode of regulating optimal firing of these interneuronal populations for the purpose of locomotor behavior.

\subsubsection{Rehabilitative strategies seek to retrain the spinal centers of pattern formation}

It stands to reason that a moderate ischemic insult to higher centers in the motor hierarchy produces direct damage to the corticospinal tract as well as basal ganglia. As such, these insults spare the lower, spinal, centers from direct injury. Yet, despite the centrality of the injury, there is a marked maladaptation of locomotor function, which results in permanent alterations of the affected motor actuators, resulting in detriments to synergistic muscle activity as well as gross

impairments to the musculoskeletal system on the hemiplegic side ${ }^{24}$. Historically early 
interventions have been thought to be most effective. These rehabilitative interventions often seek to retrain the lower centers of pattern generation with the purpose of restoring function ${ }^{22}$. As little regard has been given to the exact nature of deficits induced by central injury, modeling studies that link damage to the descending tracts in conjunction with other pathophysiological markers of hemiparesis are of great interest.

\subsection{The Motor Unit}

Motor control across the animal kingdom is tasked with adapting the position of an organism's body in three dimensional space, and in relation to objects or other organisms. In all organisms, motor control is adapted to the type of movement an organism is capable of performing. Mammalian motor control, for example, has the challenge of manipulating multisegmented bodies with the aid of contractile spring-like muscles, which attach to bones through non-contractile segments called tendons. The intersegmental, inertial, and gravitational interactions of these segments in three dimensional space constitute the forward musculoskeletal dynamics, which a controller located in the central nervous system must take into account in order to produce coordinated movement.

As the contractile muscles are the main voluntary force producers influencing segmental geometry, they need to be driven in a coordinated fashion in order to refine movement and adapt

to changing circumstances. The main controller for musculoskeletal interaction is represented in the central nervous system. For this purpose, an $\alpha$-motor neuron innervates several homonymous muscle fibers to constitute a motor unit. This concept was first introduced by Sir Charles Scott Sherrington in $1925^{130,131}$.

For a typical muscle, anywhere between 10,000 and over 1,000,000 muscle fibers are innervated by 100 to 5000 a-motor neurons. The ratio of muscle fibers innervated by a single $\alpha$-motor axon is termed the innervation number. This number ranges from a very low, about $5-10$ for rectus 
lateralis or tensor tympani in the fine motor control pertinent to the sensitivity of specialized senses, all the way to 2000 for the significantly larger gastrocnemius.

In response to action potentials originating in the $\alpha$-motor neurons, muscles exhibit twitch contraction. This behavior differs in different types of muscle fiber and is classified according to the contraction speed, peak force and fatigability. The time necessary for a muscle fiber to achieve maximum contractile force is termed the contraction time of that motor unit, which is further distinguished into fast and slow twitching. Most refined voluntary contraction of muscles is the result of summation of these tetani, and maximum force is achieved by increased frequency of firing in the $\alpha$-motor neurons.

Furthermore, the contractile properties of motor units are distributed between long twitches and small forces to short twitches and high forces. In humans and other mammals, the majority of motor units show intermediate contraction times of $40 \mathrm{~ms}$, with a range of $20-80 \mathrm{~ms}$, and most commonly produce intermediate forces. Muscle fibers can be distinguished morphologically by histochemical staining for adenosine triphosphatase. On that basis, the slowly contracting type I fibers can be distinguished from the quickly contracting type II muscle fibers. Further, the type II fibers are separated into the Ila fiber types and Ilb, Ilx or Ild fiber types, with Ila fibers being most oxidative and least fatigable, and $\mathrm{ll} / \mathrm{d} / \mathrm{x}$ being least fatigable. Another phenotypic distinction of these fibers is the myosin heavy chain isoform expressed. Type I fibers express myosin heavy chain-I, while type II fibers express predominately myosin heavy chain-Ila, and myosin heavy chain-Ilb/Ix, named conveniently for the different muscle fibers in which they were identified ${ }^{132-}$ 134 .

The order of recruitment of motor units during the process of contraction also has a particular form. If we consider the spectrum of fatigability and force output, lightly forceful, slowly contracting, and non-fatigable motor units are the first ones to become recruited during a forceful contraction. Inversely, the last units to become recruited are those with short contraction times, 
high forces, and high fatigability. Muscle contraction is also regulated by a recruitment and discharge profile of the motor units and the motor neurons that drive their contraction. This process is largely accommodated in spinal networks and is not controlled voluntarily. The recruitment function in the spinal cord is distributed across several parameters of the motor units, such as the size of the motor neuron, the axonal diameter and conducting velocity, as well as the force and fatigability of fiber that the motor neuron innervates ${ }^{130}$.

Yet, some simple relationships can be inferred from the size of the motor neuron. For example, the motor neurons with a smaller surface area have a higher resistance, and as a result, excitatory postsynaptic potentials (EPSPs) generate action potentials sooner than in a lower-resistance large motor neuron. Any size motor neuron is linearly recruited as a function of the presynaptic firing rate, unless this firing rate is modified in some way by monoaminergic input from brain stem or other inhibitory interneurons. In this scenario, the firing rate of the post-synaptic cell can undergo up to a ten-fold enhancement of firing rate under linear excitatory input. This enhancement can sustain a higher discharge rate in a motor unit despite the initial depolarizing input. This property is termed self-sustained firing. Naturally, larger motor units, wherein a single axon terminates on a large number of muscle fibers, tend to be more forceful, less precise and tend to be recruited later than small motor units during the recruitment phase of contraction under increasing force demand. This principle is termed the Henneman's size principle.

\subsubsection{The aging process affects the motor unit}

Skeletal muscle does not exist in isolation, but rather requires a healthy interaction with the central nervous system for healthy maintenance. In aging, this relationship is often impaired to varying degrees, and it is important to understand the processes that underwrite fatigue, and their alteration in aging. The neuromuscular interaction at its unit level is termed the motor unit, and pertains to the a-motor neuron and the muscle fibers that it innervates. The contraction rate of the muscle fiber is physically limited by the rate of energetic utility from the splitting of adenosine 
triphosphate, which is, in turn, conferred by the catalytic rate of the actomyosin adenosine triphosphatase (ATPase) expressed in the particular muscle fiber. In its simplest parameters, motor units are separated according to size, or number of muscle fibers innervated by a single motor neuron. In senescence, one or more aspects of the neuromuscular architecture are altered, resulting in decreased motor unit size, as well as a decreased capacity for force generation in the aging muscle fiber ${ }^{135}$.

\subsection{The Sirt1/PPARy/PGC1a Axis in Neurological Injury-Related Muscle Damage}

The Sirt1/PPARy/PGC1 $\alpha$ signaling axis, whose activity has been implicated in the development of the neuromuscular junction program as well, is particularly sensitive to denervation injury of muscle, which further exacerbates the aging phenotype ${ }^{136}$. Neuromuscular junction instability as well as motor neuron death is a common phenotype associated with aging, and it appears that fast-twitching neurons and the overlaying neuromuscular junctions are more vulnerable to the processes of senescence, perhaps due to the comparatively lower capacity of fast glycolytic muscle fibers to buffer oxidative stress. Concurrently, the loss of NMJ stability, fast a-motor neuron death, impaired mitophagy, as well as transcriptional alterations contribute to the denervated or hemiplegic muscle phenotype and generally need to be considered when trying to understand the altered biomechanical properties of aged muscle.

\subsubsection{Sirt1}

Sirtuin 1 belongs to a family of NAD-dependent histone deacetylases conserved from yeast to higher mammals. In yeast, it has been found to contribute to increased cellular lifespan owing to its function of activating a repertoire of genes involved in protection against cellular stress. In mammals, it has been implicated in various modes of regulation of energetic metabolism and DNA-damage response ${ }^{137-140}$. It has been demonstrated previously that in cellular models of DNA damage, Sirt1 becomes ubiquitinated, which is necessary for its function in the DNA damage 
response $^{138}$. Moreover, the relationship between ubiquitination and Sirt1 deacetylase activity seems to be more dynamic than previously thought as well, and Sirt1 loss is associated with defective protein quality control, and aggregation of ubiquitinated species ${ }^{140}$. In order to evaluate the relationship of Sit1 to stroke-related muscle injury, experiments that uncouple any potential impediments of the ameliorative PGC1a mitobiogenesis program from the expression of p65dependent muscle ubiquitin ligases might require further attention. For that purpose, muscle creatine kinase promoter dependent Sirt1 overexpressor line that specifically overexpresses Sirt1 in skeletal muscle ${ }^{141}$ previously reported to relieve atrophy of slow-twitch fibers in a Duchenne Muscular Dystrophy (DMD) mouse model may increase the presence of Sirt1, and enhance PGC1a transcriptional activity. Transient middle cerebral artery occlusion (tMCAO) in this overexpressor model is expected to improve PGC1 $\alpha$ activity and mitochondrial biogenesis and should ameliorate the expression of FoxO3a-dependent atrophy genes (Atrogins), which would decimate ubiquitin ligase upregulation seen in wild-type mice post-stroke. As a deacetylase responsible for the transcriptional activation of many factors and co-factors, Sirt1 tends to associate with these elements in the nucleus and is a known component of the transcription apparatus.

\subsection{2. p53}

p53 has initially been identified as a $53 \mathrm{kDa}^{142}$ tumor suppressor protein involved in cell-cycle arrest in the G1 phase of mitosis upon DNA damage ${ }^{143}$, and has since been investigated as a target for anticancer therapy ${ }^{144}$. This protein binds its DNA targets as a tetramer ${ }^{145-148}$, which is facilitated by p53 acetylation ${ }^{149-151}$. Other studies have revealed the complexity of p53 biology ${ }^{152}$, which is not limited only to acetylation status, but also to nuclear import, ubiquitination, neddylation and sumoylation ${ }^{153-156}$. In cellular manipulations, deacetylation of p53 protein is necessary for its ubiquitination, and subsequent degradation ${ }^{157}$ or subcellular localization ${ }^{158}$. 


\subsubsection{PGC1 $1 \alpha$}

PGC1 $\alpha$, or peroxisome proliferator activated receptor gamma co-activator 1 (PPARy co-activator 1a) belongs to a family of transcriptional co-activators which regulate the survival pathway, as well as modulate thermogenic expenditure through the upregulation of uncoupling protein 1 (UCP1). Furthermore, $P G C 1 \alpha$ is a ubiquitous transcription co-facto ${ }^{159}$, which among other functions has been shown to determine oxidative metabolism of skeletal as well as cardiac muscle ${ }^{160,161}$. In other studies, it has been suggested that PGC1a is not absolutely necessary for mitobiogenesis $^{162}$, but it seems evident that it remains indispensable for controlling the content of myosin heavy chain subtypes through hypoxia-inducible factor $2 \alpha(\mathrm{HIF} 2 \alpha)^{163}$. This illustrates that while PGC1a is a useful tool for the determination of fiber type fate, it might not necessarily be the only pathway necessary for the maintenance of fiber type, and other transcriptional pathways may participate in the interplay as well. PGC1a has been found to have an additional role in reversing atrophying damage induced by the FoxO pathway ${ }^{164,165}$.

\subsubsection{FoxO3a}

The Forkhead box (FOX) family of transcription factors represent a set of 39 transcription factors divided into 19 subgroups designated A through S. A member of this family of transcription factors (Daf-16) was initially identified as a nuclear protein crucial for the development of the Drosophila $\operatorname{larvae}^{166,167}$, and later a fusion protein of a homologous domain with Pax3 had been identified in the development of rhabdomyosarcoma ${ }^{167-169}$. Later, Daf-16 had been identified as key regulators of development, apoptosis and longevity in C. elegans ${ }^{170}$, and the FoxO ortholog in mammals was identified to be antagonistically regulated by phosphorylation, whereby the dephosphorylated FoxO3a protein accumulates in the nucleus, and the phosphorylated form interacts with the 143-3 protein to facilitate nuclear shuttling ${ }^{171-175}$. The four members of the FoxO subgroup were later identified for their tumor suppressor capability and cell cycle regulation ${ }^{176,177}$. More recently, an evolutionary study has demonstrated that acquisition of cysteines in the FoxO3 and FoxO4 
transcription factors enables them to form homodimers and heterodimers with each other, as well as heterodimers with nuclear importers Importin-7 and Importin-8 (IPO-7/8), which enables FoxO nuclear translocation under oxidative stress ${ }^{178}$.

For the purpose of regulation of striated muscle, it is important to note that both FoxO1 and FoxO3 have been implicated in the regulation of the levels of atrophy markers MuRF-1 and Atrogin-1, both in cultured myotubes, as well as cardiomyocytes ${ }^{179,180}$. Moreover, FoxOs have been implicated in the regulation of skeletal muscle atrophy under conditions of starvation, muscle denervation and oxidative stress ${ }^{181-184}$. Naturally, further post-translational modifications, such as deacetylation by $\mathrm{Sirt}^{158}$, regulate the fate of the FoxO proteins, and regulate their transcriptional activation.

\subsubsection{MuRF-1}

Muscle-specific RING finger-1, a member of the RING B-box, coiled-coil superfamily of E3 ubiquitin ligases ${ }^{185}$ has the specific role of maintenance of the thick filament structure ${ }^{186}$ through heavy-chain protein ${ }^{187,188}$, and is specifically associated with type II muscle fibers. The ligase has also been termed striated muscle-specific tripartite motif 64 (TRIM64) ${ }^{189}$. It has been identified as one of the key actors in varying types of muscle atrophy not restricted to denervation, fasting, glucocorticoid induced muscle wasting, immobilization induced muscle atrophy, hemodialysis, the

hindlimb suspension model as well as microgravity induced muscle atrophy ${ }^{187,190-194}$. This ubiquitin ligase is specifically involved in maintenance of striated muscle during adulthood ${ }^{195}$, as opposed to the MuRF-2 (TRIM55) and MuRF-3 (TRIM54) isoforms which are preferentially expressed during neonatal development. The MuRF-1 isoform is composed of a RING domain necessary for $\mathrm{E} 2$ ubiquitin ligase interaction at the $\mathrm{N}$-terminus of the protein ${ }^{196}$, a MuRF family conserved domain found in the other two isoforms, a B-box zinc-finger domain in the central region, as well as two coiled-coil domains followed by an acidic tail domain in the C-terminus ${ }^{197}$. Furthermore, it is interesting that the N-terminal RING domain of this ubiquitin ligase may also 
have a transcriptional regulatory role during its interaction with glucocorticoid modulatory element binding protein 1 (GMEB-1) ${ }^{186}$.

\subsubsection{MafBx/Atrogin-1}

MafBx (muscle-specific F-box protein, also termed Atrogin-1) has been identified initially as a muscle-specific E3 ubiquitin ligase upregulated during muscle wasting associated with diabetes, fasting and renal failure ${ }^{198}$. Since, it has also been identified as a key player in denervationinduced loss of muscle mass that lies downstream of the p38-MAPK signaling axis ${ }^{199,200}$, as well as the eukaryotic initiation factor complex 3 (elF-3) ${ }^{201}$. This E3 ligase contains a leucine zipper (LZ) and a leucine-charged residue-rich domain (LCD) in the N-terminal portion, followed by an F-box domain in the central region. These three regions are flanked by two nuclear localization signals (NLS). This structure is followed by a cytochrome $\mathrm{c}$ binding motif as well as a class II PDZ domain at the C-terminal region. Unlike MuRF-1, which has a tight association with the thick filament structure and function, MafBx has a more pleiotropic role in ubiquitinating protein targets and maintenance of muscle mass, not only restricted to cytoskeletal proteins, but also plasma membrane, extracellular matrix cytosolic, nuclear as well as mitochondrial proteins ${ }^{202}$. For example, in the striated muscle of the heart, MafBx targets the FoxO1 and FoxO3a proteins, and activates their transcriptional activity through ubiquitination ${ }^{203}$. Interestingly, FoxO3a targets the MafBx promoter ${ }^{184}$, and FoxO3a is tightly regulated by both phosphorylation and ubiquitination ${ }^{182}$, and this interplay could point to an interesting feed-forward mechanism by which MafBx activity is promotes itself in a feedforward process.

\subsubsection{Sirt1 and p53 participate in a regulatory interplay}

In turn, p53 protein levels influence the alternative splicing of Sirt $1^{204}$, generating ubiquitously expressed $\Delta$ Exon8 isoforms with elusive regulatory function and minimal deacetylase activity. $\mathrm{A}$ second isoform of Sirt1 presently identified is a deacetylase deficient Sirt1 fragment termed the 
Sirt1- $\Delta 2 / 9$ isoform, which does not deacetylate $p 53$, and is thus supportive of its function ${ }^{205}$. The most recent isoform is the Sirt1- $\Delta 2$ band, which is commonly responsible for the two bands commonly obtained by longer electrophoresis times ${ }^{206}$. This isoform is incapable of binding certain transcription factors such as p53, PGC1a, FoxO1, FoxO3a, PPARa and Akt, but is capable of supporting CREB and TIF1 $\beta$ function. Another possible molecular culprit for the increased Sirt1 protein turnover is the p53 repression-mediated pathway. Sirt1 is a known deacetylase of p53, which renders p53 transcriptionally active in cellular experimental models, enabling it to express proteins of the DNA damage response pathway ${ }^{207}$. Experimentally, this pathway is induced by accumulation of reactive oxygen and/or nitrogen species as in denervation ${ }^{200}$. Interestingly, the Sirt1 gene bears with it a p53 repressor element which is active during nutrient abundance ${ }^{208}$, pointing to a complex homeostatic interplay aimed at switching-off nutrient sensitivity in times of cell suicide. This repressor element is also dependent on Hypermethylated in Cancer 1 (HIC1) ${ }^{174}$, so assaying the affinity of $\mathrm{HIC} 1$ to this element could prove interesting. This interaction is dependent on Hsp25209, and renders p53 a target for the proteasome ubiquitin system in its deacetylated state, which allows for the rapid clearance of $p 53^{210}$.

\subsection{Pathophysiology of paretic muscle after stroke}

The ability of a muscle fiber to generate force under a particular rate of stimulation is termed the force-frequency relationship, and bears a sigmoid shape ${ }^{211}$. The specific shape of the sigmoid waveform is generally distinguished between motor units based on the type of myosin heavy chain (MHC), as well as myosin ATPase which are the main determinants of the contractile properties of the motor unit ${ }^{212}$. Yet, motor unit contraction force and the frequency of stimulation can vary due to many factors originating both pre and post-synaptically at the neuromuscular junction terminal. These factors include, but are not limited to the ability of the motor neuron to generate an action potential, sensitivity of the sarcolemma to calcium flux, extracellular calcium availability, availability of contractile protein and inhibition of calcium dynamics at the actomyosin complex 
due to the presence of reactive oxygen and nitrogen species ${ }^{213}$. Nearly all of these factors seem to be affected to a greater or lesser extent in aging ${ }^{214}$, and some of these issues may be expected in stroke as well.

The main difference in fiber type distribution in patients who have suffered from brain ischemia and that of the healthy senescent patients is evident in a remarkable shift to the type Ilb/x phenotype post-stroke, with a pronounced loss of type I slow and type Ila fibers ${ }^{215}$, though there is little knowledge on whether the metabolic profiles of the fiber types remain coupled post-stroke. Consistent with this loss of slow contracting type I fibers, it is expected that the force-frequency sigmoid profile of paretic tibialis anterior (TA) will experience a dramatic decrease in amplitude and peak force will experience a shift to the right in a direct quantitative needle electromyography experiment in vivo and after a transient middle cerebral artery occlusion (MCAO) model in mice. This shift would account for the need to stimulate at higher frequencies experimentally, and in order to induce maximal contraction of the type II fast-fatigable fibers in the same animal after tMCAO, as compared to the same muscle before injury. Moreover, the loss of amplitude will inevitably be affected by the loss of motor unit number, which is a known complication in stroke muscle ${ }^{25}$. Due to muscle spasticity, it is possible that the baseline of force generated by the paretic muscle at rest will be higher than that of the healthy muscle, leading to an overall flattening of the sigmoid curve.

\subsubsection{Muscle atrophy is more pronounced on flexors in clinical observations}

In the clinical evaluation of locomotor deficits following ischemic brain injury, there is a noticeable trend of foot drag associated with dorsiflexor dysfunction ${ }^{216,217}$. This behavior is akin to the inability of an infant to perform toe elevation in the swing act of locomotor progression due to the lack of corticospinal maturation associated with postnatal development, a time during which the functional execution of stance, which appears to be reflexive in nature. This behavior leads to the 
question of whether an ischemic stroke in adulthood bears the affect of functional regression of the neuromotor system, predominately affecting the dorsiflexor muscle population.

\subsection{Mathematical evaluation of neurological deficits after stroke}

Over the last few decades, there have been several attempts to describe the function the motor cortex has in relation to driving of lower motor centers. Most notably, these studies have focused on the firing properties individual motor and premotor layer 5 neurons during reaching, locomotion, as well as complex tasks such as feeding, defending and climbing ${ }^{43,218-221}$. These studies have been performed predominately in old world primates and cats. As the use of primates for the purpose of experimental research is becoming scarcer due to omnipresent ethical issues, there is a great need to develop novel strategies of assessment of neuromotor deficits in rodents, as well as novel strategies to assess neuromotor impediments in patients through the use of telemetry. Mathematical modeling of ischemic impediments has been employed recently as well for the purpose of establishing correlational models of ischemic volume to neurological function, but they either focus more on general clinical pictures of impairment ${ }^{222}$, or they focus on modeling the inflammatory profile associated with the ischemic brain lesion per $s e^{223}$. These studies, however, do not focus on integrating various parameters of muscle function into a holistic predictive model of output. Other studies aim at modeling gait deficits for the purpose of development of prostheses that aid functional recovery noninvasively ${ }^{224}$, which is the desired direction towards rehabilitative cost reduction ${ }^{225}$. This raises several issues that need to be addressed when investigating the mathematical correlates of motor function. Ultimately, holistic

models that can address this question will undoubtedly have a great application potential into other types of motor dysfunction, not just the ones tied to hemiparetic locomotion. Furthermore, such investigations can aid the development of new low-cost tools which can evaluate impediments and monitor recovery remotely, which can aid the great rehabilitative cost associated with ischemic stroke. 


\subsubsection{Holistic modeling investigates disruption of central drives}

Over the last several decades, there have been several attempts to ascribe higher order functions and ascertain various firing probabilities to neuronal cell populations represented in the primary motor cortex $(\mathrm{M} 1)^{226}$. Some more recent publications have used rotational principal component analysis to represent what many thought to be abstract state space defined by repetitive neuronal firing states during like periods of, say, the extensor phase of locomotion or extensor phase of reaching behavior ${ }^{227}$. For example, as locomotion involves repetitive activation of extensor muscle population, it would stand to reason that motor cortical neuronal populations in charge of recruiting the pattern forming layer of the $\alpha$-motor neurons driving the extensor muscle group would become active during or slightly prior to the onset of the extensor phase of locomotion. What flabbergasts most researchers is the fact that the same centers of the motor cortex become active during many other tasks, ones that do not necessarily involve the same muscle groups. Michael Graziano in $2006^{228}$ laid a good possible definition of such a behavior, one he termed Feedback Re-Mapping, whereby feedback from muscles and other spinal layer centers of generation of central patterns dynamically alter the firing states of the motor cortex. This does not fit into the dogma that the pattern forming layers are necessarily located in the spinal cord. Nonetheless, the spinal cord probably does represent a lower form of central pattern generation, as the same central patterns of locomotion can be elicited even in decerebrate preparations, something which has been hypothesized since the Sherrington-Brown dilemma from over a hundred years ago ${ }^{41,122}$. Since the time of Brown's investigations on the tonic drive of the mesencephalic locomotor region, these experiments have been replicated in the lamprey ${ }^{127}$, the rodent neonatal spinal $\operatorname{cord}^{229,230}$, and many studies have investigated the architecture of spinal interneuronal connectivity, as well as dual-mode neurotransmission that governs this alternation ${ }^{35,95}$, which undoubtedly point to the fact that the spinal cord has an intrinsic property of wave propagation ${ }^{231}$ that when fine-tuned by

predictive and reactive tuning may produce the proper locomotor pattern required by the 
environment $^{232}$. These studies seek to produce neuronal models of the spinal central pattern generator, but few studies have focused on the different modes of regulation of these spinal populations as a whole.

For the purpose of holistic modeling of locomotor behavior, for example, there have been several model iterations from the Proczazka lab ${ }^{233}$ to represent a bipedal central pattern generator with reactive and predictive couplings, and another, solely predictive one, by Sergiy Yakovenko, which involves unilateral and bilateral coupling ${ }^{234}$, an elaboration the intrinsic factors as defined by Thomas Graham Brown in 191141, and external leak-in and self-modulating parameters, termed the extrinsic factors. This model has been used to describe the turning behavior by an animal employing different locomotor speeds on either limb.

\subsubsection{A precise foot-placement locomotor task for the evaluation of descending drive function}

For the purpose of investigating the precise stepping, which is known to directly involve the input of the corticospinal neurons originating in the $M 1$ cortex $^{235,236}$, we have designed a device termed the asymmetric walkway ${ }^{55}$, which induces an accurate stepping behavior on stepping platforms of adjustable inter-stride length (Figure 5). This adjustability of stride length can be used to impose symmetric as well as asymmetric locomotion, and can be used to challenge the paretic side in models of brain injury with the purpose of precise assessment of gait lateralization in moderate forms of unilateral brain injury. Primarily, the difference between the locomotion induced by the asymmetric walkway and split-belt treadmill walking, commonly used in patient studies, is the fact that split-belt walking induces walking at different speeds between the left and right limbs. For that purpose, the study presented herein focuses on an expanded bilateral coupling model, whereby the intrinsic connectivity between left and right half-centers is assumed to be unequal, and in order to dissemble the fact that the contralateral corticospinal tracts predominately project to spinal interneuronal populations. 
There have been several problems with optimizing the more novel computerized models, however, mainly in the fact that it is not often easy to define the proper output that such a network needs to produce in order to solve such a complex problem properly. For the purpose of the experiments in this work, several optimization paradigms were modified in order to enhance optimization with the purpose of reaching the desired output. Primarily, these enhancements involve the simplification of the error function comparing the slope of the locomotor phases, while other error factors are added in order to produce the optimal number of steps, and fix the output of the system of differential equations to physiologically-relevant ranges of locomotion. These were done with the purpose of adapting to the locomotor behavior set by the asymmetric walkway.

\begin{tabular}{|c|c|c|}
\hline Condition & $\begin{array}{c}\text { Left ISL } \\
(\mathbf{m m})\end{array}$ & $\begin{array}{c}\text { Right ISL } \\
(\mathbf{m m})\end{array}$ \\
\hline S15 & 75 & 75 \\
\hline L6R9 & 60 & 90 \\
\hline L9R6 & 90 & 60 \\
\hline
\end{tabular}

Table 1. Summary of locomotor conditions. $I S L$ - left interstride length; $r S L$ - right interstride length.
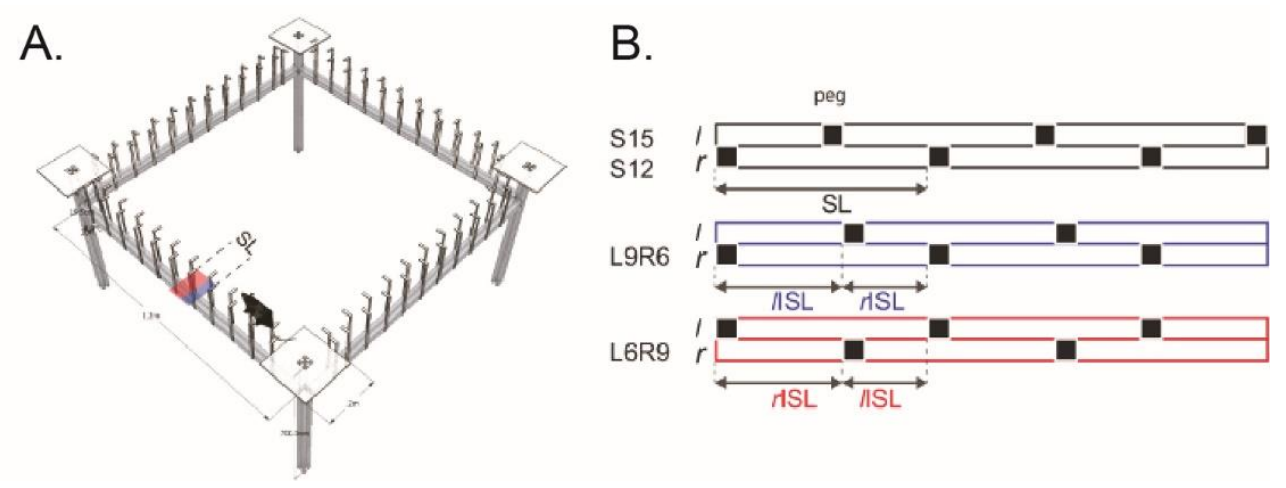

Figure 5. Asymmetric walkway model. A. Schematic of the walkway device used for imposing symmetric and asymmetric gait tasks. B. Peg arrangements setting right ( $r I S L)$ and left (IISL) interstride lengths in relation to the stride length $(S L)$. The four conditions include a symmetrical control locomotor task of a 15 $\mathrm{cm}$ stride length (SL15), a symmetrical task which reduces the stride length by $20 \%$ (SL12), a left-favored gait of a $9 \mathrm{~cm}$ left interstride length and a $6 \mathrm{~cm}$ right interstride length (L9R6) and a right-favored gait of a 6 $\mathrm{cm}$ left interstride length and a $9 \mathrm{~cm}$ right interstride length (L6R9). 


\section{MATERIALS AND METHODS}

\subsection{Animals}

The majority of experiments utilized the rodent model of transient ischemic stroke. For the purpose of biochemical analysis, transient middle cerebral artery occlusion was performed on wild-type C57BL-6 mice, as well as 12 transgenic mice raised on a C57BL-6 background engineered to overexpress native mouse Sirt1 under the promotion of the muscle-restricted creatine kinase MCK (termed MOX), and described elsewhere ${ }^{141}$. For the assessment of gait lateralization, 14 female Sprague Dawley rats (Harlan Laboratories, Indianapolis, IN) were trained to perform a precise stepping locomotor task on shorter interstride lengths $(2-5 \mathrm{~cm})$, which was increased incrementally until the animal properly acquired a walking gait on the symmetric locomotor tasks (S12 and S15), before proceeding to asymmetric locomotor training. Proper walking gait was identified by continuous locomotion, an arched back and exclusion of locomotor behaviors such as running (forelimb and hindlimb coincident swing), galloping (forelimb coincident swing), trot (diagonal forelimb-hindlimb coincident swing) and canter (gallop and trot combination). After a respite period of 24-48 hours, kinematic walking data on the symmetric and asymmetric conditions was acquired using a $60 \mathrm{~Hz}$ High-Definition Handycam HDR-CX380 (SONY, Tokyo, Japan) 8.9MP camera and imported without resampling into a custom MATLABbased software (videoa.m, available as open-source script on the MatlabCentral website, MathWorks, Natick, MA). Stance onset and offset for each limb was marked by foot contact with or departure from the aluminum platform.

All procedures were performed under anesthesia and aseptic conditions, and in accordance with the Institutional Animal Care and Use Committee (IACUC) and Office for Laboratory Animal Welfare (OLAW) at West Virginia University School of Medicine and abides by the National Institutes of Health guidelines for the use of experimental animals. 


\subsection{Asymmetric Walkway}

A low-cost in-house instrument was designed as an open-top plastic box measuring $155 \times 104 \mathrm{~cm}$ and braced with aluminum supports at each corner. Aluminum stepping platforms of dimensions $2.5 \times 1 \times 0.5 \mathrm{~cm}$ were obtained by bending $20 \times 1 \times 0.5$ aluminum pegs at the tip. The stepping platforms were aligned along the perimeter of the box along a grooved brace and secured through screw holes at the bottom of the shaft. Locomotor tasks were produced by changing the distance between pegs at the left and right sides, termed the interstride length (ISL). The symmetric locomotor task with a $15 \mathrm{~cm}$ stride length (SL15) was produced by setting the left interstride length $(I S L)$ and right interstride length $(r S L)$ to the half of stride length of $7.5 \mathrm{~cm}$. The motor system was challenged asymmetrically with an asymmetric locomotor condition induced by shortening the ISL by $20 \%$ and prolonging the $r$ ISL by $20 \%$ to impose a long interstride length $(9 \mathrm{~cm})$ on the right and a short interstride length on the left $(6 \mathrm{~cm})$. A second condition was produced by shortening the right interstride length by $20 \%$ from the symmetric condition to produce a short interstride distance on the right $(6 \mathrm{~cm})$ and a long interstride distance on the left $(9 \mathrm{~cm}$, Table 1 and Figure 5).

\subsection{Middle cerebral artery occlusion - mice}

Anesthesia was induced in a preheated inhalant chamber using 3-5\% isoflurane for 5 minutes. After induction, the animal was transferred to a surgical heat-pad (Kent Scientific, Torrington, CT), lain supine, and dynamically regulated for a body temperature range of $36.5-37.5^{\circ} \mathrm{C}$, and under inhalant $1.5-2.5 \%$ isoflurane delivered via a face-mask system (MouseVent, Kent Scientific, Torrington, CT). A sterile surgical field was maintained throughout the procedure. After verification of anesthetic efficacy by the toe pinch method, the would-be incision site around the neckline was carefully shaved with a thin razor, where an aseptic wash using $10 \%$ povidione iodine was performed subsequently. A single injection of Bupivicaine $\mathrm{HCl}(4 \mathrm{mg} / \mathrm{kg})$ was injected subdermally at the incision site 5 minutes prior to the incision. A $2 \mathrm{~cm}$ incision was performed vertically, and 
approximately $0.25 \mathrm{~cm}$ laterally and parallel to the tracheal line, after which the subdermal panniculus parnosus and connective tissue were blunt dissected to reveal the two lobes of the thyroid gland. The two lobes were separated using blunt forceps, and the right lobe raised past the incision line, and braced using an elastic retractor set to expose a triangular pseudo-space formed by the sternomastoid, the digaster and clavotrapezius muscles. The area was irrigated every 5-10 minutes with warm physiological saline. A final blunt dissection was performed using iris forceps to separate the connective tissue sheathing the triangle muscles, which reveals the common carotid artery (CCA), as well as its two main distal branches - the external carotid (ECA) and the internal carotid artery (ICA). The branches were further blunt dissected from the remainder of the connective tissue, and a 7.0 suture was tied in a Weston knot around the medially running ECA, and abrogating flow through the superior thyroid artery (STA). A second 7.0 suture was placed in a Simple Slider knot around the ICA, and a small arteriotomy was performed using micro-dissecting scissors at the base of the ECA for the Longa, or at the point of the ECA-ICA junction for the Koizumi method ${ }^{16}$. In our experience, the two methods are identical in their induction of the sarcopenic phenotype associated with stroke. A blunted $0.20-0.21 \mathrm{~mm}$ monofilament suture (Doccol Corp., Sharon, MA) with a 0.10-0.15 mm stalk and a 5.0 suture neck was inserted $10 \mathrm{~mm}$ past the proximal ICA knot, and the knot tightened. 60 minutes after onset of occlusion, the monofilament was removed from the ICA lumen to re-perfuse blood-flow, and the ECA stump was ligated by thermocautery. Heart rate was monitored throughout the procedure through a digital monitor (PhysioSuite PS3089B, Kent Scientific, Torrington, CT) and a total of 1 $\mathrm{mL}$ lactated physiological saline was supplemented during the procedure. The sham procedure involved every step described for the occlusion experiments, except the monofilament was removed immediately after being inserted $5 \mathrm{~mm}$ into the ICA lumen.

For 24-hour survival experiments, animals were re-supplemented with $0.5 \mathrm{~mL}$ of physiological saline 6 hours after waking. For longer survival experiments, animals were supplemented with 
$0.5 \mathrm{~mL}$ of physiological saline within the first 6 , and another $1.0 \mathrm{~mL}$ within the first 30 hours of the MCAO or sham procedure. Weight was monitored daily, and a general neurological score was monitored for welfare purposes. 24,48 or 72 hours after the procedure, animals were placed under deep anesthesia using 5\% inhalant isoflurane, whereby euthanasia was performed by a terminal decapitation procedure.

\subsection{Middle cerebral artery occlusion - rats}

Middle cerebral arterial occlusion was performed at a 60 minute occlusion time as described by Uluç and colleagues ${ }^{237}$ and $\mathrm{Hu}$ and colleagues ${ }^{7}$, and is similar to the procedure for mice, with minor modifications. Briefly, anesthesia was induced by $5 \%$ isoflurane (Piramal Critical Care, Bethlehem, PA) and maintained at $2 \%$ with oxygen for the duration of the procedure. Occlusion was introduced by 2.2-3.0 cm 4-0 monofilament nylon suture bearing a 1.3 or $1.4 \mathrm{~mm}$ silicone tip (Doccol Corp, Redlands, CA) into an arteriotomized external carotid artery and guided 18-20 mm to the middle cerebral artery lumen through the internal carotid artery. The transient occlusion was maintained for 60 minutes. After an initial 7 day recovery period, animal behavior was reassessed for symmetry every week for 4 weeks.

\subsection{Implant design}

All implant designs were generated in SketchUp Make (Trimble Inc., Sunnyvale, CA). Briefly, two columnar platforms designed to fit the curvature of the rat interparietal and parietal bones of the rat skull (Figures 6 and 7), whereby the position of the implant would cover the interparietal suture, approximately $2 \mathrm{~mm}$ rostrally and $2 \mathrm{~mm}$ caudally to the suture using self drilling $1.5 \mathrm{~mm}$ Neuro Screw (Stryker, Kalamazoo, MI) bone screws. A third component, the connector casing plate, was designed to be attachable to the vertical columns using $0.5 \times 3.5 \mathrm{~mm}$ cortical screws (Synthes $\mathrm{GmbH}$, West Chester, PA) The designs were transferred to Ultimaker Cura 15.04 
(Ultimaker, Geldermalsen, NL), and sliced at $0.1 \mathrm{~mm}$ vertical resolution and $0.25 \mathrm{~mm}$ horizontal resolution, and printed in polylactic acid (PLA) at $185^{\circ} \mathrm{C}$.
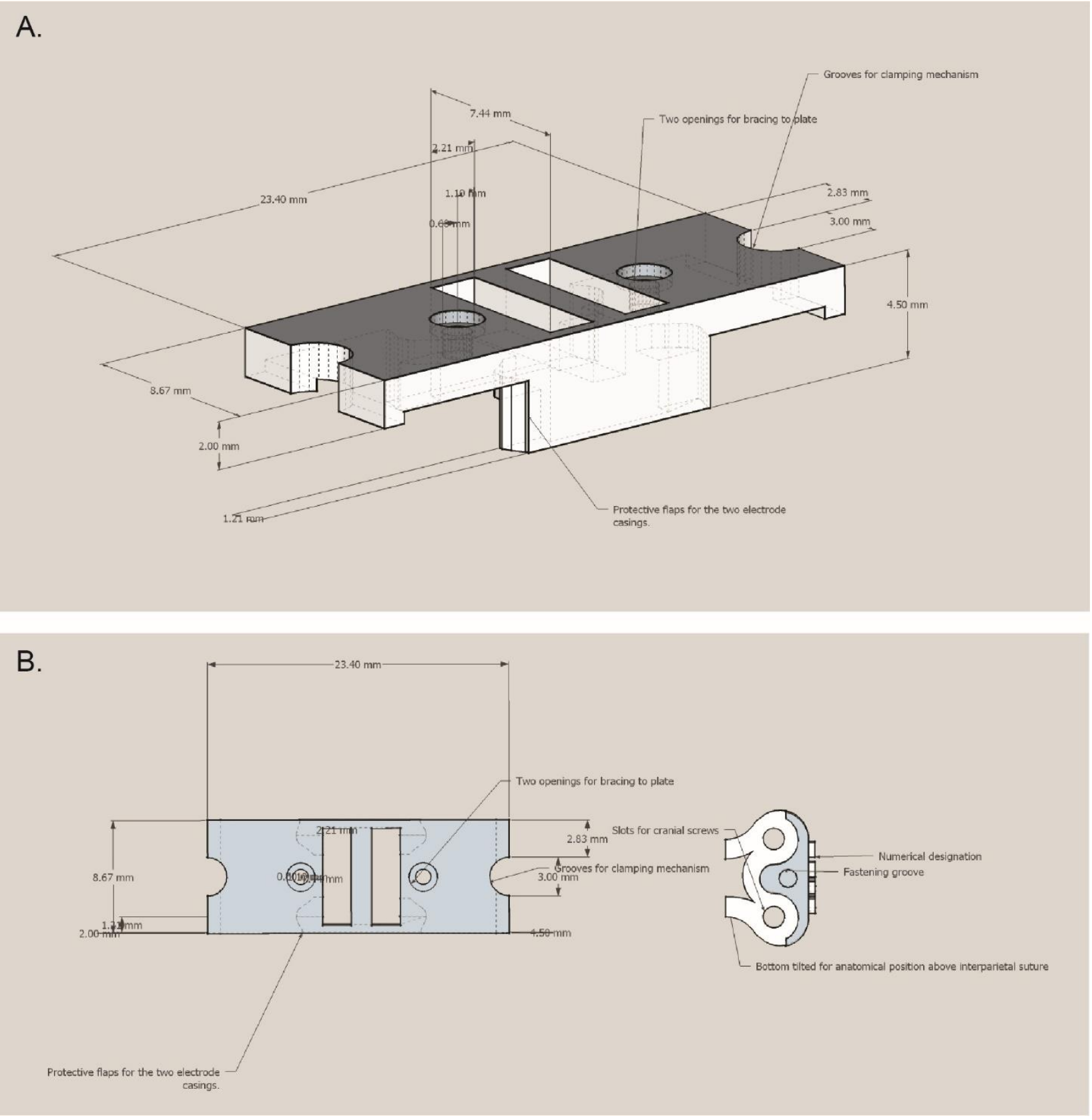

Figure 6. Implant design. A. Three-quarter's profile specifications of the covering plate of the implant. Two grooved slots of $7.44 \mathrm{~mm}$ length and $2.42 \mathrm{~mm}$ width house the electrode casings to fit the specifications of the Grapevine Omnetics Micro+Stim front-end adapter. Lateral to the grooves are two cortical screw slots for bracing to the bracing column. B. Top view of the casing plate (left) and the right bracing column. The bracing columns were designed to fit the width and curvature of the parietal and intraparietal bone. 


\subsection{Chronic electromyograph electrode implantation}

Anesthesia was induced at $5 \%$ and maintained at $1-2 \%$ isoflurane with oxygen and rats were immobilized in a stereotaxic apparatus with gauze coated ear bars, and the sites of incision were injected with lidocaine-HCl (2-4 mg/kg, Aspen Pharmacare, Durban, South Africa) prior to incision. Animals were also administered 5-6 mL of injectable saline (Hospira, Inc. Lake Forest, IL) prior to the procedure to maintain hydration and artificial tear ointment (Rugby Laboratories, Livonia, MI). Similarly to implantation procedures performed in cats previously ${ }^{33}, 8$ pairs of braided Tefloncoated stainless steel wires (AS633, Cooner Wire, Chatsworth, CA) bound to an Omnetics connector (Omnetics Connector Corporation, Minneapolis, MN) were secured to the muscle bellies of 8-16 muscles belonging to the flexor or extensor repertoire of select forelimb or hindlimb pairs by a slip knot. The wires were guided subcutaneously from the implantation site and converged on a custom polylactic acid (PLA, Figure 6) implant coated with a type I collagen digest matrix Collasate (PRN Pharmacol, Pensacola, FL) secured to the temporal bone by $4.5 \mathrm{~mm}$ bone screws (FST, Heidelberg, Germany). Hydration was maintained at the incision sites by $0.5 \mathrm{~mL}$ injectable saline throughout the procedure. Closure was performed with a 3-0 or 4-0 monofilament suture, and aided by GLUture liquid bandage (Abbott Laboratories, North Chicago, IL) or Collasate, and sutures were removed 14 days after the procedure. Recovery was aided for 7 days by subcutaneous administration of Buprenorphine SR (1.2-1.4 mg/kg, Reckitt Benckisser, Berkshire, UK) twice daily, ketoprofen (2-5 mg/kg, Fort Dodge, Lompoc, CA) daily, as well as enrofloxacin (5-10 mg/kg Bayer, Leverkusen, Germany) per orum for 5 days. 

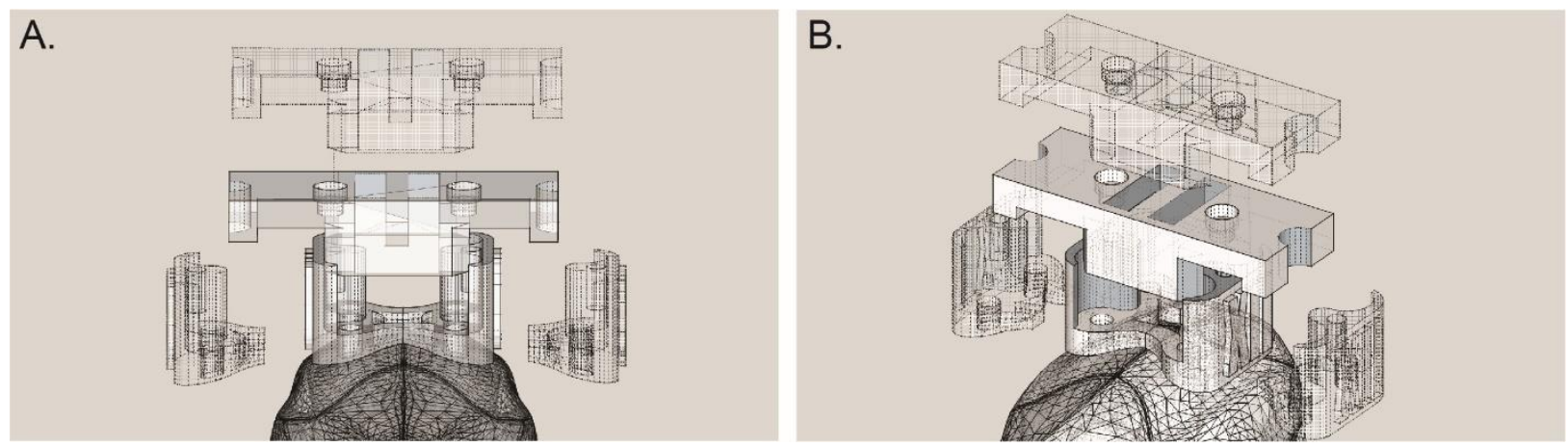

Figure 7. Implant assembly. A. Rear view of the implant positions relative to the bregma. Two bracing columns on each side are implanted first, and the casing plate is implanted by fastening through the cortical screw slots B. Three-quarter's view of the assembly procedure of he implant illustrated in Figure 6.

\subsection{Kinematic analysis}

The duration of each phase of locomotion obtained from the precise foot-placement task was plotted as a function of the corresponding step cycle duration as by Halbertsma and colleagues ${ }^{238}$. Then, the relationship between the locomotor phases and the stepping cycle was extrapolated with the linear regression model $\left(T_{\text {phase }}=B 1+B 2^{\star} T_{c}\right)$ obtained for each limb, where $T c$ represents cycle durations, and ' $T_{\text {phase' }}$ ' is either ' $T_{e}$ ' extensor-related stance or ' $T_{f}^{\prime}$, as the flexor-related swing, and $B 1$ and $B 2$ contain empirical constants (offset and slope) of the regression model. In this model, if $T_{\text {stance }}=B 1+B 2^{*} T_{c}$, then $T_{\text {swing }}=-B 1+(1-B 2)^{*} T_{c}$. The slope (B2) represents the amount of change in phase duration with the change in speed of locomotion. This phase modulation parameter is used to assess the symmetry of the motor system by calculating the asymmetry index (Al), as shown in (1) and (2) for each limb,

$$
A I_{h}=\frac{r-l}{r+l}
$$

and

$$
A I_{v}=\frac{a-p}{a+p}
$$


where ' $a$ ' and ' $p$ ' are the stance phase slopes of anterior and posterior limbs, while ' $P$ and ' $r$ ' represent the stance phase slopes of the left and right limbs. The results from the linear regression model were also utilized as input for the inverse dynamics model described in the model design section. The asymmetry difference between conditions was evaluated by Equation 3 and analyzed using a one-way ANOVA with Tuckey's post-hoc correction.

$$
\Delta A I=\left|A I_{L 9 R 6}-A I_{L 6 R 9}\right|
$$

A second measure, termed the interstance index, was defined as the duration between stance onset of the first and second limb. Similarly to the stance and swing phase characteristic definitions by Halbertsma ${ }^{238}$, and plotted against the cycle durations of the referent limb Four separate values were obtained from each subject corresponding to the interstance slope differences between (1) left and right forelimbs, (2) right forelimbs and hindlimbs, (3) left forelimbs and hindlimbs, and (4) left and right hindlimbs. The interstance difference between conditions was evaluated by Equation 4, where ' $a$ ' and ' $b$ ' represent the first and second locomotor condition of choice. Locomotor differences were evaluated between the left and right lateralized conditions for healthy, as well as paretic locomotion. All differences were analyzed for statistical significance by using the Bootstrap method at a Boot number of 2500 , for a total of 12 subjects. For evaluation of hemiparetic locomotion, a total number of 6 animals was used.

$$
\Delta I S T=\left|I S T_{a}-I S T_{b}\right|
$$

\subsection{Tissue collection and storage}

Brain, as well as tibialis anteriores, extensores digitorum, gastrocnemi, solei and plantares muscles were collected and weighed postmortem, cryopreserved in liquid nitrogen, and stored at $-80^{\circ} \mathrm{C}$ for further analysis. Blood samples were immediately collected in a $\mathrm{K}_{2}$ EDTA coated collection vial (No. 365974 Becton, Dickinson and Company, Franklin Lakes, NJ), and the cellular 
fraction precipitated in a microcentrifuge (Allegra 64R, Beckman Coulter, Brea, CA) at 4500 rcf, for 10 minutes and at $10^{\circ} \mathrm{C}$.

\subsection{Co-immunoprecipitation and Western blotting}

Antibodies for Sirt1, p53, PGC1 a, FoxO3a, p-FoxO3a, and p65 were obtained from Cell Signaling (Danvers, MA). Antibodies for GAPDH, Sirt1 (IP) and Ubiquitin (IP) were obtained from Abcam (Cambridge, MA). All the secondary antibodies for immunoblotting including goat anti-rabbit or goat anti-mouse IgG conjugated with horseradish peroxidase (HRP), were obtained from Jackson ImmunoResearch Laboratories (West Grove, PA). Immunoblotting of the cell lysates were conducted according to established previously ${ }^{239}$. Briefly, the muscle lysates were diluted in $4 \mathrm{X}$ NuPAGE LDS sample buffer (Invitrogen) and denatured at $70^{\circ} \mathrm{C}$ for $10 \mathrm{~min}$. Equal amount of proteins were loaded in a 4-12\% gradient polyacrylamide gel (Invitrogen), and separated by gel electrophoresis. The proteins were transferred to a nitrocellulose membrane (Bio-Rad) and the membranes were blocked in 5\% non-fat milk dissolved in Tris buffered saline with $0.05 \%$ Tween20 (TBS-T) for 1 hour at room temperature and then probed with primary antibodies overnight at $4^{\circ} \mathrm{C}$. This was followed by incubation with secondary antibodies for 1 hour at room temperature. The resulting signals were developed using Amersham enhanced chemiluminescence lighting (ECL) western blotting detection reagent kit (GE Health Care) and exposed to films. The protein signals were captured with a digital camera (Kodak 290). For densitometric analysis, photographs were obtained via KODAK 1D molecular imaging software. 
A.

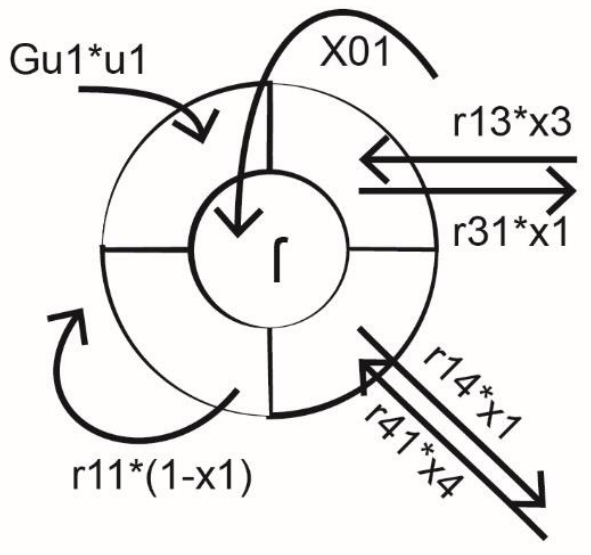

B.

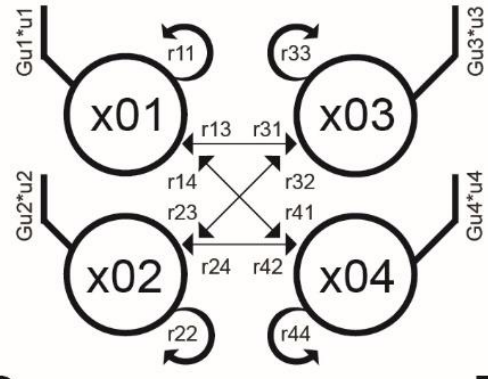

.

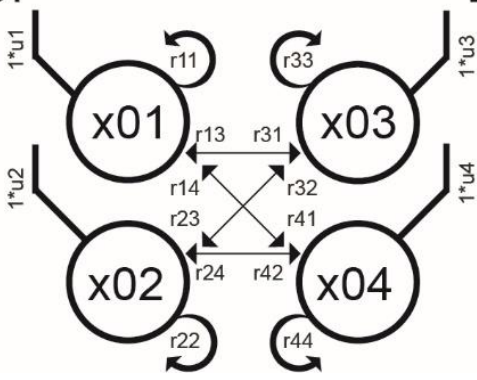

C.

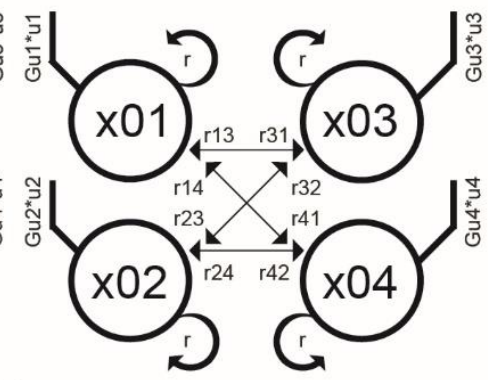

E.

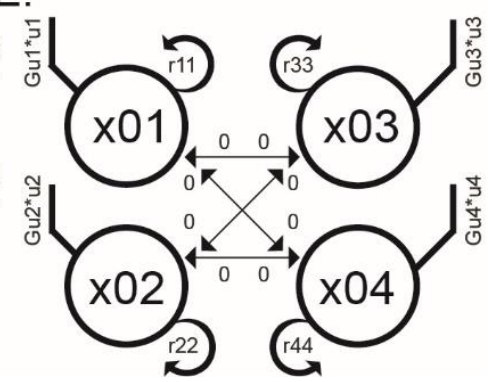

Figure 8. Connectivity scheme of single and multiple integrators. A. Single integrator design. ' $u$ ' represents the tonic drive originating from the midbrain locomotor region and reticular formation. This drive is modulated by the gains ' $G u$ ', and the integration in the integrator is further modulated by the selfsustained firing factors ' $r 11$ ', ' $r 22$ ', ' $r 33$ ', and ' $r 44$ '. ' $r 13$ ' and ' $r 31$ ' are unequal parameters modulating to and fro, respectively, the activity between like integrators (ie. flexor-flexor coupling), while the r41 and r14 scalars are unequal parameters coupling the unlike contralateral integrators (ie. flexor-extensor coupling). The pairing of integrators on each side is termed the model half-center. B. C., D. and E. Architecture differences between the four models. The combined model in B. uses all of the parameter matrices into equation (1). In $\mathbf{C}$. the four self-sustained firing parameters ' $r 11-44$ ' are reduced in a single parameter ' $r$ '. The intrinsic model (C.) relies on the ' $G_{x}{ }^{B L}$ ' and ' $G_{x} U L^{\prime}$ ' matrices alone, and the all extrinsic model (E.) relies on parametric values represented in the ' $G_{u}$ ' and ' $G_{x} U L$ ' matrices alone.

\subsection{Model Description}

The asymmetric locomotor pattern generator was designed as an elaboration on a symmetric central pattern generator model described by Yakovenko in 2010, which, in turn, relies on the classical principle of neural network rhythmogenesis described by the Brown half-center oscillator hypothesis. The pattern generator is separated into two half-centers, each consisting of two integrators representative of the flexor and extensor phase-producing motoneuron populations at the level of the cervical or lumbar enlargements of the spinal column. Phase resetting in this model relies on the integrator's intrinsic ability to integrate inputs until a set threshold, after which activity is shifted to the next integrator within the half-centers. Here, the activity between integrators in 
the half-centers is mutually exclusive, modeling the behavior whereby the extensor driven stance excludes flexor driven swing. This architecture allows the half-center oscillations to be reliant on a non-scalar all-or-nothing rule. The regulation of oscillation between the left and right halfcenters, however, is reliant on several scalar factors which influence the rate of integration in a given oscillator by the activity of a contralateral integrator, termed state-dependent activity. The interconnectivity parameters are divided into separate parametric subsets (Table 2), which are used for statistical analyses. As such, the model can be expressed as a system of differential equations of extrinsic and intrinsic interactions of the system.

$$
x^{\prime}=x_{0}+G_{u} u+G_{x}^{U L} x+G_{x}^{B L}(1-x) \mid x>0
$$

The extrinsic parameters of the model are set of scalar factors modulating a descending drive such as the one from the midbrain locomotor region $(u)$, and are representative of higher centers of the motor hierarchy (ie. the motor cortex, $x_{0}$ ), which are modified by a second scalar factor representing the modulatory gain from intermediate centers (such as the basal ganglia and red nuclei, termed $G_{u}$ ). A parameter that models self-sustained firing imposed by the ipsilateral connectivity between spinal motor neuron populations, which in mammals is represented by the Renshaw cells, V1, Chx10+, and Gata2/3 interneurons ${ }^{36}$, and is represented by the ' $G_{x}$ UL' matrix in the following, as well as previous iterations of the model ${ }^{234}$. It is important to note that the model presented herein does not represent a cellular model of rhythmogenesis, but rather takes the behavior of all of these interneuronal populations as a scalar, meaning that the intrinsic parameters between the half-centers may well speed-up or slow-down integration within, say, the flexor group, regardless of the excitatory or inhibitory phenotype of the individual neurons.

$$
G_{x}^{B L}=\left[\begin{array}{llll}
0 & 0 & r 13 & r 14 \\
0 & 0 & r 23 & r 24 \\
r 31 & r 32 & 0 & 0 \\
r 41 & r 42 & 0 & 0
\end{array}\right]
$$


Previous iterations of the model employed few parameters representing the commissural connectivity between the half-centers, but these scalar values were kept the same for left-right and right-left connectivity. Biologically, however, these inter-center connections may be modulated predominately by the contralateral projections from the motor cortex to spinal commissural interneurons, and it is not necessarily true that this scalar value will be kept the same if the higher centers of the motor hierarchy are unilaterally injured. For the purpose of this combined model, the current asymmetric model assumes that the intrinsic connectivity between the half-centers is unequal. The resulting connectivity allows for the phasic regulation to be asymmetric as well, meaning that during numerical evaluation of the differential equation

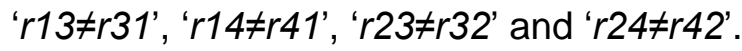

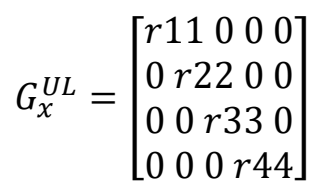

\begin{tabular}{|c|c|}
\hline Parameters & Type \\
\hline U01, U02, U03, U04 & Input signals \\
\hline$x 01, x 02, x 03, x 04$ & Constant bias, extrinsic \\
\hline Gu1, Gu2, Gu3, Gu4 & $\begin{array}{c}\text { Gain of input signal, } \\
\text { extrinsic }\end{array}$ \\
\hline$r 11, r 22, r 33, r 44$ & Self-sustainment \\
\hline $\begin{array}{c}r 13, r 14, r 23, r 24, r 31, \\
r 32, r 41, r 42\end{array}$ & $\begin{array}{c}\text { Bilateral coupling, } \\
\text { intrinsic }\end{array}$ \\
\hline $\begin{array}{c}x 01, x 03, r 11, r 33, r 13, \\
r 14, r 31, r 32\end{array}$ & Flexor-Related \\
\hline $\begin{array}{c}x 02, x 04, r 22, r 44, r 23, \\
r 24, r 41, r 42\end{array}$ & Extensor-Related \\
\hline
\end{tabular}

Table 2. Parameter nomenclature. For the purposes of statistical analysis of various schemes of driving the proposed central pattern generator architecture, parameters were grouped into separate types related to their nature. The input signals ' $U$ ' were kept as constants, while parameters designated ' $x O$ ', ' $G u$ ' and ' $r$ ' were the unknown variables.

\subsubsection{Extrinsic versus intrinsic models}

In order to compare the stability of the modeled systems, four separate connectivity architectures (Table 3) were designed for the evaluation of locomotor behavior composed of four extrinsically and intrinsically connected integrators, as an elaboration of a single integrator model with state- 
dependent activity (Figure 8A). The first, combined, model employs both intrinsic and extrinsic connectivity of the locomotor hierarchy in order to produce the proper locomotor pattern (Figure 8B). A second, similar, model was made comparable to the first, with the exception that the selfsustained parameters were kept the same for all integrators (Figure $\mathbf{8 C}$ ). The third model relies on the later experiments shown by Thomas Graham Brown whereby the cerebrospinal inputs are set to 1 , while the entire process of pattern generation relies on the intrinsic connectivity of the spinal cord (or rather, on the ' $G_{x}{ }^{B L}$ ' matrix alone, Figure $8 D$ ). The fourth model relies on the paradigm proposed by Sir Charles Scott Sherrington, whereby the alternation of flexion and extension is modeled as a mutually exclusive process that does not rely on the intrinsic connectivity of the spinal cord, but is rather driven by descending input originating from higher centers of the motor hierarchy. This model is composed of the extrinsic parameters alone, and assumes the intrinsic connectivity of the oscillators to be zero (disregarding connectivity of the spinal interneuronal populations, Figure 8E).

\begin{tabular}{|c|c|c|}
\hline Model Type & Parameter Characteristics & No. of Parameters \\
\hline Expanded & Extrinsic, self-sustained and intrinsic & 20 \\
\hline Reduced & Extrinsic and intrinsic & 17 \\
\hline Brown Type & Intrinsic and Self-Sustained & 12 \\
\hline Sherrington Type & Extrinsic and Self-Sustained & 12 \\
\hline
\end{tabular}

Table 3. Model types. Each model type represented in Figure 8 is described according to parametric characteristics and the total parameter number.

\subsubsection{Optimization}

A custom script using MATLAB (MathWorks, Natick, MA) was generated for the performance of the optimization. An optimization function that utilizes the fminsearch MATLAB function, and employing a Nelder-Mead linear minimization algorithm was used to find optimal solutions to the asymmetric model with a state tolerance of $5.0 \times 10^{-10}$, and a function tolerance of $5.0 \times 10^{-10}$. The optimization process minimizes the error, expressed as a cost function, and is composed by three 
error parameters. The first cost function compares similarity between the simulated and experimental slope of the phase characteristic data, with greater similarity accounting for lower error. The second (alternative) cost function produces a high cost value in the event that the model produces an unequal number of stance events between the simulated limbs, as this is the defining parameter of locomotor behavior, and one which has been difficult to simulate in previous iterations of the model ${ }^{240}$. The individual error functions are further selected through alternation, whereby suboptimal behavior in either function potentiates cost optimization through the most incongruent error functions, while reducing the cost weight through the other function. Simulations were obtained from initial inputs generating absolutely symmetrical locomotion, and based on parameters obtained in the asymmetric optimization paradigm for symmetric unconstrained walking behavior and for short range of locomotor speeds. Then, the suboptimal solution was used as initial input for further optimization using longer simulation times, generating a better fit for a wider range of locomotor speeds. In some cases, the stable state space was not obtainable using wider cycle time ranges. In such cases, the weights of the individual error functions were altered, and the differential equation was passed again through the optimization process to obtain a more optimal solution (Figure 9). The initial conditions represented a parameter set which produces a perfectly symmetrical oscillatory output, with a duration bias on the integrators replicating extensor function (Equation 4). For the purpose of these experiments, the initial conditions were taken to be the same for all simulations of subject data within the experimental model groups. For the purpose of optimizing the expanded (combined Sherrington-Brown type) 20 parameter model, two different sets of initial conditions were taken. An absolutely symmetrical initial condition set $\left(\mathrm{IC}^{\mathrm{S}}\right.$, Equation 8$)$ is a solution set of the central pattern generator ordinary differential equation (CPG-ODE) which produces equivalent stance and swing phase characteristics - that is to say that the stance and swing duration is equal at different cycle speeds, whereby they represent exactly half of the cycle duration. 
A.

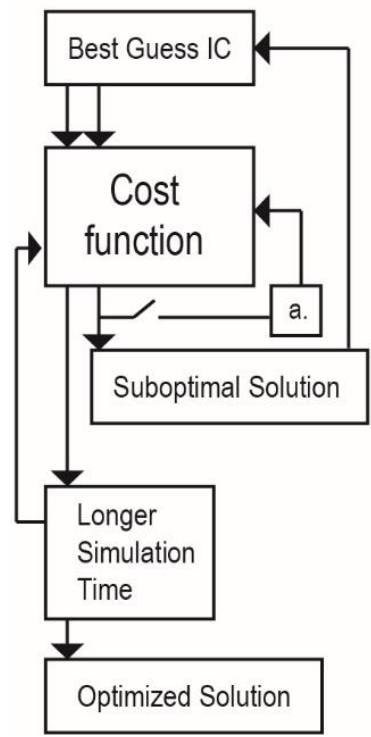

B.

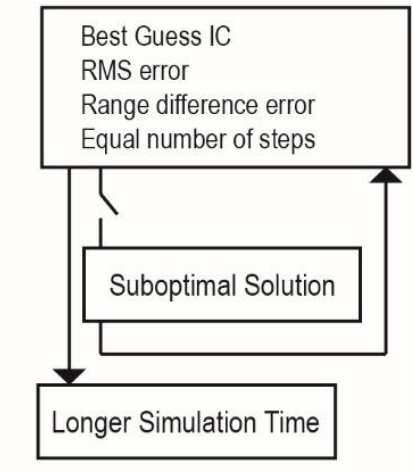

Best Guess IC RMS error

Range difference error Equal number of steps
Figure 9. Optimization. A. Optimization scheme for obtaining parametric weight for all model types. The initial best guess values were selected for each different model type. The cost function $\mathbf{B}$. is then used to obtain more optimal solutions, which are further passed as initial conditions for simulations of a wider locomotor speed range (longer simulation time) to obtain more optimal solutions. An alternative cost function (a.) can be used to correct poor task-space solutions before continuing to longer simulation times. B. Scheme illustrating the cost function. Once a bad solution is obtained from the Nelder-Mead Simplex optimization, the suboptimal (bad) solution is passed through a cost bias step to avoid suboptimal output task-space, and then passed again through longer simulation times to obtain a more optimal solution.

$$
x^{\prime}=\left[\begin{array}{llll}
1 & 1 & 1 & 1 \\
1 & 1 & 1 & 1 \\
1 & 1 & 1 & 1 \\
1 & 1 & 1 & 1
\end{array}\right]+\left[\begin{array}{llll}
1 & 1 & 1 & 1 \\
1 & 1 & 1 & 1 \\
1 & 1 & 1 & 1 \\
1 & 1 & 1 & 1
\end{array}\right] u+\left[\begin{array}{llll}
1 & 0 & 0 & 0 \\
0 & 1 & 0 & 0 \\
0 & 0 & 1 & 0 \\
0 & 0 & 0 & 1
\end{array}\right] x+\left[\begin{array}{llll}
0 & 0 & 0 & 0 \\
0 & 0 & 0 & 0 \\
0 & 0 & 0 & 0 \\
0 & 0 & 0 & 0
\end{array}\right](1-x) \mid x>0
$$

A second set of initial conditions (Equation 9) was a solution which produces a flexor-bias (ICF), whereby the inputs to the integrators simulating activity of the flexor phase are significantly greater than the inputs to the integrators simulating the extensor phase. This solution to the CPG-ODE generates a longer stance duration, akin to the behavior observed empirically, whereby the duration of the stance phase is significantly longer in duration than the one of the swing phase, and is equal on both limbs.

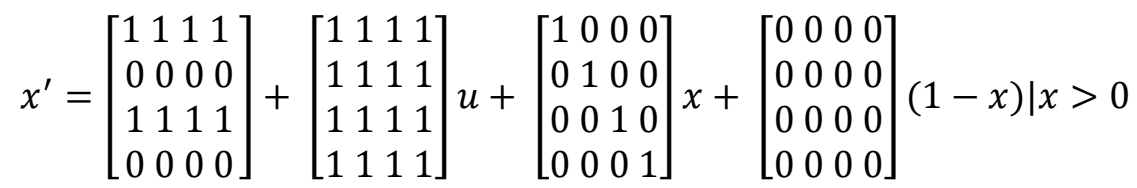

The solution sets obtained from optimizations from Equation 8 and Equation 9 were compared for weight distribution among parametric subsets. This evaluation was performed for healthy as well as hemiplegic locomotor outputs. Lastly, hemiplegic solution sets of the CPG-ODE were taken as 
initial conditions in order to evaluate whether the fminsearch function was capable of capturing a mode of least resistance in which the hemiplegic solution could be readapted in order to produce a healthy output. These hemiplegic-to-healthy solution sets hypothetically represent a parametric solution closest to producing healthy locomotion in task-space, and the novel solution may represent the manner of retraining an impaired CPG architecture in order to produce the desired healthy output. Lastly, initial conditions of either $30 \%, 60 \%$ or $220 \%$ intrinsic parameter contribution were chosen as best guesses after a systematic analysis of different extrinsic-tointrinsic parameter contributions, which were obtained by different ' $G \times B L$ ' matrices as:

$$
G x_{B L}^{30 \%}=\left[\begin{array}{cccc}
0 & 0 & 0.3 & 0.3 \\
0 & 0 & 0.3 & 0.3 \\
0.3 & 0.3 & 0 & 0 \\
0.3 & 0.3 & 0 & 0
\end{array}\right] ; G x_{B L}^{60 \%}=\left[\begin{array}{cccc}
0 & 0 & 0.6 & 0.6 \\
0 & 0 & 0.6 & 0.6 \\
0.6 & 0.6 & 0 & 0 \\
0.6 & 0.6 & 0 & 0
\end{array}\right] ; G x_{B L}^{220 \%}=\left[\begin{array}{llll}
0 & 0 & 2.2 & 2.2 \\
0 & 0 & 2.2 & 2.2 \\
2.2 & 2.2 & 0 & 0 \\
2.2 & 2.2 & 0 & 0
\end{array}\right]
$$

The different parametric distribution for initial conditions is summarized in Figure 10, where the topmost row represents the axes of contralateral coupling among extrinsic parameters (left), selfsustainment parameters (middle) and intrinsic parameters (right).

First, different model architectures were subjected to the optimization scheme in Figure 9 in order to evaluate the feasibility of error reduction in an expanded asymmetric model where contralateral left-to-right and right-to-left half-center couplings are unequal. Then, solutions of the ODE reproducing either healthy (Figure 11A, Optimization I) or hemiparetic (Figure 11A, Optimization II) rhythmic outputs from each corresponding initial condition set were passed through parametric analysis (see Section 4.10.5. for statistical analysis) and in order to generate Figure 23. Further, the expanded 20 parameter (combined Sherrington-Brown model) was used to produce similar solutions reproducing healthy (Figure11B Optimization I) or hemiparetic (Figure 11B Optimization II) gait patterns. Once the hemiparetic solutions were obtained, they were used as initial conditions for a third optimization process (Figure 11B, Optimization III), and in order to obtain a readapted solution (see Section 4.10.2.1.), or rather, the solution set which lies closest 
to a secondary set in task-space which reproduces healthy output. The method for analyzing the different parametric distributions and the differences between solutions is illustrated in Figure 11.

Extrinsic Self-Sustainment Intrinsic
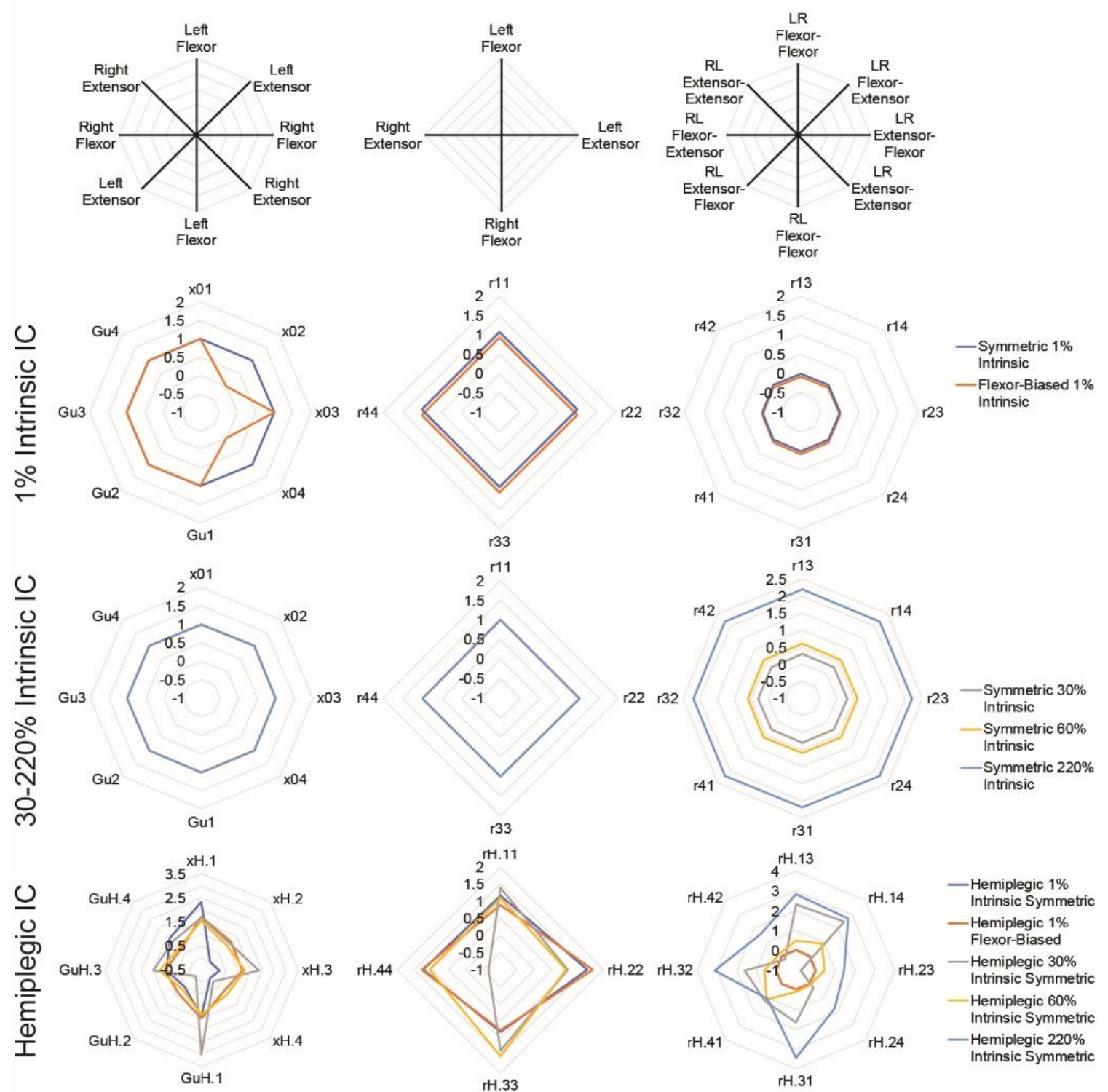

Figure 10. Initial conditions. Radar plots of parametric sets used as initial conditions for subsequent optimization. Top row, right - relationship axes of input gain signals ' $G u$ ' and constant bias ' $x 0$ '; top row, middle - left-right flexor-flexor and extensor-extensor relationships of self-sustainment parameters; top row, left - right-left and left-right extensor and flexor relationships for intrinsic parameters. Second row, parametric weight of the two different $1 \%$ intrinsic contribution initial conditions (IC). Third row, parametric weight distributions for the initial conditions of different intrinsic parameter contributions. Dark gray trace symmetric $30 \%$; yellow trace - symmetric $60 \%$; turquoise trace - symmetric $220 \%$ intrinsic ICs. Bottom row, hemiparetic output solutions used as initial conditions for readaptation analyses. Navy trace $-1 \%$ intrinsic symmetric; orange trace $-1 \%$ intrinsic flexor-biased; gray trace $-30 \%$ intrinsic; yellow trace $-60 \%$ intrinsic; turquoise trace $-220 \%$ intrinsic. 
A.

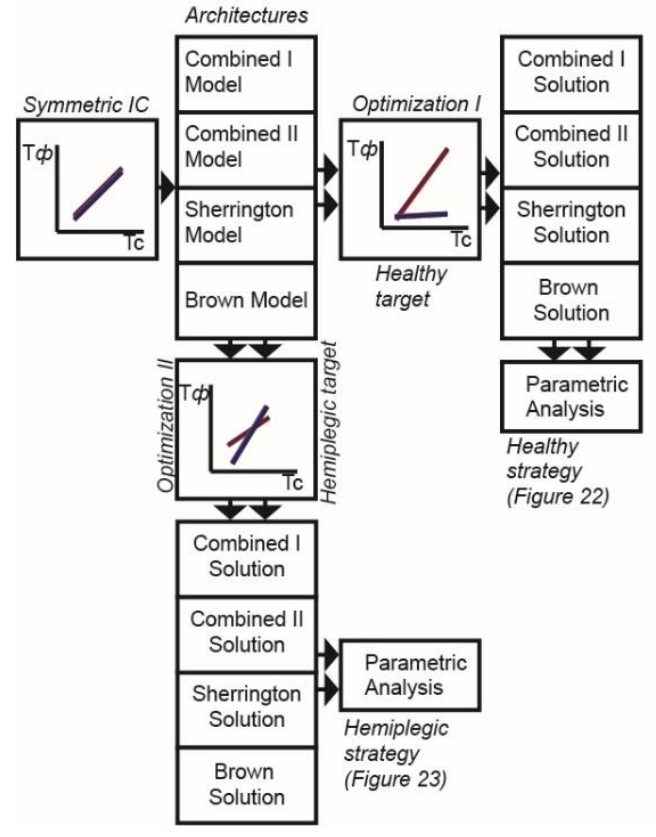

B.

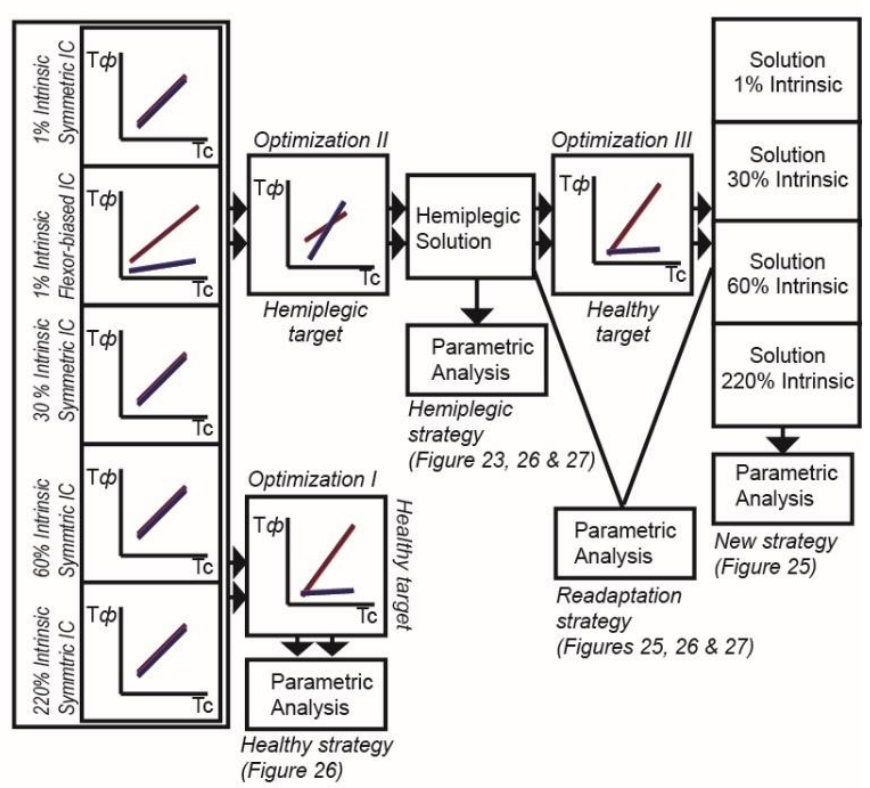

Figure 11. Analysis scheme. A. Method for generating and analyzing solutions from different model architectures from equal or equivalent initial conditions. Absolutely symmetric initial conditions producing stance and swing phases of equal duration were taken as initial conditions for all optimizations. Then, numerical ordinary differential equation (ODE) solutions were obtained by Nelder-Mead Simplex optimizations for healthy or hemiplegic targets. Parametric weight differences between the healthy left and right lateralized gait were used for analysis of the different modes of driving the CPG in healthy locomotor behaviors. In order to evaluate the ODE solutions simulating hemiplegic gait, the different model architectures were used to generate locomotor outputs of hemiplegic gait. B. Method for generating and analyzing numerical solutions obtained from different initial conditions. Symmetric initial conditions (ICS), and flexor-biased initial conditions (ICF) were passed through an optimization process to target hemiplegic gait behavior. The solutions of hemiplegic gait were then taken as initial conditions, and passed through a second optimization process targeting healthy gait.

\subsubsection{Optimization from solutions of hemiplegic output (readapted solutions)}

In order to evaluate whether subsequent hemiplegic solutions obtained from either Equation 8 or Equation 9, ODE solution sets which reproduce hemiparetic rhythmic patterns were used as initial conditions, where the individual 'hemiparetic' parameters (designated ' $\mathrm{xH}$ ' through ' $r \mathrm{H}$ ', Equation 10) were substituted in corresponding positions of the ordinary differential equation where:

$$
x 0=\left[\begin{array}{l}
x H .1 \\
x H .2 \\
x H .3 \\
x H .4
\end{array}\right] ; G u=\left[\begin{array}{l}
G u H .1 \\
G u H .2 \\
G u H .3 \\
G u H .4
\end{array}\right] ; G x=\left[\begin{array}{cccc}
r H .11 & 0 & 0 & 0 \\
0 & r H .22 & 0 & 0 \\
0 & 0 & r H .33 & 0 \\
0 & 0 & 0 & r H .44
\end{array}\right] ; G x B L=\left[\begin{array}{ccccc}
0 & 0 & r H 13 & r H .14 \\
0 & 0 & r H 23 & r H .24 \\
r H .31 & r H .32 & 0 & 0 \\
r H .41 & r H .42 & 0 & 0
\end{array}\right] .
$$


These values were taken from hemiplegic solutions of $1 \%$ intrinsic parameter contribution, or from solutions with increasing intrinsic parameter contribution (Figure 10, bottom row). The cost minimization target for these investigations was taken as the healthy locomotor output.

\subsubsection{Output target}

The output target for the optimization was selected as the empirically obtained stance and swing phase characteristics from healthy locomotion, as well as paretic locomotion from rats which had undergone a unilateral transient middle cerebral artery occlusion. An optimized solution to the differential system was one which had produced minimal root-mean-square error between the simulated and empirically obtained regression models, was within the mean range of locomotion, and produced an equal number of integrations on all integrators within a given cycle time. Finally, an objective error parameter was evaluated across the full range of cycle times to assess the effectiveness of each optimization iteration. As the locomotor condition which challenges the paretic side (left lateralization - L9R6) was found to produce significant phase modulation alterations of the stance and swing phases, this condition was selected for the purpose of evaluating model solutions which produce paretic gait.

\begin{tabular}{|c|c|}
\hline Parametric Subset & Parameters \\
\hline extrinsic flexor & x01, x03, Gu1, Gu3 \\
\hline extrinsic extensor & x02, x04, Gu2, Gu4 \\
\hline self-sustained flexor & $\mathrm{r} 11, \mathrm{r} 33$ \\
\hline self-sustained extensor & $\mathrm{r} 22, \mathrm{r} 44$ \\
\hline intrinsic flexor-flexor & $\mathrm{r} 13, \mathrm{r} 31$ \\
\hline intrinsic flexor-extensor & $\mathrm{r} 14, \mathrm{r} 32$ \\
\hline intrinsic extensor-flexor & $\mathrm{r} 23, \mathrm{r} 41$ \\
\hline intrinsic extensor-extensor & $\mathrm{r} 24, \mathrm{r} 42$ \\
\hline
\end{tabular}

Table 4. Alternate parametric subgroups. Subsets of flexor and extensor related parameters were subgrouped for their extrinsic, self-sustained, or intrinsic characteristics, and used for stability analysis. 


\subsubsection{Stability}

In order to evaluate the stability of the proposed asymmetric central pattern generator model, noise of different signal-to-noise ratios (SNR) was introduced to either the extrinsic, self sustainment or extrinsic parametric subsets, at either 30,20 , or 10 SNR, where the greater numbers imply greater power of signal to noise for a total number of 15 trials (Figure 27). Furthermore, stability analysis was performed along alternative subset axes, where flexor and extensor related parameters were grouped along their parametric subset characteristics summarized in Table 4. Noise was introduced through the MATLAB built-in function awgn, as follows:

$$
S N R=\frac{\sigma_{\text {signal }}^{2}}{\sigma_{\text {noise }}^{2}}
$$

The dataset of perturbed parameters was then used as input in the ordinary differential equation model, and the output of the set was evaluated by the error function used for optimization (Figure 18D) described in Section 4.10.2. Optimization. The resulting errors from the perturbed parametric sets were plotted on a logarithmic scale in order to evaluate the stability of each solution in response to noise (Figure 28). The flexor and extensor subsets represented in Table 3 were subjected to 10 SNR noise levels, and analyzed for stability by using the error function represented in Figure 18D and summarized in Section 4.10.2. The resulting error from the perturbed solution sets was subtracted from the error of the optimized solution, and the results were plotted on a logarithmic scale (Figure 29).

Solution sets from a single subject for $1 \%$ or $30 \%$ intrinsic parameter contribution, relative to extrinsic parameter contribution, were selected for further analysis, and the solution space within $10 \%$ of a pair of extrinsic, self-sustained, and intrinsic parameters contributing to a single halfcenter was analyzed for root-mean-square deviation from the optimized solution. The results were plotted in a heat-map, whereby percentile RMS deviation from optimum was plotted against 
parametric perturbations of $10 \%$ or $20 \%$, and in order to sample the solution space within the vicinity of optimized solutions.

\subsubsection{Statistical analysis}

Asymmetry indices evaluated from conditions L9R6 and L6R9 $(\triangle \mathrm{Al})$ were tested with a one-way ANOVA $(\alpha=0.05)$ and post-hoc t-tests with conservative Bonferroni correction (adjusted $\alpha=$ 0.0125) using the 'anova' and 'multcompare' functions in Matlab. Statistical analysis for parametric deviation among different model solutions was performed using effective size variation, in which extrinsic, self-sustained and intrinsic parameter subsets were taken as separate groups. For the extrinsic Sherrington-type model, the ' $G u$ ' and ' $x 0^{\prime}$ matrices were taken as the extrinsic parameter set, and their variation was compared between the ' $G_{x}{ }^{U L}$ ' matrix, which was taken as the self-sustained parameter set. For the intrinsic Brown-type model, the intrinsic connectivity parameters ' $G_{x}{ }^{B L}$ ' were assessed against the intrinsic parameters for the other two models. The effective size value was obtained by comparing the size variation between the two asymmetric conditions (Equation 12), and significance was assessed using the bootstrap method at a sampling rate of 100,000 boots.

Statistical analysis was also performed on individual parameters for the fully expanded model $(n=20)$, as well as the conservative all intrinsic or all extrinsic models $(n=12)$, and the effective size difference $(\delta E S)$ was assayed by using Equation 12. All statistical analyses were performed between healthy locomotor output of right- or left-favored gait, as well as between healthy and hemiparetic locomotor output of the locomotor condition challenging the left (hemiparetic) side of longer left interstride length (L9R6).

$$
\delta E S=2 \frac{\sum_{j}^{n}\left|x_{j}^{\text {condition } 1}-x_{j}^{\text {condition } 2}\right|}{N_{\text {sub }}\left(\sigma_{x j}^{\text {condition } 1}+\sigma_{x j}^{\text {condition } 2}\right)}-2 \frac{\sum_{j}^{n}\left|n_{j}^{\text {condition } 1}-n_{j}^{\text {condition } 2}\right|}{N_{\text {sub }}\left(\sigma_{n j}^{\text {condition } 1}+\sigma_{n j}^{\text {condition } 2}\right)}
$$




\section{RESULTS}

\subsection{Transient ischemic stroke induces dorsiflexor-dependent gait lateralization}

All asymmetry indices were calculated using Equation 1 and 2 from all subjects individually. Generally, forelimb stance slope was diminished for the side to which the locomotion condition was favored, consistent with the notion that the stance phase on the preferred side tended to occupy a greater portion of the step cycle. The difference between groups was significant $(p=$ 0.002). The anterior horizontal asymmetry index $(\Delta a A / h)$ corresponding to the asymmetry between forelimbs was significantly different $(p=0.006)$ between the left-favored (L6R9) and the right-favored (L9R6) conditions (Figure 12B and 14A). The difference between the conditions for the right vertical asymmetry index $(\Delta r A l v)$ showed a trend, but it was not significantly different from zero $(p=0.031, \alpha=0.0125)$.

Locomotor asymmetry was also evident in EMG recordings from in vivo locomotion of healthy test subjects (Figure 13B). The muscular activation of consecutive proximal-to-distal muscles, characteristic of the swing onset was identified beginning in the cleidobrachialis, and continuing into peak activations of the extensor carpi radialis and extensor digitorum carpi. The peak activation of the latissimus dorsi and palmaris longus was associated with the onset of the stance phase (Figure 13B). The peak activity of these muscle groups is observably different between the left lateralized (L9R6) and right lateralized (L6R9) locomotor behaviors, and from the locomotor condition of symmetric left and right stride lengths (S15). 
A.

Phase Characteristics - L9R6

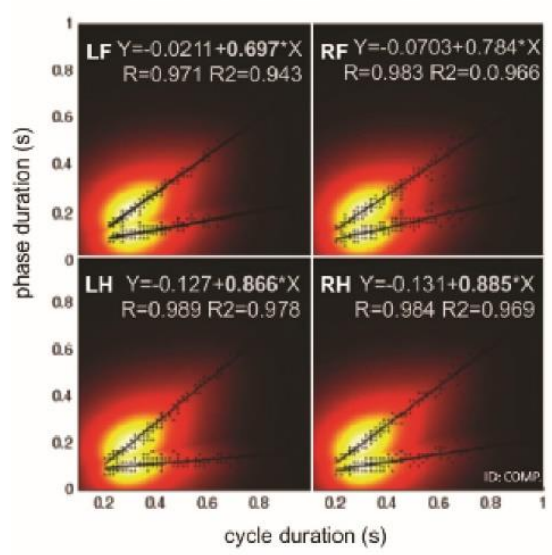

B.

(4) $\triangle A I=\left|A I_{L Q R 6}-A I_{L G R 9}\right|$

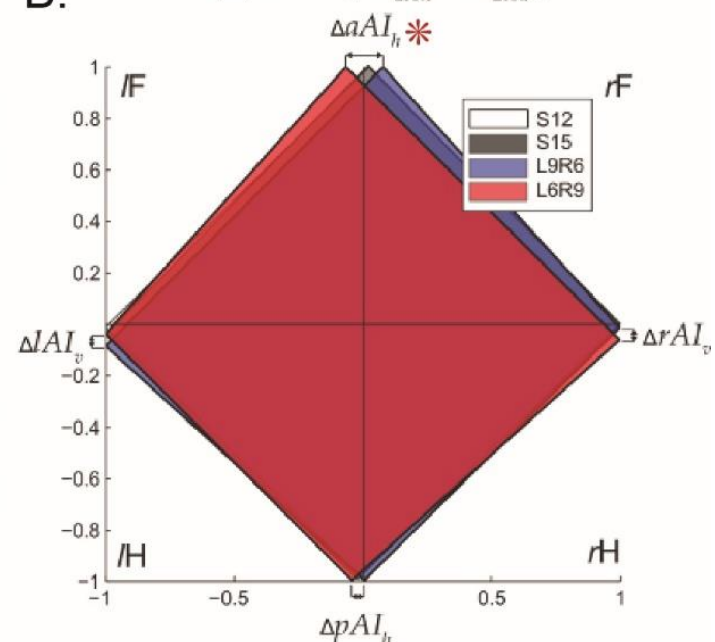

Figure 12. The asymmetric walkway distinguishes lateralized gait. A. Heatmap of locomotor phase (stance and swing) characteristics during healthy left-preferred locomotion. The peg arrangement was such that a longer stride length was imposed on the right side (L6R9, legend). B. Asymmetry indices obtained using Equations 1 and 2 were plotted to obtain a visual representation of horizontal and vertical asymmetry. $\triangle I A I V$ - left vertical asymmetry index change; $\Delta r A I V$ - right vertical asymmetry index change; $\Delta a A l h$ anterior horizontal asymmetry index change; $\triangle p A l h$ - posterior horizontal asymmetry index change.

Force-sensor data from rat locomotion on the precise foot placement task revealed that the onset of paretic limb placement is delayed in subjects which underwent the transient middle cerebral artery occlusion procedure. Chronic electromyographic recordings from rat locomotion revealed a delay in the period of activation of the dorsiflexor group composed of extensor digitorum, extensor carpi radialis, triceps lateralis and triceps longus, during lateralized locomotion which challenges the paretic side, but was not evident in the lateralized locomotor condition which challenges the non-paretic side (Figure 15 and 16). Furthermore, the offset delay on the paretic limb accounts for approximately $10 \%$ of the cycle period, and is associated with the delayed activation of the muscle groups in hemiparetic locomotion. 
A.
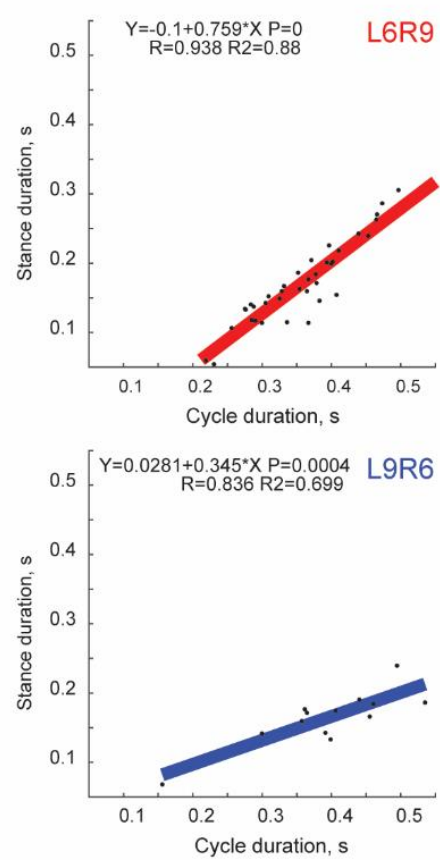

B.

B. Swing Onset
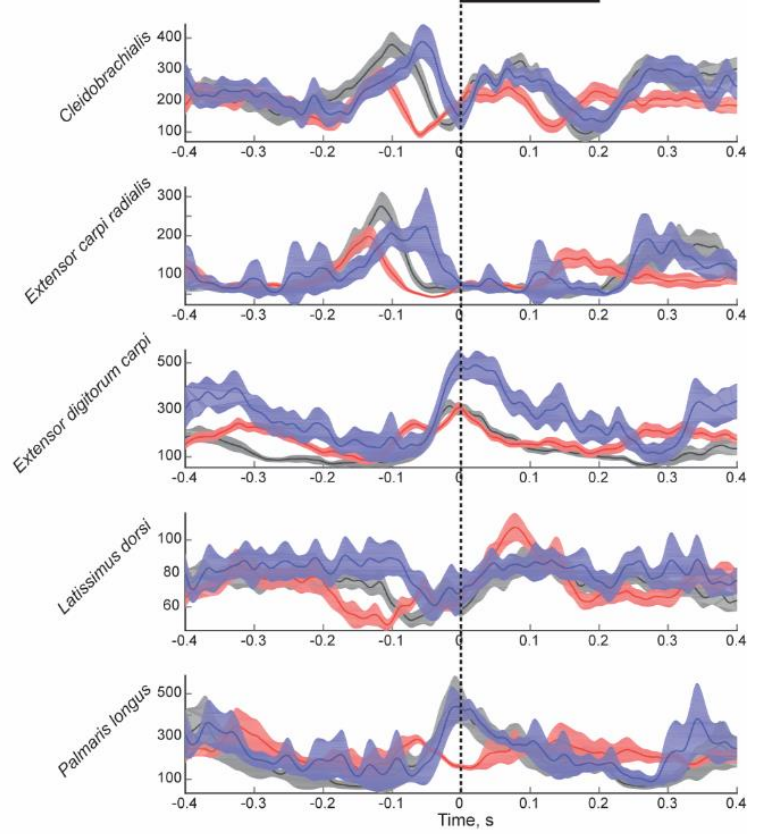

Figure 13. In vivo electromyographic (EMG) recordings of healthy symmetric and asymmetric locomotion. A. Stance phase modulation during right-favored (top) or left-favored (bottom) locomotion. During left-favored gait, the slope of the regression line produced at a given range of locomotor speeds is less steep than the slope of the stance phase regression during right-favored gait. B. EMG recordings from different forelimb muscles demonstrates the classical proximal-to-distal sequential activation of muscle groups. L6R9 - right-favored locomotor condition with a $r S L$ of $9 \mathrm{~cm}$ and ISL of $6 \mathrm{~cm}$; L9R6 - left-favored locomotor condition with a rISL of $6 \mathrm{~cm}$ and ISL of $9 \mathrm{~cm}$; $S 15$ - symmetric locomotor condition of a $I S L$ of $7.5 \mathrm{~cm}$ and $I S L$ of $7.5 \mathrm{~cm}$. The blue traces - left-favored locomotor condition; red traces - right-favored locomotor condition.
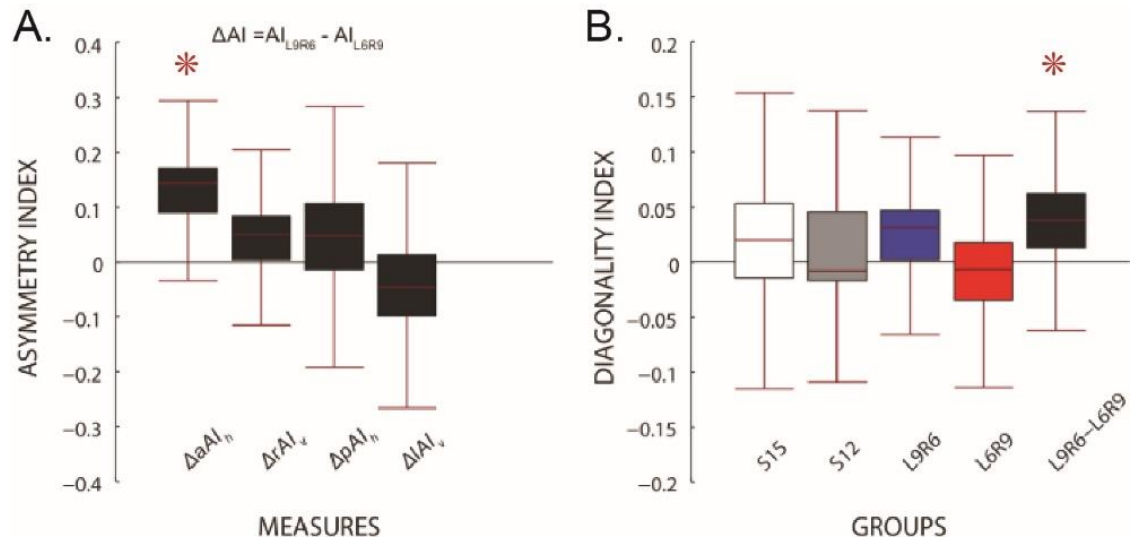

Figure 14. Analysis of asymmetric measures. A. Absolute differences between left-favored and rightfavored gait indices tested with a one-way ANOVA with post-hoc t-test correction by the Bonferroni method. The change in forelimb asymmetry between left-favored and right-favored gait is significantly different. $\mathbf{B}$. Diagonality index distribution between symmetric and asymmetric gait tested using a one-way ANOVA with Bonferroni correction. While neither condition showed a trend for significance, the difference between the diagonality index of left and right-favored gait was found to be significantly different (black plot). 
A.

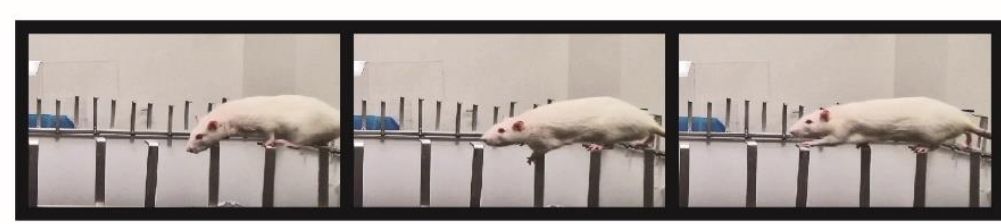

B.

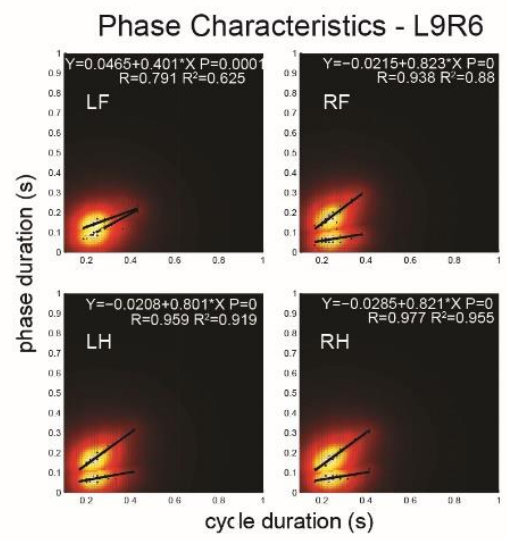

C.

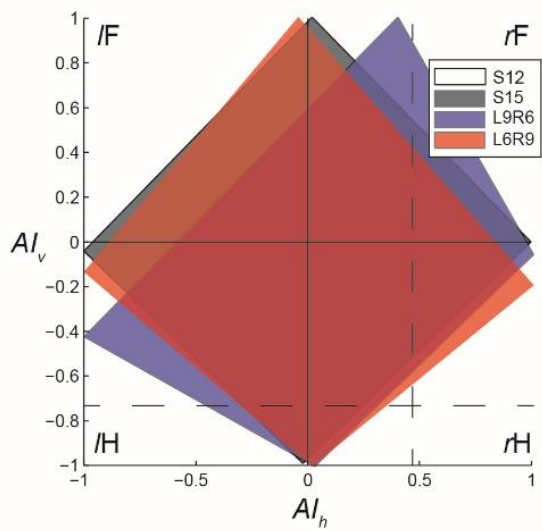

Figure 15. Locomotor phase characteristics after transient unilateral ischemic brain injury. A. The asymmetric walkway induces an abnormal swing behavior on the condition which challenges the paretic corporeal side. B. Phase characteristics of stroked locomotion are altered on the paretic side, whereby the duration of the swing phase exceeds the duration of the stance phase at slower locomotor speeds. C. Asymmetry indices obtained using Equation 1. The asymmetry induced by the L9R6 locomotor condition is significantly different from the locomotor behavior of healthy rats.
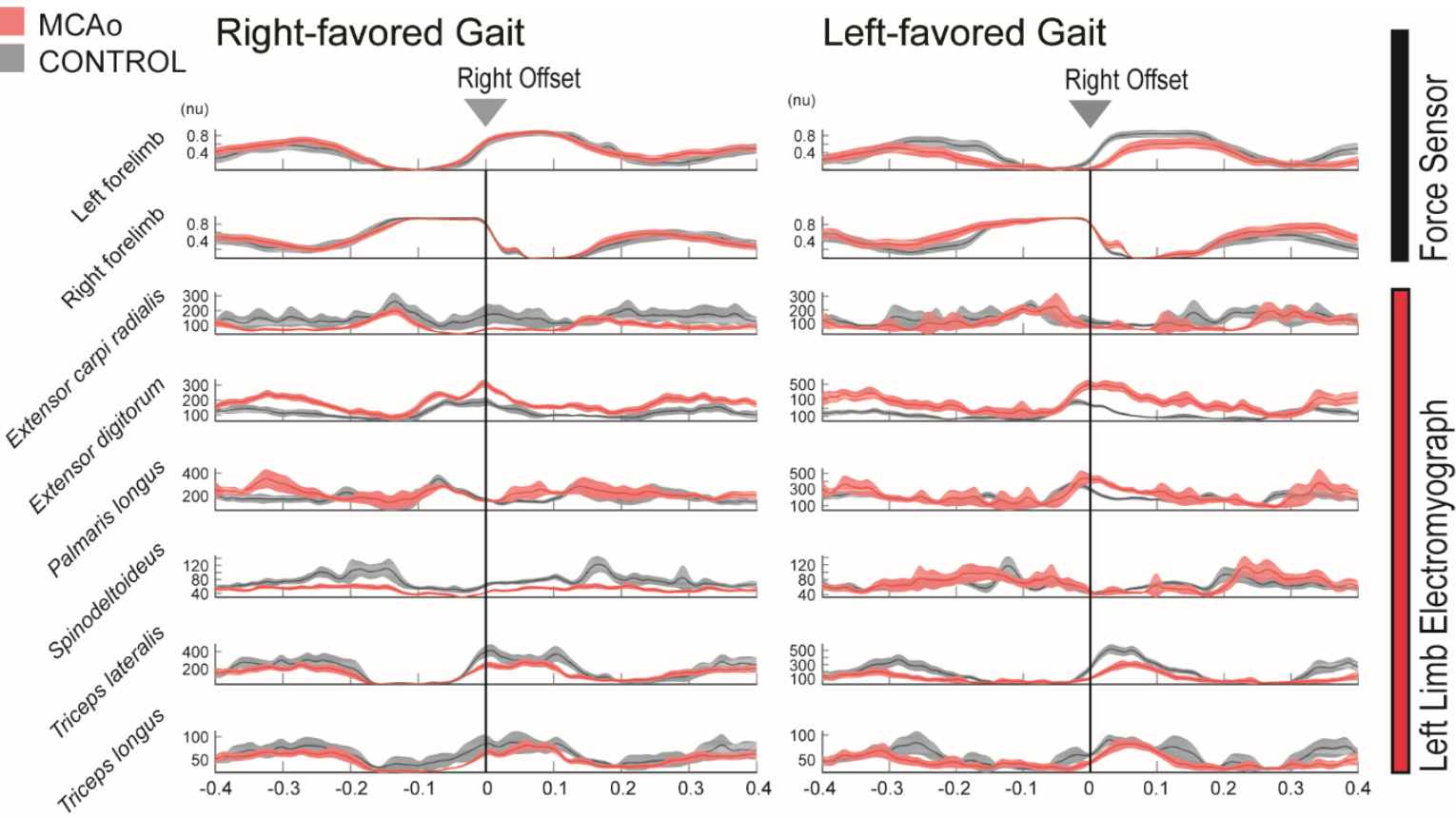

Figure 16. In vivo electromyographic recordings from the precise foot placement locomotor task. The recordings were obtained from force sensor data (top two traces) and electromyographic data from the paretic limb during in vivo locomotion on the asymmetric walkway (bottom six traces). Unilateral right-sided transient ischemic brain injury produces locomotor deficits associated with a delay of stance onset as compared to healthy lateralized locomotion. The duration of the delay is associated with prolonged spastic activity on the dorsiflexor muscle population, which ultimately contributes to the delayed onset of stance on the hemiparetic limb. The functional delay is observed predominately on the locomotor task which challenges the hemiparetic side (left-favored gait), but is not observed on the task which challenges the healthy side (right-favored gait). 
The second value used to characterize the different lateralization was the interstance index, in essence a value which analyzes the interlimb coordination during locomotion. These values produced an observable difference between the forelimb and hindlimb pairs, though no observable differences were produced between left or right forelimb-hindlimb pairs (Figure 17). There was no observable difference in the interstance phase modulation between healthy and paretic locomotion, as hemiparetic locomotion produced data of comparable significance (data not shown).

A.

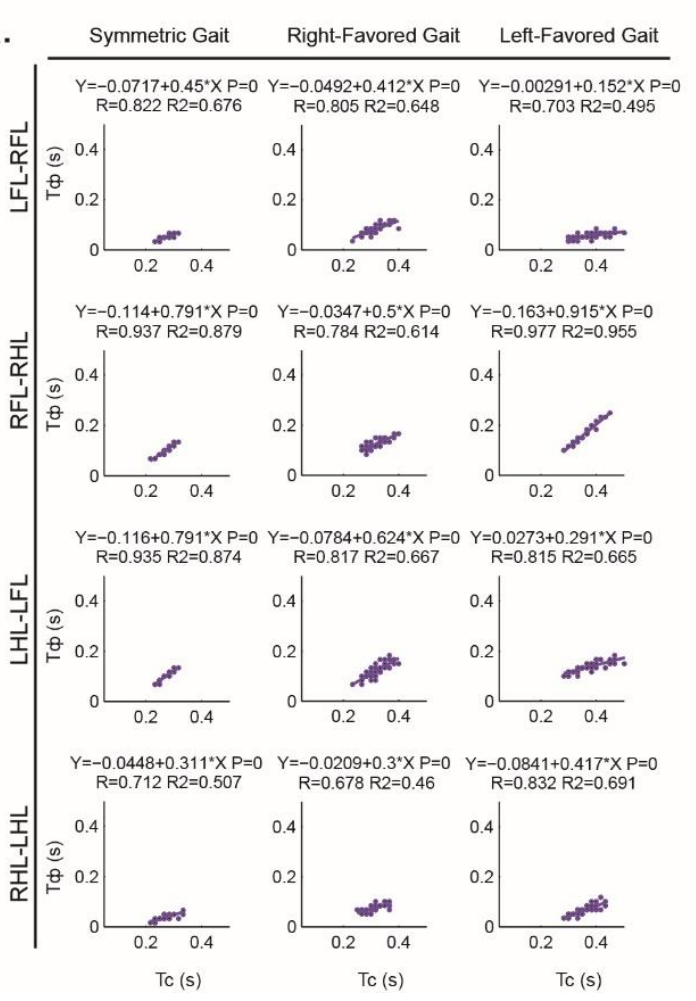

B.

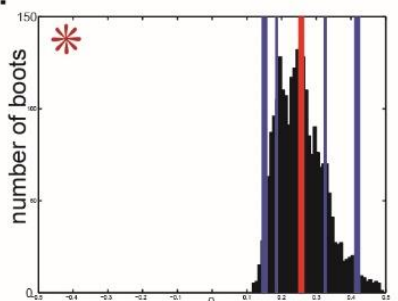

LFL-RFL |L9R6-L6R9|

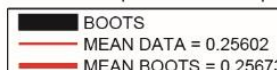
MEAN BOOTS $=0.25673$ $\mathrm{Cl}=\left[\begin{array}{ll}0.15109 & 0.41791\end{array}\right]$

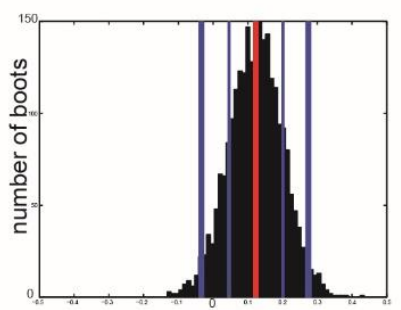

LFL-LHL |L9R6-L6R9|

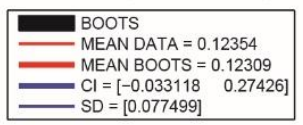

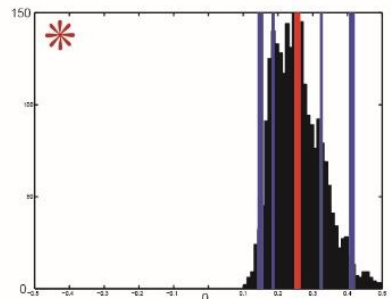

LHL-RHL |L9R6-L6R9|

BOOTS MEAN BOOTS $=0.2569$ $\mathrm{CI}=\left[\begin{array}{lll}0.14995 & 0.41281\end{array}\right]$ $\mathrm{SD}=[0.069393]$

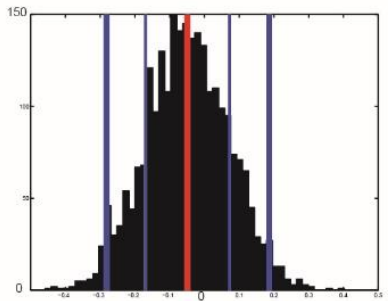

RFL-RHL |L9R6-L6R9|

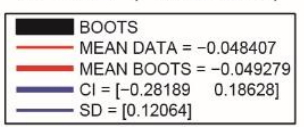

Figure 17. Intralimb coordination. A. Modulation of interstance time for the characterization of quadrupedal gait. The interstance period is defined as the period from the onset of left limb stance, to the onset of right limb stance. The duration of the interstance interval can be plotted as a function of the cycle time on the reference limb. LFL - left forelimb, RFL - right forelimb, LHL - left hindlimb, RHL - right hindlimb. B. Bootstrap analysis of interstance phase differences between left-favored and right-favored gait. Slope differences between the two lateralized conditions are statistically significant between left and right limb pairs, while the difference between the forelimb and hindlimb pairs on either side is insignificantly different. ( $\mathrm{n}=12$ subjects). 


\subsection{Mathematical modeling predicts impairments to the central drives governing flexor function}

A specifically designed strategy for analyzing the solution sets obtained from the various models was designed in order to compare the difference of parametric weight redistribution between healthy and hemiplegic solutions of the ODE (Figure 11A), as well as the solution sets that lie closest to a readapted locomotor strategy (Figure 11B). This novel, readapted solution, hypothetically represents the healthy-output solution space located most proximally to the hemiplegic-output solution space.

All model architectures were able to reproduce symmetric, as well as asymmetric locomotor behaviors (Figure 18A and 18B). In most cases, the model was able to predict the empirical targets obtained from locomotor data from healthy as well as animals which had undergone the transient middle cerebral artery occlusion procedure at a short initial range of locomotion. In some cases, the empirical targets were reached, but the duration of the cycle times on both limbs for each step was unequal, which was a criterion for data exclusion. For the simulations of healthy as well as paretic locomotion, the simulations optimized at a short cycle range were capable of reproducing nearly optimal results even at longer ranges of cycle times. Locomotor behavior from one subject, which was not reproduced properly by the model, was excluded from evaluation. 
A.

B.
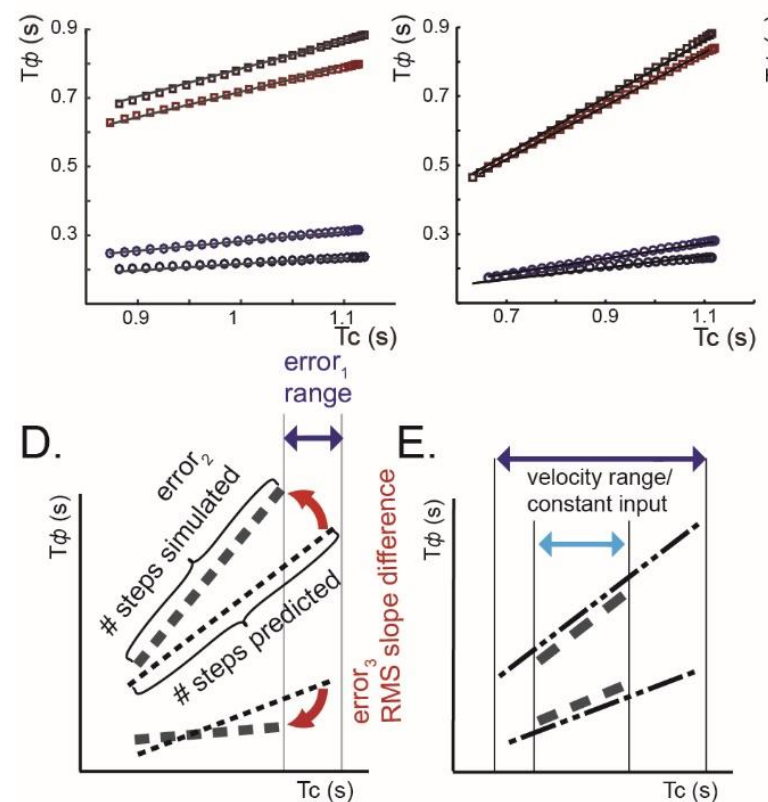

E.

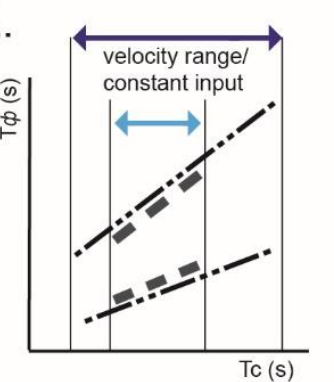

C.

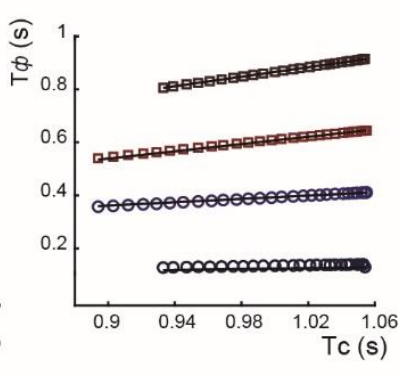

$\mathrm{y}_{\text {stance }}=\mathrm{B} 1+\mathrm{B} 2{ }^{*} \mathrm{X}$

$\mathrm{y}_{\text {swing }}=-\mathrm{B} 1+(1-\mathrm{B} 2)^{*} \mathrm{x}$

$\square$ Simulated Left Stance

Simulated Right Stance

S Simulated Left Swing

0 Simulated Right Swing

- Empirical Target

Figure 18. Output of simulated locomotor behavior. The proposed optimization scheme from Figure 12 can reproduce healthy symmetric (A.), healthy asymmetric (B.), as well as paretic asymmetric locomotion (C.). D. Schematic of the parameters taken into the compound error function comprised of a range error (error 1), output of step number predicted by the step cycle time on each limb (error 2), and the root-meansquare (RMS) error of the model output from empirical stance and swing phase regressions (error 3 ). Legend - bottom, right. The equations below demonstrate the mutual relationship of the stance and swing phases in relation to the duration of the locomotor cycle.

The process of optimization was monitored for error reduction through the MATLAB built-in fminsearch function, which reports the error value for each iteration. The error value was then plotted against the iteration count to reveal some general characteristics about the navigation process through multiparametric state-space (Figure 19). The results reveal that optimizing different model architectures is not affected by subject behaviors, but rather by the interconnectivity architecture of the particular model. Generally speaking, the optimization process for the 12 parameter models (Sherrington-type all extrinsic - Figure 19A, and Browntype all intrinsic - Figure 19B) reached half-maximum error within the first 100 iterations, and reached a logarithmic reduction within the first 200 iterations. The expanded 20 parameter models were comparatively slower to reach the half-maximum point within 150 iterations, and a logarithmic reduction within the first 250 iterations for the flexor-biased initial conditions (ICF, Figure 19D) and within the first 600 iterations for the symmetric initial conditions (ICS , Figure 
19C). The maximum rate of optimization for the 12 parameter models was between 8 and 14 and 10 percent per iteration around the $45^{\text {th }}$ iteration step, for the Sherrington and Brown types respectively, and as revealed by the first-derivative analysis of the optimization processes (Figure 19A and Figure 20B). For the 20 parameter models, the peak optimization was reached around the $100^{\text {th }}$ iteration with a maximum rate of 14 and 16 percent per iteration, for $I C^{S}$ and $I C^{F}$ initial condition sets respectively (Figure 20B and Figure 20C). Furthermore, optimizations from the $I C^{F}$ initial conditions from averaged cost reduction of 6 different subject datasets were faster at reaching the optimization minimum at 500 iterations, than the ones from $\mathrm{IC}^{s}$ initial conditions, which reached the optimization minimum within 1000 iterations (Figure 21).

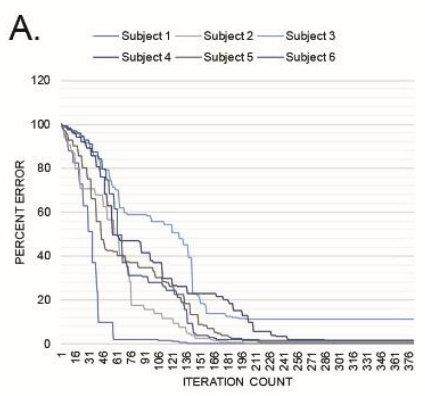

C.

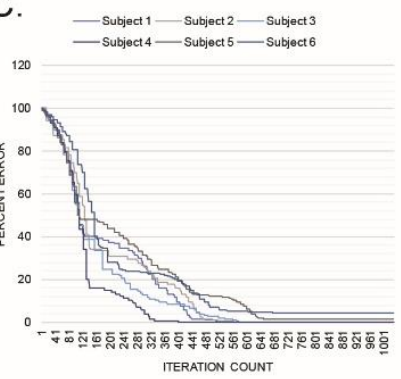

B.
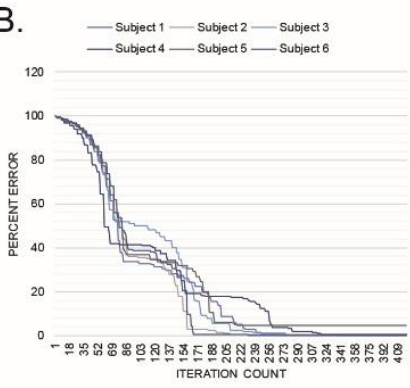

D.

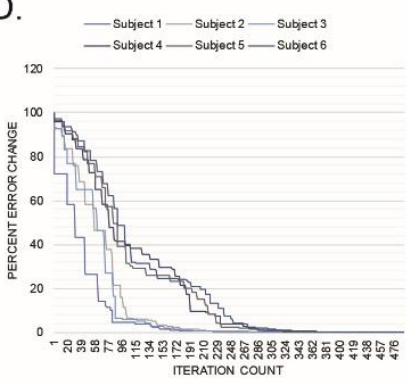

Figure 19. Cost reduction by the NelderMead method. The cost function associated with stance and swing phase divergence evaluated as the root-mean-square difference of the empirical target and the simulated output for (A.) a 12 parameter Sherrington-type allextrinsic model, (B.) a 12 parameter Browntype all-intrinsic model, (C.) an expanded 20 parameter combined model optimized from absolutely symmetric initial conditions (IC ${ }^{S}$ Equation 8) and an expanded 20 parameter combined model optimized from flexor-biased initial conditions (ICF - Equation 9) (D.). The error values were normalized for the behavior of 6 individual subjects, and plotted against the iteration count of the Nelder-Mead Simplex function. 
Figure 20. First derivative of normalized cost reduction behavior. The percent error change between each iteration was plotted against the iteration number for (A.) a 12 parameter Sherrington-type all-extrinsic model, (B.) a 12 parameter Brown-type allintrinsic model, (C.) an expanded 20 parameter combined model optimized from absolutely symmetric initial conditions (IC ${ }^{S}$ Equation 8) and an expanded 20 parameter combined model optimized from flexorbiased initial conditions (ICF - Equation 9).
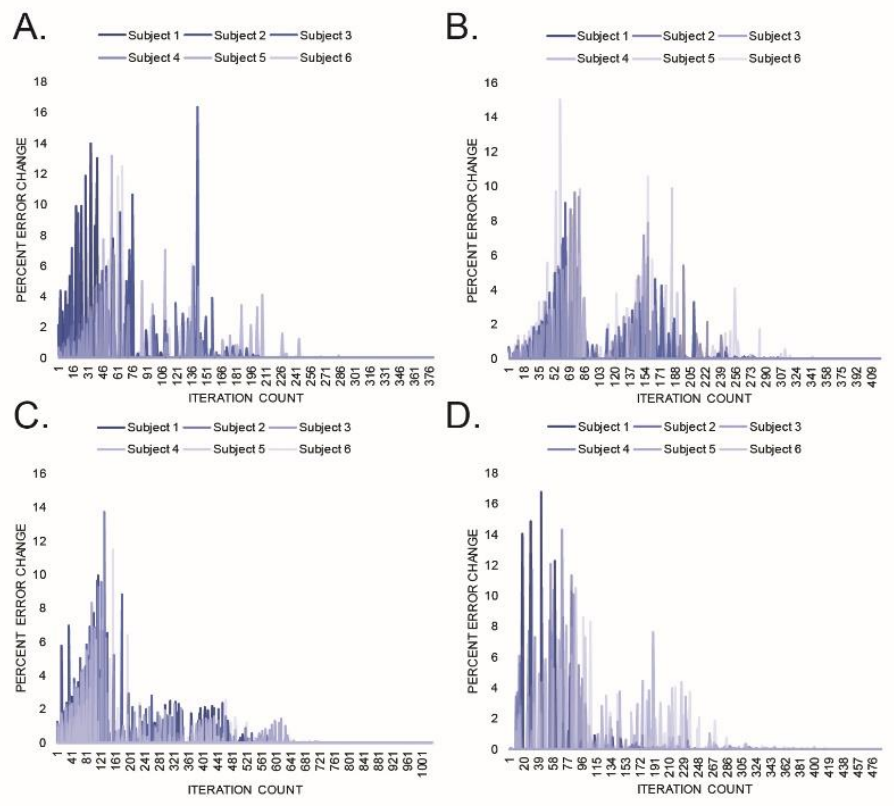

The first derivative of cost reduction also reveals that the optimization process from symmetric ICs produces three extra peaks of cost reduction rate, one in the proximity of iteration step 280 , a second in the proximity of iteration step 430, and a third in the proximity of iteration step 600. The flexor-biased initial conditions, on the other hand, only produce one extra peak cost reduction at approximately the $200^{\text {th }}$ iteration step. Together these results indicate that the trajectory of optimizing the expanded 20 parameter model is reliant on the initial position in task space. Even though the solutions sets obtained from all of the combined models (Figure 8B and $\mathbf{8 C}$ ). did not show statistical significance across all parameters (Figure 22), the flexor-dependent parameters showed significant alteration between healthy and paretic locomotion for all combined models (Figure 23A, B, G and H). Moreover, both combined models (Figures 23A and B) demonstrated a trend for significant divergence of the extrinsic parameters between healthy and paretic locomotion, and were biased towards drives regulating flexor activity. The Brown-type (all intrinsic, Figure 23C) and the Sherrington-type model (all extrinsic) were not predicted to have different modes of driving healthy lateralized gait when subjected to parametric subset analysis (Figure 
22C and D), but were predicted to preferentially involve alterations of the self-sustainment parameters in paretic locomotion (Figure 23C, D, G and H).

A.

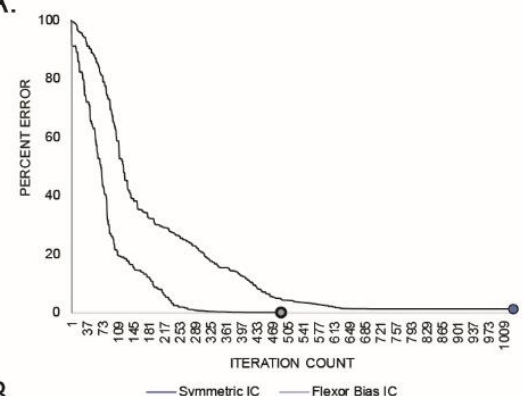

B.

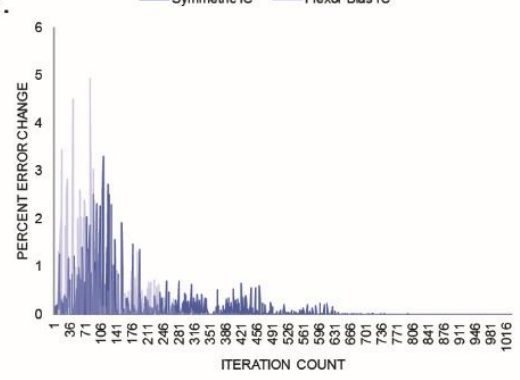

Figure 21. Different initial conditions take different paths through the task-space of cost optimization. A. Averaged behavior from optimization processes for 6 individual subjects plotted against the iteration count. The circle indicates the termination point of the optimization process. B. First derivative of normalized error behavior plotted against the iteration count. In general, symmetric initial conditions require a longer optimization process in order to converge on a solution with minimum error, converges more slowly on the solution, and utilizes smaller percent error change increments in order to achieve cost minimization.
Figure 22. Bootstrap analysis of solution sets reproducing healthy locomotion. Effective size difference produced by the combined parameter models from Figure 11B (A.), 11C (B.), 11D (C.), and 11E (D.) Neither model presented a significant parametric bias for reproducing left or right asymmetric locomotion. All effective size differences were produced by Equation 12 and analyzed for statistical significance at a boot number of 100,000 .
A.
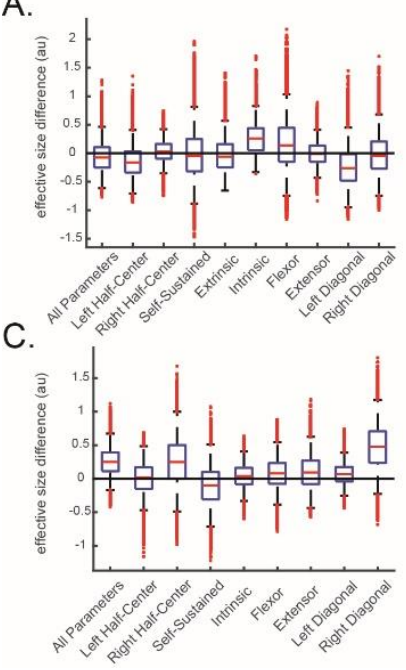

B.

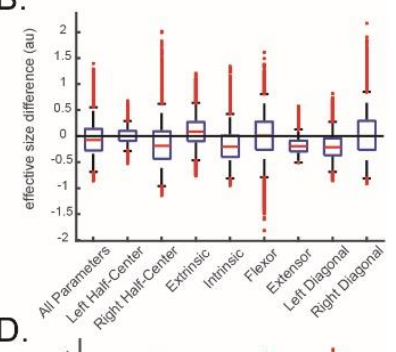

D.

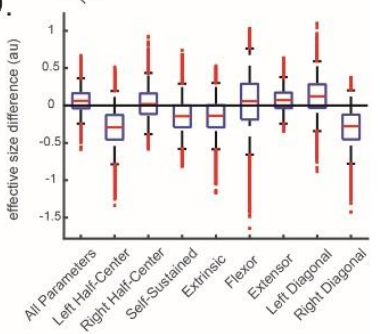


A.

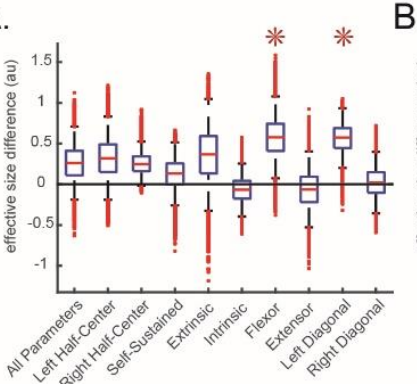

C.

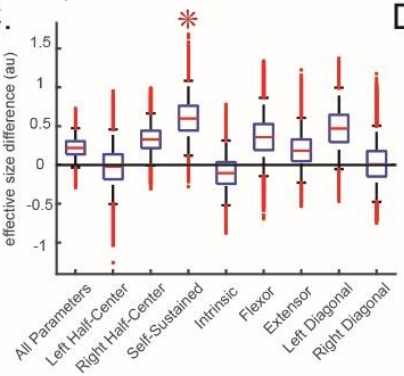

E.

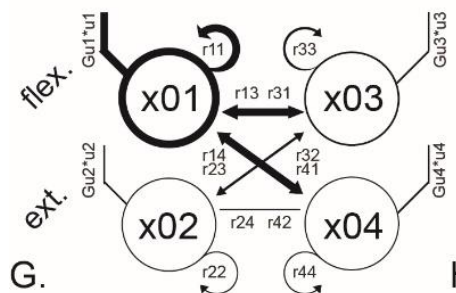

G.

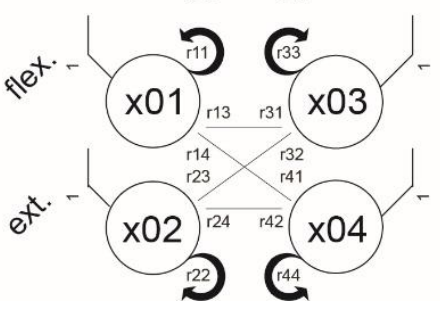

B.

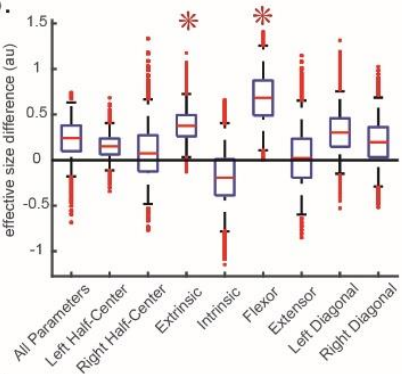

D.

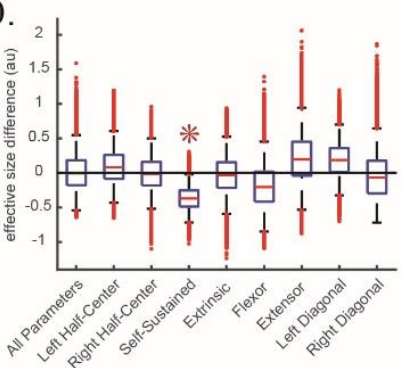

$\mathrm{F}$.
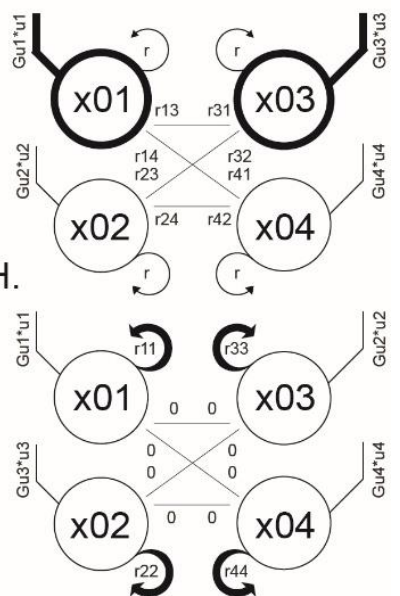

Figure 23. Analysis parametric subset weight redistribution between solution sets reproducing healthy and hemiparetic locomotion. A. The fully expanded 20 parameter model predicts greater parametric alteration of the flexor population and the left diagonal flexor-extensor coupling. B. The 17 parameter model predicts greater reliance on the extrinsic and flexor-related drives. C. and D. The fully intrinsic and fully extrinsic models predict greater reliance on the self-sustained parameters. E. The holistic model of full parametric expansion demonstrates a greater parametric difference between extrinsic and intrinsic parameters coupling the paretic flexor integrator to the contralateral extensor integrator. F. Keeping the self-sustainment parameter ' $r$ ' equivalent across all integrators shifts the parametric difference solving for healthy and paretic gait to the extrinsic flexor population, while full elimination of intrinsic connectivity in $\mathbf{H}$. predicts that paretic locomotion is obtained by maladaptation of the self-sustainment factor alone. There was no significant strategy of impairment identified in the solely intrinsic mode of coupling in G. Effective size differences were produced by Equation 12 and analyzed for statistical significance at a boot number of 100,000. $\square$ statistical significance.

\subsubsection{Hemiplegic solutions can be numerically modified to produce the output of healthy locomotion in a precise foot-placement task}

For the purpose of evaluating the parametric difference between healthy pattern reproducing solutions originating from symmetric initial conditions $\left(\mathrm{IC}^{\mathrm{SH}}\right)$ and solutions originating from flexorbiased hemiplegic initial conditions $\left(\mathrm{IC}^{\mathrm{FH}}\right)$, to those of the healthy and hemiplegic strategies obtained from IC $\mathrm{IC}^{S}$ (Equation 8) and IC $\mathrm{I}^{\mathrm{F}}$ (Equation 9) optimization results from the behavior of 6 to 8 subjects were subjected to Equation 12 to evaluate effective size variation at a boot number of 100,000 . These solutions reveal that symmetric initial conditions produce readaptation strategies 
that rely on all parametric subsets, except for the intrinsic parametric subset (Figure 24A). These ODE solutions are capable of reproducing healthy behavior reliably, but indicate that the strategy which lies closest to the hemiplegic locomotor strategy (Figure 24B) needs to rely on many parametric subsets in order to be re-optimized for healthy output. This strategy neglects to modify the intrinsic parameters, as well as the parameters contributing to extensor function. Moreover,

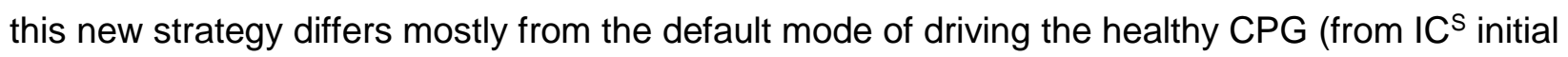
conditions, Equation 8) through self-sustainment parametric weights. Flexor-biased initial conditions $\left(\mathrm{IC}^{\mathrm{F}}\right)$ produce a hemiplegic solution which needs to be modified through all parametric subsets, except for the subset of the left half-center related parameters, self-sustainment parameters and the left diagonal parameters (Figure 24D and $\mathbf{H}$ ). This new strategy differs from the initial healthy solution through all parametric subsets, except for the self-sustainment parameters (Figure $\mathbf{2 4 C}$ and $\mathbf{G}$ ).

Solutions originating from flexor-biased initial conditions (ICF, Equation 9) differ from solutions derived from their hemiplegic IC counterpart $\left(\mathrm{IC}^{\mathrm{FH}}\right)$ mostly through neglecting the self-sustainment parametric subset to produce healthy locomotion (Figure 24C). Moreover, this solution avoids modifying the left half-center contributing parameters, as well as the left-diagonal flexor-toextensor pairings (Figure 24D). These drives are impaired most significantly in readaptation optimizations from initial conditions reproducing paretic locomotion (Figure 23A and 23E). 

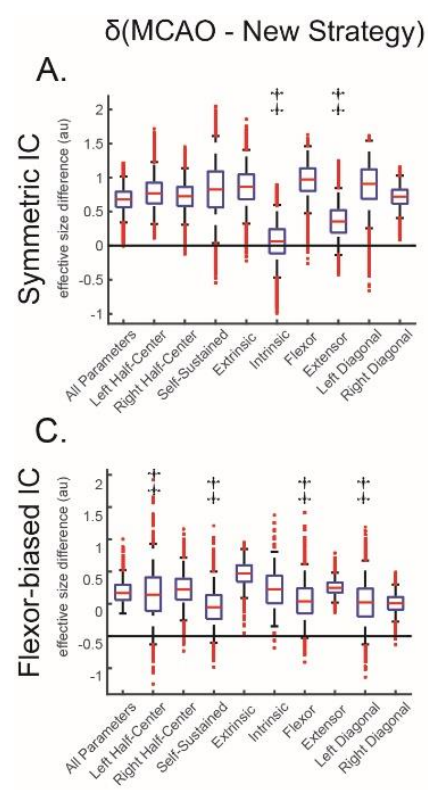

E.

D.

B

$\delta$ (Healthy - New Strategy)
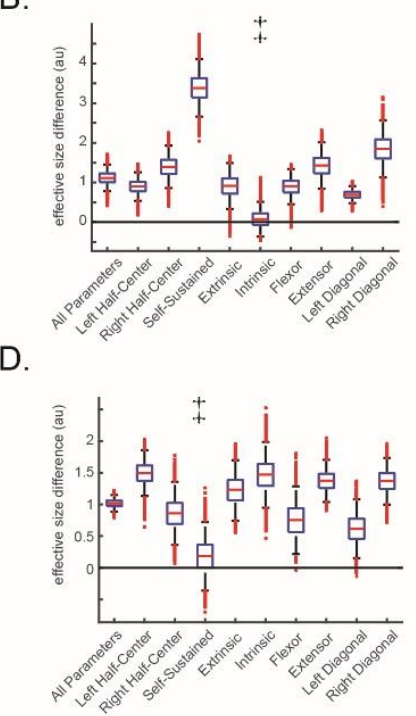

F.
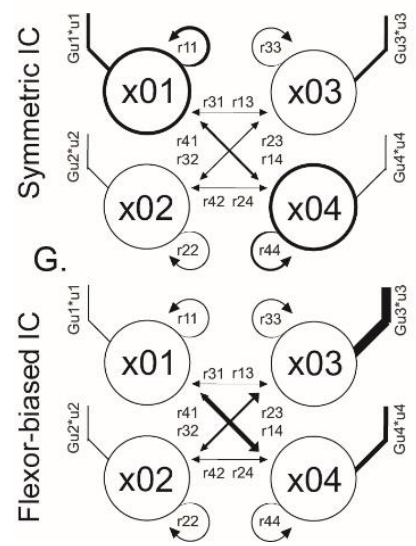

$\mathrm{H}$.
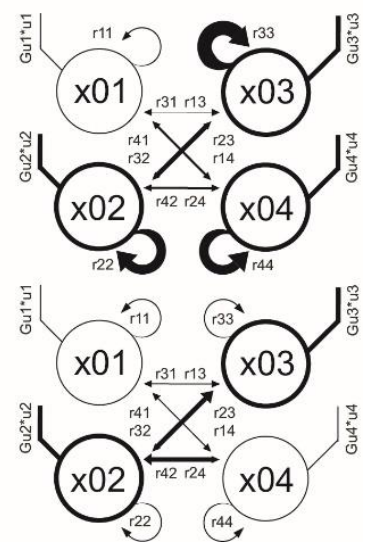

Figure 24. Statistical analysis of parametric weight distribution between readapted solutions from hemiplegic IC. A. Difference of the ICS strategy from the new strategy obtained from re-optimizing hemiplegic initial conditions $\left(\mathrm{IC}^{\mathrm{H}}\right)$. B. Difference of the hemiplegic strategy obtained from $\mathrm{IC}^{S}$ from the retrained strategy from $\mathrm{IC}^{\mathrm{HS}}$. C. Difference of the $\mathrm{IC}^{\mathrm{F}}$ strategy from the new strategy obtained from re-optimizing hemiplegic initial conditions (ICHF). D. Difference of the hemiplegic strategy obtained from $\mathrm{IC}^{\mathrm{F}}$ from the retrained strategy from $I C^{H F}$. The results from panels $\mathbf{A}$. through $\mathbf{D}$. are summarized graphically in panels E. through H. E. Difference of the ICS strategy from the new strategy obtained from reoptimizing hemiplegic initial conditions $\left(\mathrm{IC}^{\mathrm{H}}\right)$. F. Difference of the hemiplegic strategy obtained from $\mathrm{IC}^{\mathrm{S}}$ from the retrained strategy from $\mathrm{IC}^{\mathrm{HS}}$. G. Difference of the $I C^{F}$ strategy from the new strategy obtained from re-optimizing hemiplegic initial conditions (ICHF). H. Difference of the hemiplegic strategy obtained from ICF from the retrained strategy from $\mathrm{ICHF}$. Effective size differences were produced by Equation 12 and analyzed for statistical significance at a boot number of 100,000 . $\ddagger$ - statistical insignificance.

These solutions would indicate that the 'novel' (readapted) strategy would have to rely on different modes of driving the left half-center through the involvement of extrinsic and intrinsic drive modulation. Moreover, the drives of the right-to-left flexor-to-extensor coupling would have to be adjusted, specifically among the intrinsic drives, and to readapt the 'hemiparetic' mode of driving the central pattern generator. The novel CPG solution strategy is relatively indifferent to the mode of driving flexor function or the mode of driving the left-diagonal flexor-to-extensor coupling. It is also indifferent to the drives of the right half-center, as well as to the self-sustained parameters.

The general difference between $I C^{S}$ and $I C^{F}$ derived solutions of healthy locomotion is the manner in which the hemiparetic side has to be driven. These differences are distributed preferentially 
among the left half-center related parameters, the extrinsic drives, extensor related parameters, and the right-diagonal flexor-to-extensor coupling (Figure 25A). The hemiparetic solutions also demonstrate a tendency to avoid readaptation of the self-sustainment parameters, but differs from the trend of the healthy solutions by avoiding use of the right-diagonal flexor-to-extensor coupling (Figure 25B). Finally, the readapted strategies from $\mathrm{IC}^{\mathrm{SH}}$ and $\mathrm{IC}^{\mathrm{FH}}$ initial conditions are substantially more divergent from one another. These strategies employ differences in every parametric subset, with preferential weight being placed on the self-sustainment parameters (Figure 25C).

Lastly, in order to evaluate whether the readapted solutions from IC ${ }^{\mathrm{SH}}$ (Equation 10 and Figure 10 - blue trace, bottom row) and $\mathrm{IC}^{\mathrm{FH}}$ (Equation 10 and Figure 10 - orange trace, bottom row) initial conditions may be inversely close to healthy strategies from the $\mathrm{IC}^{\mathrm{S}}$ (Equation 8 and Figure 10 - orange trace, second row) and $I C^{F}$ (Equation 9 and Figure 10 - navy trace, second row) initial conditions, the readapted strategies were compared to their unlike healthy counterpart, where $I C^{S H}$ was compared to $I C^{F}$, and vice versa, IC ${ }^{F H}$ was compared to $I C^{S}$ (Figure 25D). The results reveal that the hemiparetic output derived flexor-biased $I C^{F H}$ solution is different from the symmetrically obtained $\mathrm{IC}^{S}$ solution mostly through the involvement of extrinsic and intrinsic factors (Figure 25D, left). In contrast, the hemiparetic output derived symmetric $\mathrm{IC}^{\mathrm{SH}}$ solution is different from the flexor-bias obtained $\mathrm{IC}^{\mathrm{FH}}$ solution through the involvement of the self-sustained factor predominately (Figure 25D, middle). Finally, the difference between these readapted solutions is a result of the additive divergence between all parametric subsets, and reveals that the solution space obtained from the different pairs of initial conditions is substantially different, even when compared to the solution space producing either healthy or hemiparetic locomotion. 


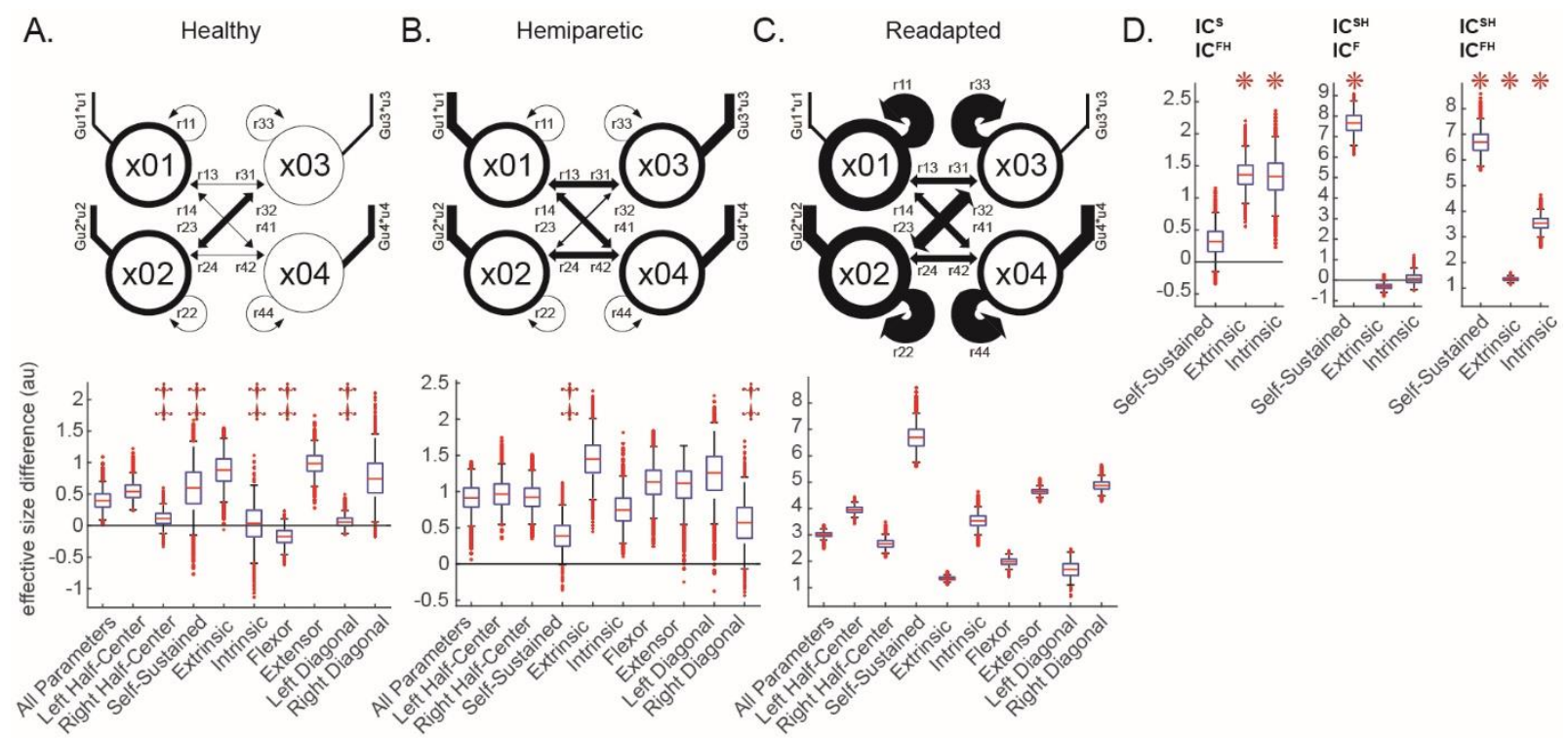

Figure 25. Parametric subset weight distribution differences between $\mathrm{IC}^{\mathrm{S}}$ and $\mathrm{IC}^{\mathrm{F}}$ solutions. $A$. The CPG driving strategy is significantly different between solutions originating from symmetric and flexorbiased initial conditions, and is mostly reliant on differences involving the left half-center, as well as rightdiagonal flexor-extensor coupling. The manner of driving is not different among the right half-center, intrinsic, or left-diagonal parametric subsets. B. The solutions of hemiparetic gait from IC $\mathrm{IC}^{S}$ and $I \mathrm{IC}^{\mathrm{F}}$ initial conditions differ in almost every parametric subset, except for the self-sustained parameters, and the rightdiagonal parameters. C. Solutions from (B.) were used as initial conditions to produce a readapted locomotor strategy, and the parametric subsets of the retrained solutions were analyzed for divergence using the Bootstrap method. The data reveals that the readapted strategy differs most significantly from the healthy and hemiparetic solutions obtained from IC $\mathrm{C}^{S}$ and $I \mathrm{C}^{\mathrm{F}}$ through the employment of readaptation along the self-sustainment factor. D. Readapted non-hemiplegic solutions of the ODE differ from each other along combinations of self-sustained, extrinsic and intrinsic parametric subsets. IC ${ }^{s}$ - solutions from symmetric initial conditions; ICF - solutions from flexor-biased initial conditions; ICHS - solutions from symmetrically obtained hemiplegic solutions; ICHF - solutions from flexor-bias obtained hemiplegic solutions. All effective size differences were produced by Equation 5 and analyzed for statistical significance at a boot number of 100,000. $\square$ statistical significance; $\ddagger$ - statistical insignificance.

\subsection{Solutions of different intrinsic parameter contribution predict different modes of readaptation}

Solutions which employ $30 \%, 60 \%$ or $220 \%$ intrinsic parameter contribution relative to extrinsic parameter contribution were also able to predict a trend of parametric subset divergence between solution sets producing healthy (Figure 26A, left column) and hemiparetic (Figure 26A, middle column) rhythmic patterns (Figure 26B, left column). At the same time, the difference between the healthy and the hemiparetic strategy was not found to be significantly different among parametric subsets as defined in Table 2. 
From individual parametric divergence analysis, the solution which predicts $1 \%$ contribution of the intrinsic parameters predicted impairments to the modulatory gain of input ' $u$ ' ('Gu') to the hemiparetic flexor population as the key factor involved in the production of hemiparetic output. In order to rehabilitate this strategy, the tertiary optimization process (Figure 11B, 'Optimization III) suggests that readapting the solution through modifications of inputs to the non-hemiparetic flexor and extensor may be employed as a viable strategy, which ultimately modulates the rhythmicity of the hemiparetic half-center through various self-sustained and intrinsic couplings.

When statistical analysis was employed on parametric subsets (Table 2), there was no significant difference identified between the healthy and hemiparetic strategy for the $30 \%, 60 \%$, and $220 \%$ solutions (data not shown). When statistical analysis was performed on individual parameter divergence, single parameter associations (Figure 26B, first column, and Figure S2) were identified as the source of the altered rhythmicity. These results would indicate that in these solutions to the ordinary differential equation, significantly large perturbations to a small number of parameters was capable of reproducing either healthy or hemiparetic locomotor output, without a single particular trend of parametric subset modification.

Similarly to solutions which diverge in their symmetry within given intrinsic connectivity state, solutions which output healthy locomotor strategies at higher intrinsic parameter contributions (Figure 10 - third row) demonstrate that the parametric ratios reproducing hemiparetic output can be modified to produce a healthy locomotor pattern through various means of perturbations (Figure 26A and B, second and third column). For the 30\% intrinsic contribution model, the difference between the solutions which predicted hemiparetic pattern output and the solutions which predicted a readapted healthy output from hemiparetic initial conditions (initial conditions summarized in Equation 10 and Figure 10, bottom row, gray trace), may rely on modifying the inputs to the right (non-hemiparetic) half-center, as well as the state-dependent intrinsic as well as self-sustainment inputs originating from the right half-center in order to modify the locomotor 
output rhythm into a healthy pattern (Figure 26B, second row). This strategy is also different from the healthy solution from symmetric initial conditions (Equation 8 and Figure 10, second row) through the involvement of contralateral flexor-flexor and extensor-extensor couplings, as well as the left-to-right extensor-flexor coupling.

The $60 \%$ intrinsic contribution model (initial conditions summarized in Equation 10 and Figure 10, bottom row, yellow trace) predicted that the solution outputting hemiplegic locomotion may be modified through modulating the extrinsic drives to the extensor rhythm generating centers, as well as the contralateral intrinsic extensor-extensor couplings, as well as the self-sustainment parameters modulating the activity of the extensor integrators (Figure 26B, third row). This strategy differs from the original healthy pattern strategy through greater modulation of the extrinsic, self-sustainment, and intrinsic parameters contributing to right-half-center rhythm, as well as the extrinsic and self-sustainment parameters contributing to left half-center rhythm.

The $220 \%$ intrinsic parameter contribution solution (initial conditions summarized Equation 10 and Figure 10, bottom row, azure trace) predicted that modification of the 'hemiparetic' parameters governing left-to-right extensor-extensor couplings, as well as the diagonally coupled hemiparetic flexor to non-hemiparetic extensor descending drives, can contribute to rhythm modification which can resemble healthy locomotor output (Figure 26B, bottom row). This novel strategy is also different from the initial healthy strategy mainly through the involvement of the extrinsic contributions to the hemiparetic extensor, as well as contralateral hemiparetic-to-healthy extensor to flexor coupling.

Interestingly, the flexor-flexor coupling was identified as a target of potential modulation in both the $60 \%$ and $220 \%$ intrinsic solutions. During healthy locomotion, these parameters are expected to be of relatively little contribution to the stability of the locomotor pattern, but theoretically may posses a high parametric weight which could contribute to rapid integration termination associated with coincident swing. This coincident swing is characteristic of a type of running gallop in 
quadrupedal animals, and inevitably occurs during high locomotor speeds. Such behavior in the empirical data was excluded from evaluation, but it is possible that the model is capable of reproducing such behavior when simulating steps of higher velocity.

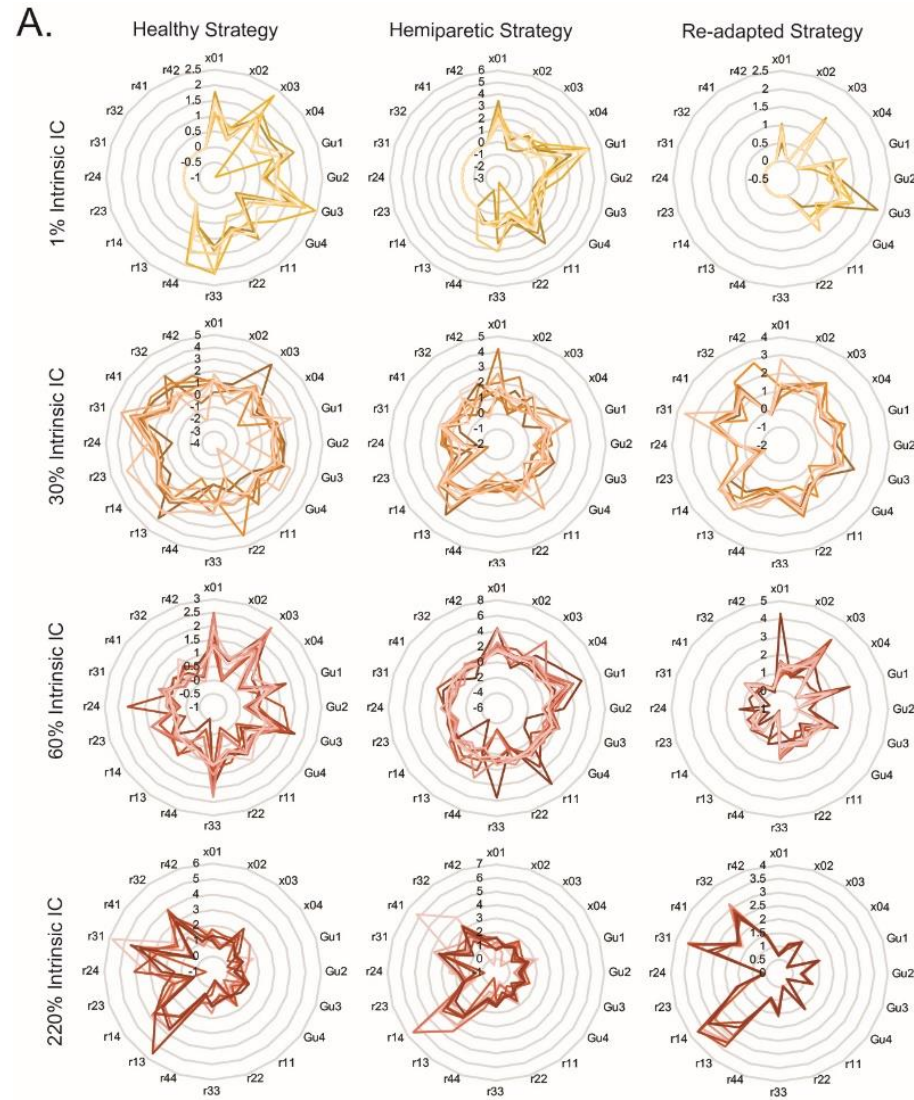

B.

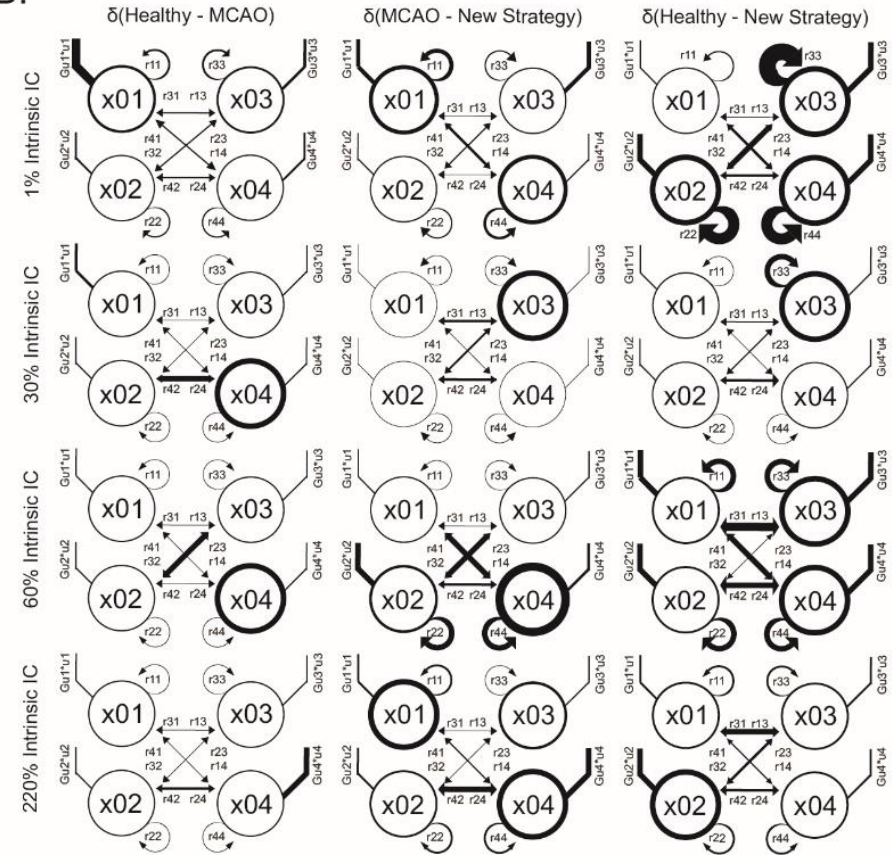

Figure 26. Solutions of different intrinsic parameter contributions have different manners of predicting left hemiparetic modifications of the central pattern generator model. A. Radar plots of the different solutions. Parametric weight distributions for solutions bearing a $1 \%$ intrinsic parameter contribution (top row), a $30 \%$ intrinsic parameter contribution (second row), a $60 \%$ intrinsic parameter contribution (third row), and a $220 \%$ intrinsic parameter contribution. The healthy strategy (left column), hemiparetic strategy (middle column), and the readapted strategy from initial conditions simulating hemiparetic locomotion (see Equation 10, right column) bear statistically significant trends of alteration. B. Graphical representation of parametric alteration trends from single parameter analysis. Effective size distribution was analyzed for significance by the Bootstrap method from healthy to hemiparetic solutions (left column), hemiparetic to readapted solutions (middle column), and healthy to readapted solutions (left column), and the relative effective size differences were demonstrated by line thickness of the pertaining connections. Each trace in $\mathbf{A}$. represents the solution for behaviors observed in 6 to 8 individual subjects. Effective size differences were calculated by Equation 12 between each designated solution set group indicated in individual panels. All effective size differences were evaluated for statistical significance by the Bootstrap method at a boot number of 100,000 . 


\subsection{Greater intrinsic parameter contribution is associated with greater stability}

Each parametric subset (extrinsic, self-sustained, or intrinsic) was subjected to 30, 20 or 10 signal-to-noise ratio by using Equation 11 and the MATLAB built-in awgn function, where a greater ratio number is indicative of greater signal-to-noise contribution (less noise) for a total number of 15 times (Figure 27), and these perturbed parametric sets were used to evaluate the stability of the output through the same error function used for optimizations (Figure 18D). The results (Figure 28) indicate that the different solutions to the 20 parameter models have different stability properties.

First, even under low levels of extrinsic noise (30 SNR) the $1 \%$ intrinsic solution was not capable of maintaining a low error rate (Figure 28A, '1\% intrinsic'), with an approximately 100 -fold error increase from the optimized solution. The logarithmic error increase was comparable for intermediate 20 SNR extrinsic parameter noise, with an approximately 125 -fold error increase, and yet higher for the high intensity 10 SNR extrinsic parameter noise, with an approximately 2000 -fold error increase. When subjected to noise at the self-sustainment parameters, the $1 \%$ intrinsic model showed a similar tendency for instability, with a 30-fold error increase for the 30 SNR noise level, a 100-fold error increase for 20 SNR noise level, and a 300-fold increase for the 10 SNR noise level. The $1 \%$ intrinsic solution did not, however, respond in high error rates when perturbing the intrinsic parameters, which indicates that the intrinsic parameters in such a solution do not contribute greatly to the stability of the system.

The $30 \%$ intrinsic solution (Figure 28A, '30\% intrinsic') was moderately better at handling low level extrinsic parameter noise, with a 20 -fold error increase for 30 SNR noise, a 300 -fold error increase for the 20 SNR noise, and an 800-fold error increase for 10 SNR noise. When noise was introduced to the self-sustainment parameters, the $30 \%$ intrinsic solution performed comparably better than most other solutions, with a 1-to-1 error change for 30 SNR and 1-to-2 error change for 20 SNR noise. This solution performed better at high self-sustained noise intensities as well, 
with a mild 20 -fold increase in error for 10 SNR noise. When subjected to intrinsic parameter noise, this solution performed moderately well at 30 SNR, with an average of 20 -fold error change, moderately well at 20 SNR, with an average 30-fold error change, but did worse on the high 10 SNR noise perturbation with a 600 -fold average error change.

Under extrinsic parameter perturbation, the $60 \%$ intrinsic parameter contribution solution (Figure 28A, ' $60 \%$ intrinsic') responded with an average 10-fold increase at low noise intensities of 30 SNR, an average of 60 -fold error increase at intermediate 20 SNR, and an average of 2000 -fold error increase at high noise levels of 10 SNR. When noise was applied to the self-sustained parameters, this solution performed comparatively worse than the $30 \%$ intrinsic solution at low (3-fold error increase at 30 SNR), moderate (10-fold error increase at 20 SNR), and high noise levels (30-fold error increase at 10 SNR). The $60 \%$ intrinsic solution performed comparatively better than the $30 \%$ intrinsic solution when subjected to intrinsic parameter noise at low (10-fold error increase at 30 SNR), moderate (15 fold error increase at 20 SNR), and high noise levels (500-fold error increase at 10 SNR).

Ultimately, the $220 \%$ intrinsic solution (Figure 28A, '220\% intrinsic') outperformed all models at all levels of extrinsic and self-sustained parameter noise, but performed the worst at low and intermediate intrinsic parameter noise. This solution tended to remain stable, with a 1-to-1 error change from optimum at low intensity 30 SNR extrinsic parameter noise, and a 3-fold error increase at moderate 20 SNR extrinsic parameter noise, though a comparative 300 -fold error increase was detected at high intensity noise levels of 10 SNR. At low levels of self-sustained parameter noise, the $220 \%$ intrinsic solution had a tendency to halve, even decimate the error, as these small perturbations were likely to find other slightly more optimal solution points, even though the original error rate of the initial solution was significantly small. At moderate 20 SNR self-sustained parameter noise, this solution performed better than all other solutions with a 1-to1 error change. This solution outperformed the other solute sets even at high 10 SNR noise levels 
to the self-sustained parameters, with a modest 5-fold increase. When noise was introduced to the intrinsic parameters, the $220 \%$ intrinsic solution responded with a large 300 -fold increase to low level 30 SNR noise, a similar 400-fold error increase under moderate 20 SNR noise, and a 500-fold increase under high 10 SNR noise levels.

All four solution sets had a similar percent velocity response to standardized input in arbitrary units, and diverged within 5\% of the identity trend (Figure 28B through E), though they were all significantly different in their initial (Figure 28F) and final velocities (Figure 28H) under equivalent input ' $u$ ' in arbitrary units. The $1 \%$ solution output produced the lowest initial velocity of $13 \mathrm{~cm} / \mathrm{s}$ and lowest maximal velocity of $20 \mathrm{~cm} / \mathrm{s}$. The $30 \%$ solution output produced a low velocity of 32 $\mathrm{cm} / \mathrm{s}$ and a maximum velocity of $47 \mathrm{~cm} / \mathrm{s}$. The $60 \%$ solution produced a $20 \mathrm{~cm} / \mathrm{s}$ minimum velocity and a maximum velocity of $30 \mathrm{~cm} / \mathrm{s}$. Finally, the $220 \%$ solution produced a minimal velocity of 40 $\mathrm{cm} / \mathrm{s}$ and a maximal velocity of $60 \mathrm{~cm} / \mathrm{s}$. All of these locomotor ranges are within the reported locomotor speeds for mice and rats from other literature ${ }^{241}$, as well as this study (data not shown). Finally, the ratio of number of steps per second to linear input unit was not changed between the minimum and maximum stepping speeds for the $1 \%$ intrinsic solution (remained at 2 steps $^{*} \mathrm{~s}^{-1} \mathrm{au}^{-}$ 1) and $60 \%$ solution (remained at 3 steps $^{*} \mathrm{~s}^{-1} \mathrm{au}^{-1}$ ), while it decreased for the $30 \%$ intrinsic solution ( 5 to 3.3 steps $^{*} \mathrm{~s}^{-1} \mathrm{au}^{-1}$ for minimum and maximum rate of stepping), and the $220 \%$ intrinsic solution (6 to 3.83 steps $^{\star} \mathrm{s}^{-1} \mathrm{au}^{-1}$, Figure $28 \mathrm{G}$ and I).

Under maximal input ' $u$ ', the $1 \%$ intrinsic solution produced a velocity change of $33 \%$, with a range of $28 \%$ to $35 \%$. The $30 \%$ intrinsic solution produced a velocity change of $30 \%$, with a range of $9 \%$ to $49 \%$ maximal velocity. The $60 \%$ solution had an average of $32 \%$ from maximum velocity, with a range of $13 \%$ to $48 \%$ maximal velocity. Finally, the $220 \%$ solution averaged at $31 \%$ maximal velocity, with a range of $7 \%$ to $43 \%$ maximal velocity (Figure $28 \mathbf{J}$ ). Under normalized input ' $u$ ', these velocity changes corresponded to $8.1 \mathrm{~cm}^{*} \mathrm{~s}^{-2} \mathrm{au}^{-1}$, for the $1 \%$ intrinsic model, $14.9 \mathrm{~cm}^{*} \mathrm{~s}^{-2} \mathrm{au}^{-}$ 
${ }^{1}$ for the $30 \%$ intrinsic model, $14.8 \mathrm{~cm}^{*} \mathrm{~s}^{-2} \mathrm{au}^{-1}$ for the $60 \%$ intrinsic model, and $16.1 \mathrm{~cm}^{*} \mathrm{~s}^{-2} \mathrm{au}^{-1}$ for the $220 \%$ intrinsic model (Figure 28K).

A.

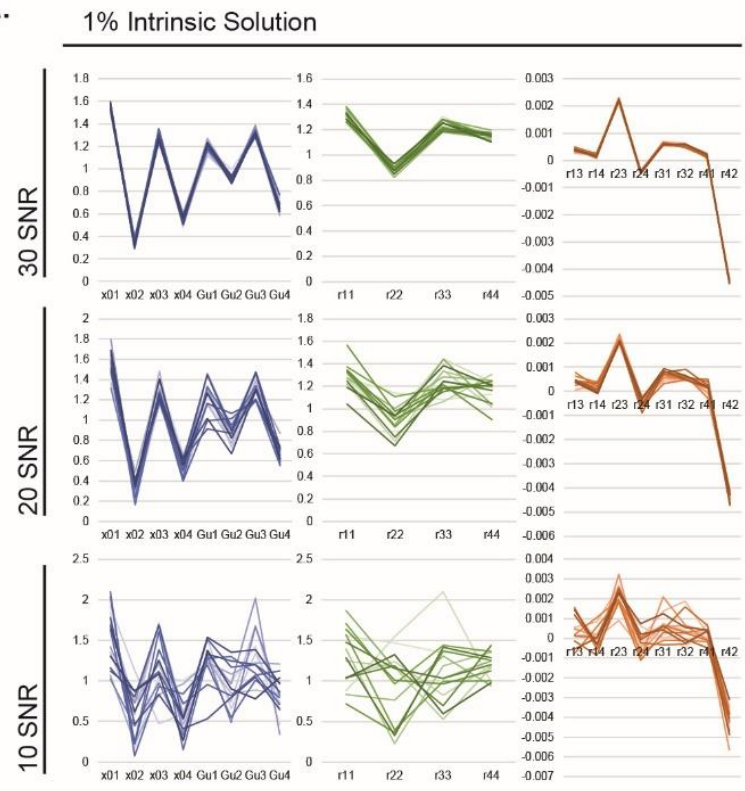

C.

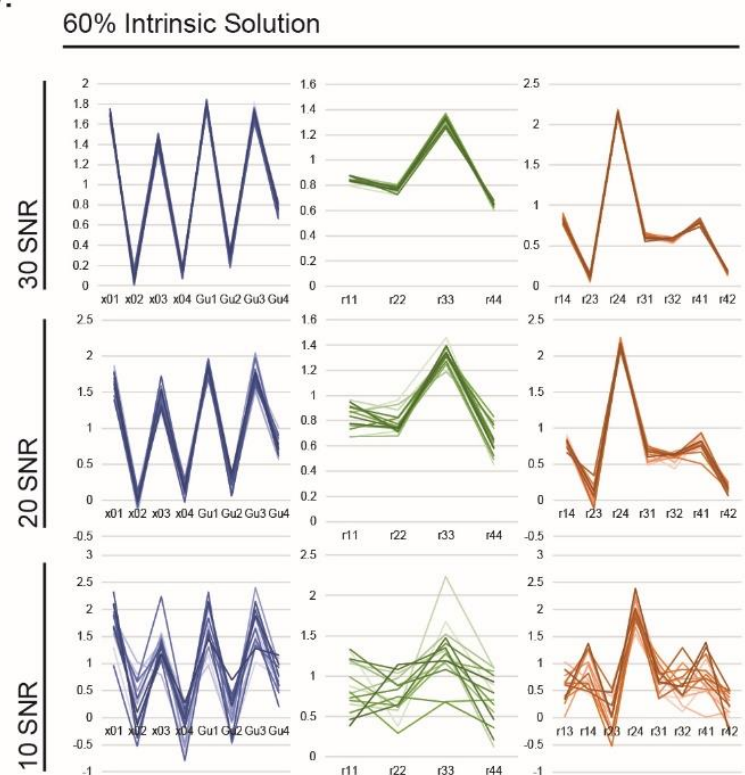

B.
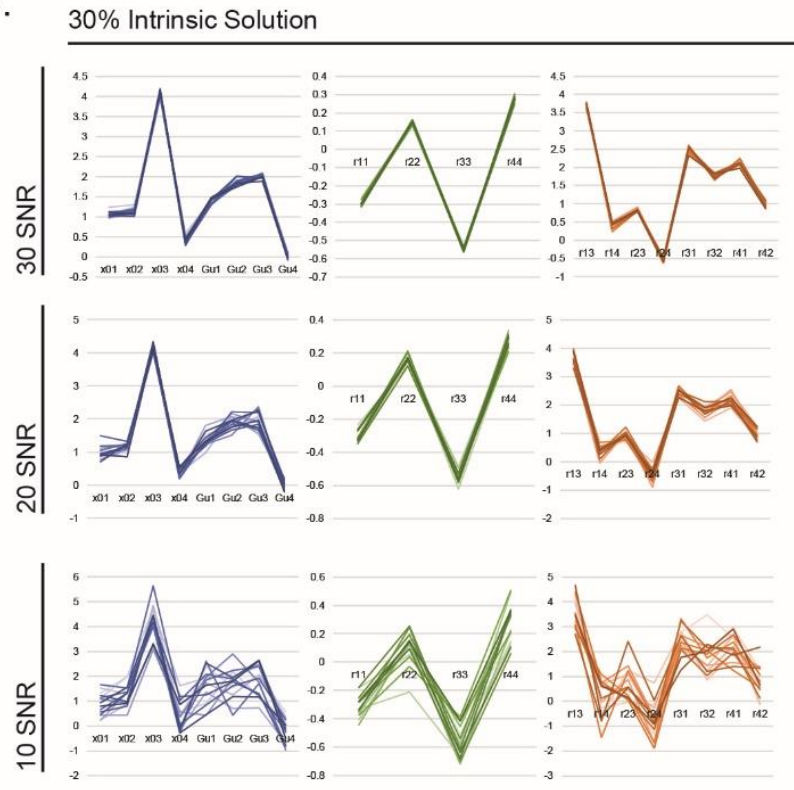

D.

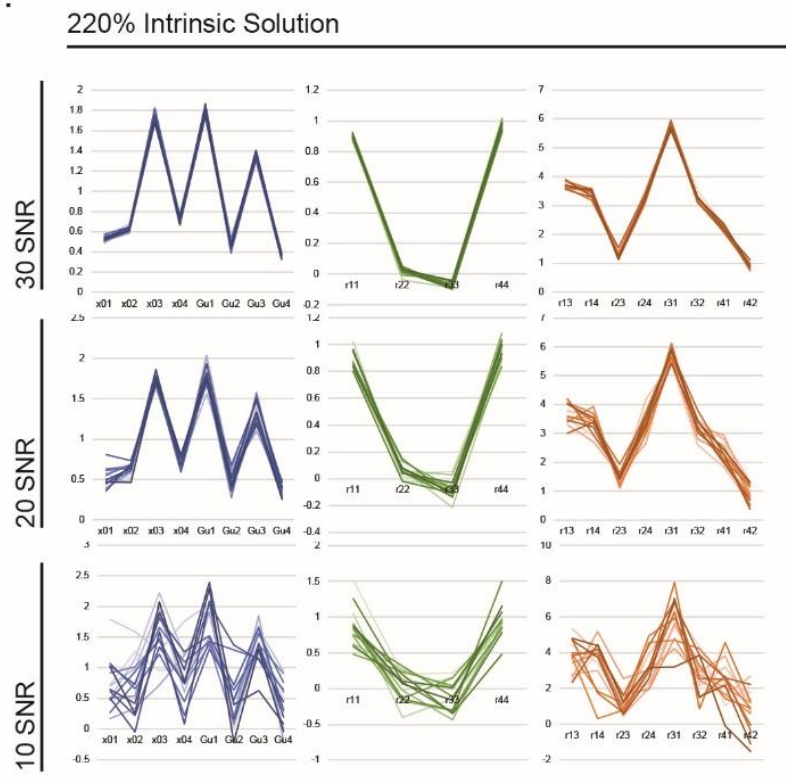

Figure 27. Introduction of noise to parametric subsets. Parametric perturbations were done by introducing noise of 30,20 , or 10 SNR to extrinsic, self-sustainment, or intrinsic parametric subsets for the most optimum $1 \%$ intrinsic contribution solution (A.), the most optimum $30 \%$ intrinsic contribution solution (B.), the most optimum $60 \%$ intrinsic contribution solution (C.), and the most optimum $220 \%$ intrinsic contribution solution (D.). Each trace is representative of a separate perturbation trial (a total of 15 trials). SNR - signal to noise ratio. Blue trace - extrinsic parameters; green trace - self-sustainment parameters; orange trace - intrinsic parameters. 


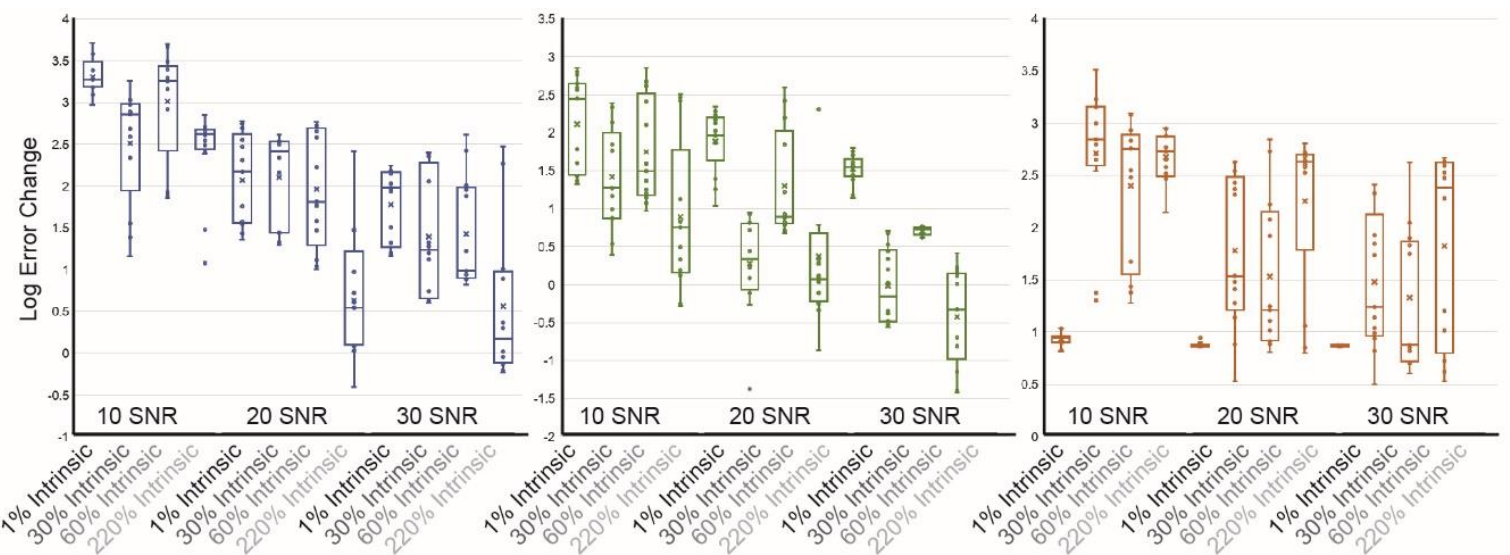

B.

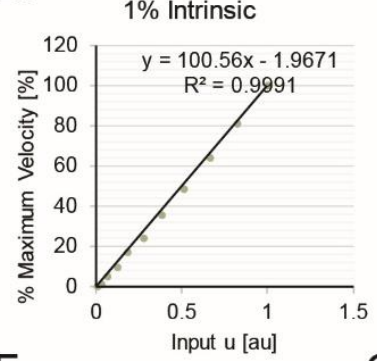

$\mathrm{F}$.
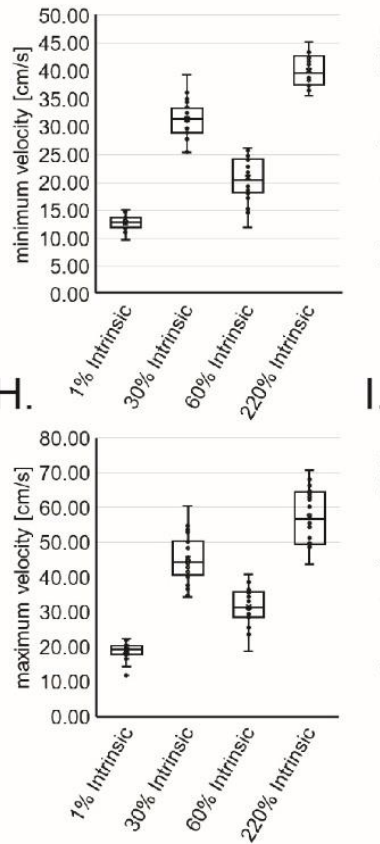

C.

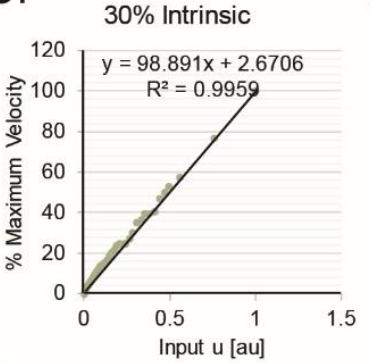

G.
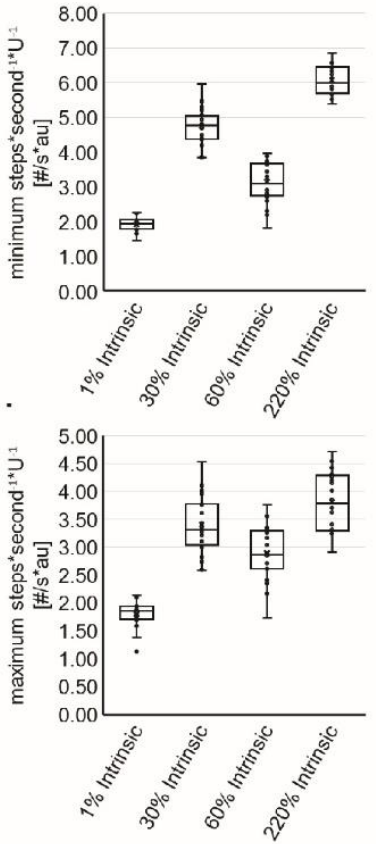

D.

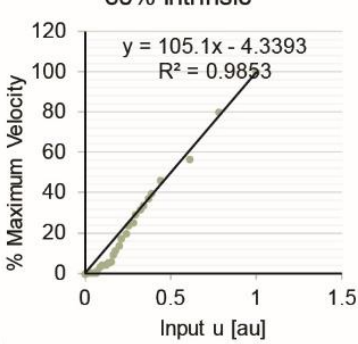

J.

E.

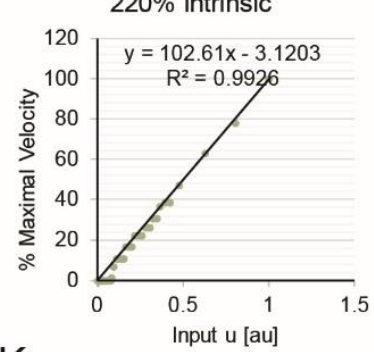

$\mathrm{K}$.

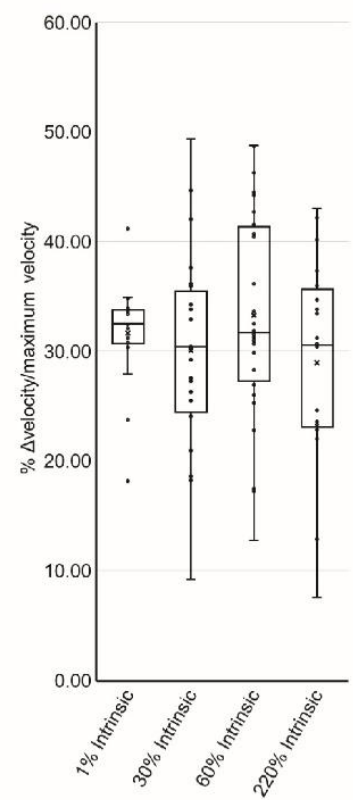

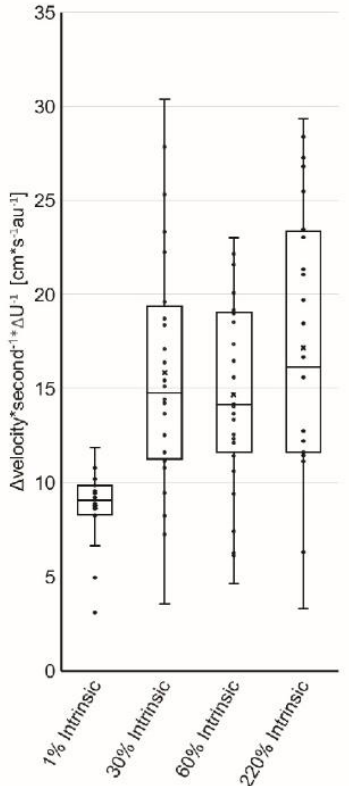

Figure 28. Stability and velocity analysis of four different healthy solutions. A. Four solutions simulating healthy locomotor output were subjected to parametric perturbations represented in Figure 27A through 27D, and analyzed for deviation from the optimized solution. B. through E., - linear regression of percent maximal simulated velocity and input ' $u$ ' in arbitrary units for the four different solutions. Black line inset - identity regression ' $P$. F. Minimum simulated velocity adjusted for a stride length of $15 \mathrm{~cm}$. G. Minimum steps per second and arbitrary input ' $u$ '. H. Maximum simulated velocity adjusted for a stride length of $15 \mathrm{~cm}$. I. Maximum steps per second and arbitrary input ' $u$ '. J. Percent velocity change from maximal velocity. $\mathbf{K}$. Velocity change per change of arbitrary input ' $u$ '. 


\subsubsection{Solutions of different output are stable along different subsets of flexor and extensor related parametric pairs}

Healthy, hemiparetic, or readapted output solutions were subjected to noise of 10 signal-to-noise ratio intensity, and the resulting output was compared against the optimized solution from each subtype. The results reveal that the solutions behave differently under perturbation stress to different pairs of flexor and extensor related parameters, and as defined in Table 3, Section

4.10.3. Generally, the solutions of healthy locomotor output tend to be more stable along the flexor integrator related parameters among all solutions of different relative intrinsic parameter contribution. This stability is somewhat lost in the solutions of hemiparetic output, and ameliorated in the solutions of readapted output. This trend is not observed among the extensor integrator related parameters, which tend to render lower resistance to perturbations among all output types.

In evaluations of healthy output, noise introduction to extrinsic flexor integrator related parameters ' $x 01$ ', ' $x 03$ ', 'Gu1', and 'Gu3' produced a 50 -fold error change in the $1 \%$ intrinsic parameter contribution solution, a 170 -fold error change in the $30 \%$ intrinsic parameter contribution solution, a 310 -fold error change in the $60 \%$ intrinsic parameter contribution solution, and a 170 -fold error change in the $220 \%$ intrinsic parameter contribution solution (Figure 29A, left orange boxplots). For the hemiplegic output, noise introduction to the same parameters produced a 1000 -fold error change in the $1 \%$ intrinsic parameter contribution solution, a 125 -fold error change in the $30 \%$ intrinsic parameter contribution solution, a 560 -fold error change in the $60 \%$ intrinsic parameter contribution solution, and a 400 -fold error change in the $220 \%$ intrinsic parameter contribution solution (Figure 29A, left red boxplots). In the solution sets reproducing readapted output from hemiplegic initial conditions, the introduction of 10 SNR noise resulted in 150-fold error change in the $1 \%$ intrinsic parameter contribution solution, a 15 -fold error change in the $30 \%$ intrinsic parameter contribution solution, a 35 -fold error change in the $60 \%$ intrinsic parameter contribution 
solution, and a 4.4 -fold error change in the $220 \%$ intrinsic parameter contribution solution (Figure 29A, left blue boxplots).

Likewise, in evaluations of healthy output, noise introduction to extrinsic extensor integrator related parameters ' $x 02$ ', ' $x 04$ ', 'Gu2', and 'Gu4' produced an average of 1800 -fold error change in the $1 \%$ intrinsic parameter contribution solution, a 350 -fold error change in the $30 \%$ intrinsic parameter contribution solution, a 180 -fold error change in the $60 \%$ intrinsic parameter contribution solution, and a 300 -fold error change in the $220 \%$ intrinsic parameter contribution solution (Figure 29A, right orange boxplots). For the hemiplegic output, noise introduction to the same parameters produced an averaged 1000-fold error change in the $1 \%$ intrinsic parameter contribution solution, an averaged 450 -fold error change in the $30 \%$ intrinsic parameter contribution solution, a 400 -fold error change in the $60 \%$ intrinsic parameter contribution solution, and a 160 -fold error change in the $220 \%$ intrinsic parameter contribution solution (Figure 29A, left red boxplots). In the solution sets reproducing readapted output from hemiplegic initial conditions, the introduction of 10 SNR noise resulted in 2500 -fold error change in the $1 \%$ intrinsic parameter contribution solution, a 500 -fold error change in the $30 \%$ intrinsic parameter contribution solution, a 500 -fold error change in the $60 \%$ intrinsic parameter contribution solution, and a 200 -fold error change in the $220 \%$ intrinsic parameter contribution solution (Figure 29A, right blue boxplots).

Extrinsic flexor related stability was observed most in the $1 \%$ intrinsic contribution solutions for healthy locomotor output, while the same solution set tended to be least stable along the extrinsic extensor related parameters (Figure 29B, top panel). The $1 \%$ intrinsic solutions of hemiparetic output were the most unstable (Figure 29B, middle panel), and a readaptation optimization produced solutions which were comparatively more stable along the flexor integrator related parameters, than the extensor integrator related parameters (Figure 29B, bottom panel). The 
results of perturbing different flexor and extensor integrator related parametric pairs is summarized in Figure S3.

The perturbation analysis was extended to separate parametric pairs contributing to a single halfcenter, and the output of the differential equation was analyzed by a modified error function which included only the root-mean-square difference from the optimized solution and the perturbed solution. Upon perturbing the parameters by linear increments from $-10 \%$ to $+10 \%$ (or $-20 \%$ to $+20 \%$ ) of the parametric weight for optimum solutions, the resulting root-mean-square differences were color plotted on a two-dimensional axis, where the perturbation of an extrinsic parameter contributing to a single flexor integrator was plotted in one dimension ( $y$ axis), and a second parameter contributing to the extensor integrator was plotted in the second dimension ( $\mathrm{x}$ axis). The results revealed that for the $1 \%$ intrinsic contribution solution, there is a tendency for the RMS function to vary linearly with linear parametric change, whereby an optimization minimum could be achieved if the first parameter was perturbed by ' $n$ ', while the second parameter was perturbed by '- $n$ ' (Figure 30A). This was found to be true only for the solution set of $1 \%$ intrinsic parameter contribution, while increasing the parametric contribution of the intrinsic parameters to $30 \%$ revealed that the solution space becomes nonlinear (Figure 30B). Ultimately, perturbing the intrinsic extensor-extensor parameter ' $r 42$ ' in the $1 \%$ intrinsic parameter contribution set had no effect on the root-mean-square deviation from the optimized solution, indicating that the contribution of the intrinsic parameters to the $1 \%$ solution may largely be neglected. This was not the case, however, for the $30 \%$ intrinsic parameter contribution solution sets, which despite the nonlinearity of the solution space, produced significant alterations to the root-mean-square output from the optimized state (Figure $\mathbf{3 0 C}$, top panel). When comparing the function of the selfsustainment parameters on integration, it is interesting to note that while the $1 \%$ intrinsic solution becomes perturbed in a linear fashion similar to the perturbations of the extrinsic parameters, perturbations to the $30 \%$ intrinsic solution demonstrate that even more optimal states can be 
achieved away from the predicted optimum, indicating that the linear optimization Nelder-Mead algorithm may be insufficient at producing an optimized solution along the self-sustainment parameters.

A.
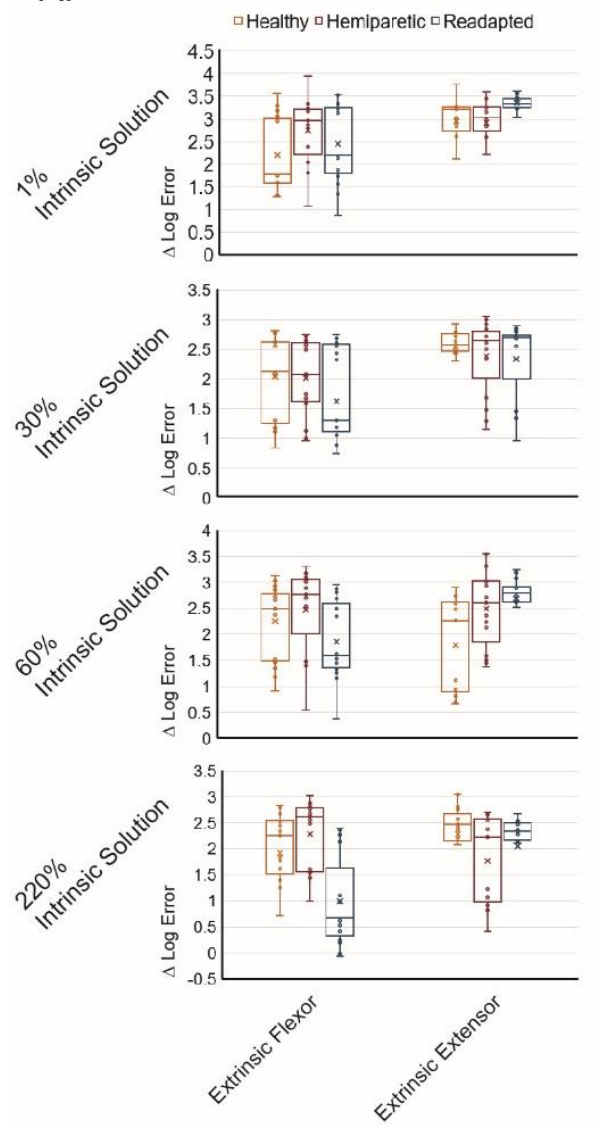

B.
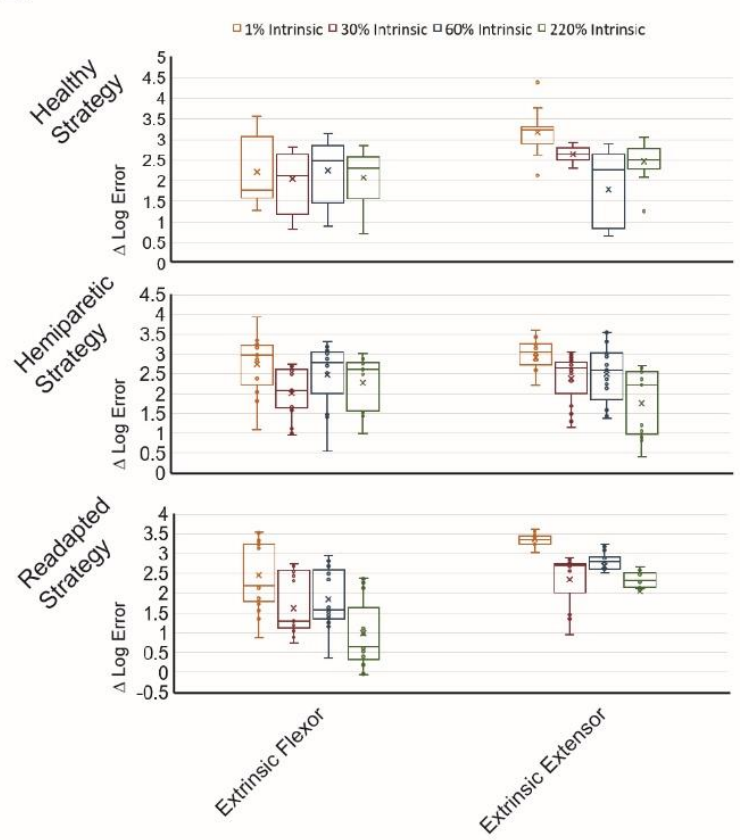

Figure 29. Error change is distributed among flexor and extensor integrator related parametric subsets. 10 SNR noise was introduced to different flexor and extensor integrator related parameters, and the resulting solution set was analyzed for error differences from the optimized solution, and the error change was plotted on a logarithmic scale. A. Comparative error changes from noise introduction to different parametric subsets among healthy, hemiparetic, and readapted solutions. Top panel $-1 \%$ relative intrinsic parameter contribution; second panel $-30 \%$ relative intrinsic parameter contribution; third panel $60 \%$ relative intrinsic parameter contribution; bottom panel $-220 \%$ relative intrinsic parameter contribution. B. Comparative error changes from noise introduction to different parametric subsets among solutions of different relative intrinsic parameter contribution. Top panel - solutions of healthy output; second panel solutions of hemiparetic output; bottom panel - solutions of readapted output. 
A.

B.
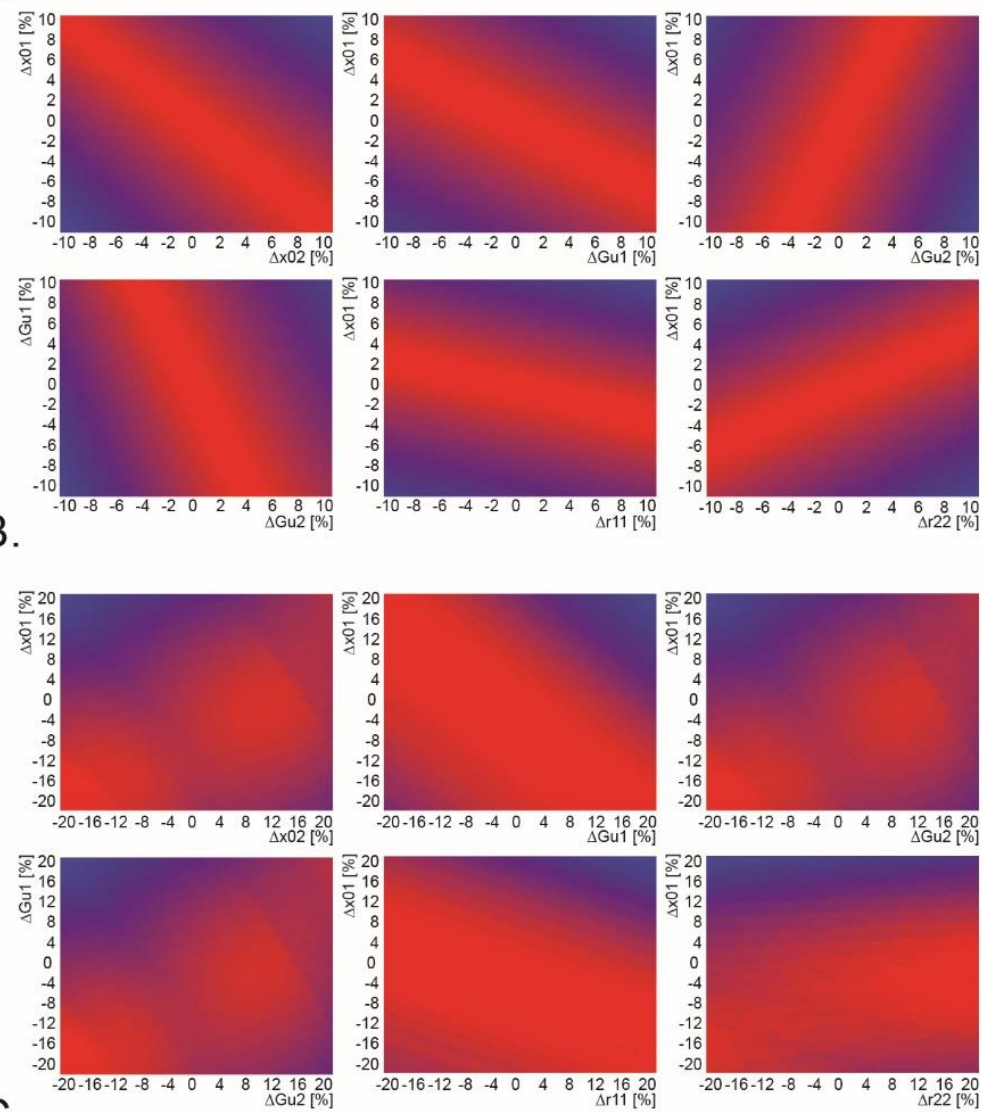

C.

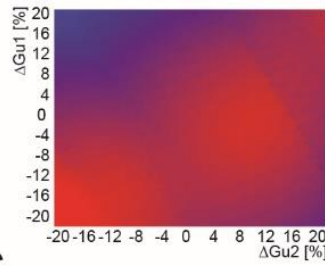

$1 \%$ Intrinsic Solution

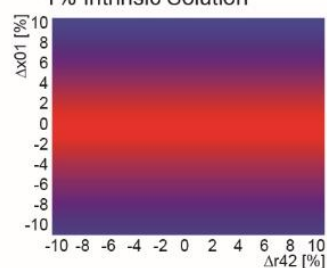

$30 \%$ Intrinsic Solution
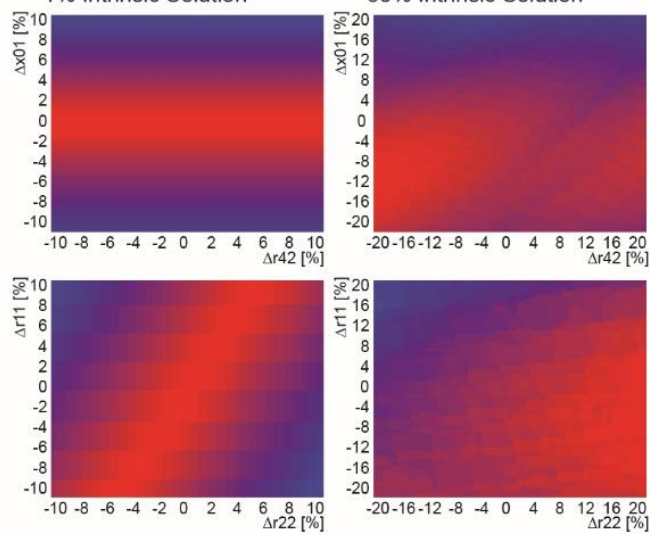

Figure 30. Solution space relationships between different pairs of extrinsic and intrinsic parameters for single half-center output. A. Different pairs of extrinsic, selfsustainment, or intrinsic parameters were subjected to $10 \%$ parametric variation imposed on an optimized 1\% intrinsic parameter solution. B. Different pairs of extrinsic, selfsustainment, or intrinsic parameters were subjected to $20 \%$ parametric variation imposed on an optimized $30 \%$ intrinsic parameter solution. C. Extrinsic parameter ' $x 01$ ' variation paired with variations of intrinsic right-to-left extensor-toextensor integrator parameter ' $r 42$ ' (top row), and variations of left half-center related ' $r 11$ ' and 'r22' parameters (bottom row). \%RMS - percent root-mean square difference between optimized and sub-optimal solutions from the maximum RMS deviation for that range.

\subsection{Transient ischemic stroke preferentially affects hemiparetic dorsiflexor muscle}

A 30 to 45 minute unilateral transient brain injury through the $\mathrm{MCAO}$ procedure was sufficient to induce unilateral damage to the motor cortex and basal ganglia in wild-type C56BL-7 mice, but insufficient to cause damage to the autonomic regulatory centers of the thalamus and 
hypothalamus (Figure 31A). This indicates that while centers which regulate feeding and temperature homeostasis are relatively uninjured, the perturbation is sufficient in inducing damage to the motor cortex and basal ganglia, which limits the impediments to locomotion due to damage to other brain centers of general homeostatic changes. 24 hours after injury, unilateral ischemic insult to the brain induces accumulation of total FoxO3a protein in hemiparetic muscle only. This cell-stress response protein is predominately found in a dephosphorylated and hypoubiquitinated state, as evidenced by a faster migratory pattern (Figure 31B). While the paretic tibialis anteriores are subjected to this insult, nonparetic and hemiparetic gastrocnemi are spared from injury, indicating that while the ischemic brain injury upregulates the cell-stress FoxO3a pathway on the paretic dorsiflexors, the paretic plantar flexors seem to be spared from this stress. 24 hours after transient ischemic brain injury, there is also a marked decrease in p53 protein levels in hemiparetic tibialis anteriores dorsiflexors (Figure 31D). This component of the DNA damage response pathway is associated with elevated muscle-specific RING-finger 1 (MuRF-1) E3 ubiquitin ligase as well, which is observed in both the dorsiflexor and plantar flexor populations (Figure 31C). 48 hours after transient ischemic brain injury, the hemiparetic dorsiflexor population starts to exhibit Sirt1 protein loss (Figure 31D), which is not reserved for the full-length protein alone, but also affects the more slowly-migrating ubiquitinated species (Figure 31E). The loss of this deacetylase is associated with increased levels of acetylated PGC1a, which migrated between the 80 and 110 kDa markers (Figure 31F). Furthermore, the acetylation status of the $\mathrm{p} 65$ protein fragment of the NF-к $\beta$ proinflammatory signaling pathway was found to be elevated (Figure 31G), which was concomitant with the increased levels of total p65 protein (Figure 31C). Western Blotting of muscle lysates demonstrated a particular trend of upregulation of muscle wasting markers in the paretic limbs after unilateral ischemic brain injury. Primarily, though MuRF-1 is a type II muscle associated ubiquitin ligase, increased MuRF-1 protein content was predominately observed in the tibialis anterior muscles, whereas the increase was observed only moderately in the plantar flexor muscle group, such as the gastrocnemi, 
plantares and solei muscles. This effect, however, was observed only moderately in the nonparetic limb muscles (data not shown).

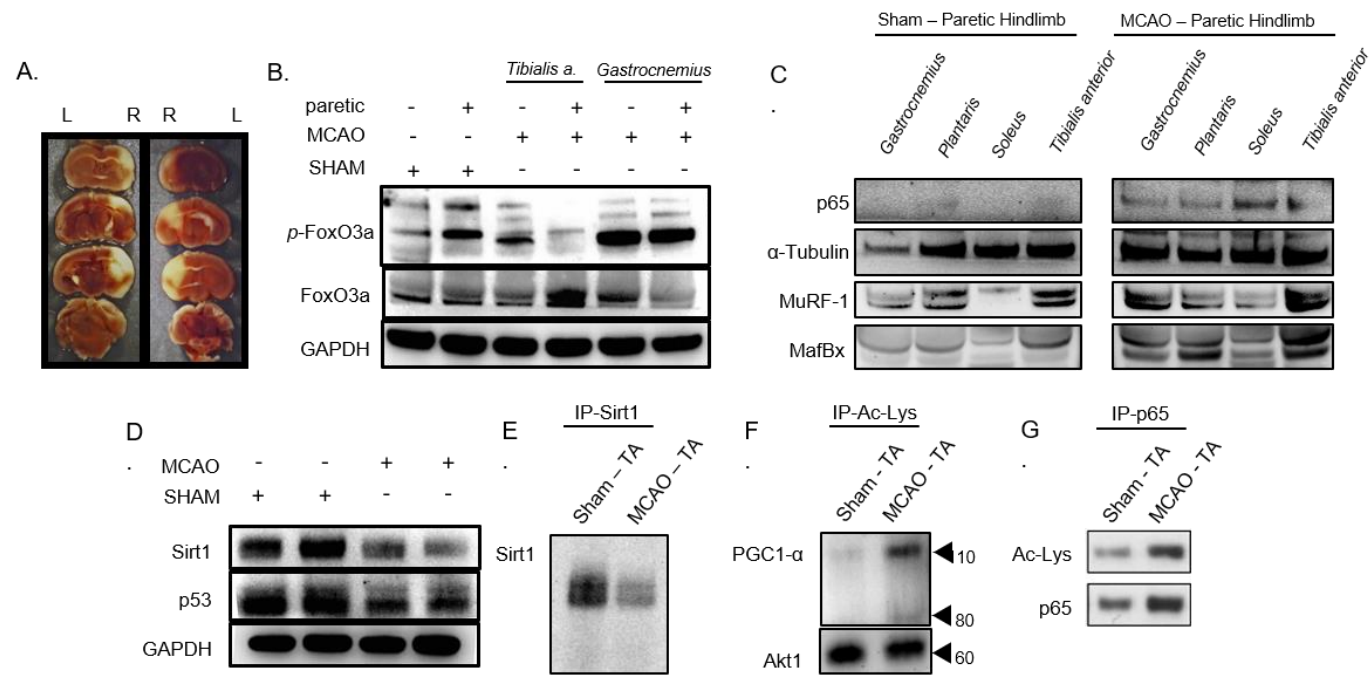

Figure 31. The cell stress pathway demonstrates preferential dorsiflexor impairment in hemiplegic muscle after ischemic stroke. A. A 30-45 minute transient middle cerebral artery occlusion induces damage to the cortex and basal ganglia, while sparing central thalamic and hypothalamic centers. B. 24 hours after unilateral transient ischemic brain injury, the FoxO3a apoptotic signaling pathway becomes upregulated, resulting in decreased phosphorylation levels of FoxO3a protein, and an upregulation of total FoxO3a protein in paretic dorsiflexor muscles. The FoxO3a pathway does not become upregulated on the plantar flexor muscle group. C. MafBx/Atrogin-1 and MuRF-1 E3 ubiquitin ligase protein levels are increased in hemiparetic dorsiflexors and plantar flexors, and an increase in the levels of p65 NF-k $\beta$ fragment is identified in plantar flexors and dorsiflexors. D. Levels of Sirt1 and p53 in hemiparetic Tibialis anterior muscles. E. Immunoprecipitation of Sirt1 demonstrates the loss of multiple Sirt1 protein species 48 hours after transient middle cerebral artery occlusion (MCAO). F. Sirt1 loss is associated with accumulation of hyperacetylated PGC1 $\alpha$, which is inhibitory for transcription factor function. Levels of GAPDH, or $\alpha-$ tubulin were used as a loading control. G. The levels of NF-к $\beta$ p65 fragment are elevated and hyperacetylated in hemiparetic muscle 48 hours after ischemic brain injury. TA - tibialis anterior muscles. All samples were obtained from procedures on wild-type animals. Sham - samples from sham operated animals; MCAO - samples from 30 minute middle cerebral artery occlusion animals. 


\section{DISCUSSION AND RECOMMENDATIONS}

\subsection{Dorsiflexor impairment is associated with injury to the descending drives of the central pattern generator}

In contemporary neuroscience, there have been several integrative theories in regards to the purpose of having the central nervous system arranged in such a fashion that it may be possible to integrate reactive as well as predictive modes of locomotion. Herein, it would stand to reason that lower, reactive, forms of optimization of a locomotor pattern may need to be eliminated in order to investigate the predictive factors influencing locomotion. For that purpose it is important to eliminate environmental perturbations which may cause reactive readjustment of the locomotor output. In these experiments, it was therefore important to implement rigorous training routines for the experimental subjects, and as such, the subjects needed to present mastery of the locomotor tasks. For that reason, only locomotion which was identified to be of sufficient 'proficiency' was used for further analysis (continuous walking, arched back and raised tail).

Certain factors of the motor actuators contribute to locomotor instability characteristic of hemiparetic gait. Recently, it has been demonstrated that both the MuRF-1 and MafBx E2 ubiquitin ligases exhibit increased levels of mRNA expression in paretic muscle in rodents which have undergone a transient middle cerebral artery occlusion, which points to the ubiquitous role of MuRF-1 in multiple sarcopenic phenotypes, as well as the pathophysiological phenotype associated with hemiparetic muscle ${ }^{18}$. Both MafBx and MuRF-1 share a common promoter FoxO element, which becomes active in various other forms of muscle wasting. In the data from this study, the sarcopenic phenotype is associated predominately with the dorsiflexor population, and is characterized by the pathophysiology of denervated muscle.

This observable muscle pathology is not necessarily a limiting factor of the study. Namely, the motor unit and the underlying motor neuronal as well as pre-motor neuronal connectivity within the central nervous system do not exist in isolation. Rather, they are a apart of a dynamic system 
of homeostatic regulation with marked retrograded influence of muscle fiber composition to the function of the $\alpha$-motor neurons ${ }^{242}$. As such, and when considering the dysregulation of the motor system as a whole, it is important to consider the nature of the motor actuators, and to characterize any damage associated with hemiplegic muscle pathophysiology in relation to the damage of the central drives. For that purpose, considering the fact that alterations to intrinsic centers of pattern generation have been known to induce a sarcopenic phenotype bilaterally, especially following spinal cord injury ${ }^{243}$, it remains important to address the unilaterality of muscle pathophysiology after ischemic cerebral vascular injury. Rather, the hemiparetic muscle deficits in relation to the contralaterality of ischemic brain injury, and in the absence of marked ipsilateral muscle injury, is indicative of direct correlations of the descending corticospinal input to the target rhythm generating center. Such corticospinal injury has been known to induce a reduction in motor unit activity ${ }^{25,244}$, and even $\alpha$-motor neuron death ${ }^{23}$, the latter of which has been scarcely addressed in literature. In that sense, perhaps paying attention to morphological differences of hemiparetic atrophy responses may be point to the neural correlates of impediment in a highly complex architecture such as the central pattern output centers of the spinal cord.

\subsection{Numerical solutions demonstrate multiple strategies for solving locomotor output}

From these simple manipulations of the architecture of connectivity, it would appear inherent that solutions to the differential equation can be obtained numerically even from sets which employ more parameters. These solutions are further dependent on the initial conditions, as well as the range of locomotor speeds the model needs to emulate. It should be expected that such models will have a great number of solutions, but some general properties of the solutions may be expected. In essence, a locomotor central pattern generator could be regulated through extrinsic means, whereby the bilateral coupling inputs between the half-centers could be negligible. Vice versa, optimal locomotor behavior could be obtained through manipulations of only the intrinsic 
parameters, which could potentially account for the great degree of biological connectivity within the spinal cord.

To avoid great solution space variation, the initial conditions were kept equivalent for all optimization runs. In addition to a solution set obtained from symmetrical initial conditions, which employed equivalent bias for all intrinsic and extrinsic parameters, a second set of initial conditions was employed, which used a bias on the integrators simulating flexor activity. This set did not produce any difference in the trend of parametric alteration, and the parametric difference between healthy and paretic locomotion was predicted to have remained flexor-dependent. The effective size variation was not predicted to be distributed among sets of intrinsic or extrinsic parameters, however, which points to different modes of reaching the optimal state space, other than linear input from the motor cortex. This does not seem to be the case if we take into consideration the parametric weight change of the different solution sets which produce paretic locomotion.

Interestingly, each different type of solution, and depending on the relative contribution of the intrinsic parametric subset, is prone to different sensitivity to parametric change. On the one hand, the solution space with relatively little and largely insignificant contributions of the intrinsic parameters is capable of predicting that the hemiparetic half-center is subjected to altered input from extrinsic parameters is prone to greater instability when perturbing parameters contributing to the flexor output simulating integrator.

\subsection{Numerical solutions predict flexor integrator activity as the culprit of foot-drag in paretic locomotion}

An interesting observation from the modeled behavior of asymmetric locomotion induced by ischemic brain injury, is the fact that despite no effective size redistribution of either intrinsic or self-sustained parameters obtained from numerical solutions, the predicted weight change of the 
extrinsic parameters was indicative of the lessened contribution of the drives to the centers simulating flexor activity, which ultimately produces paretic locomotor characteristics. This result is only true if the relative contribution of intrinsic parameters is assumed to be approximately $1 \%$ of the size of the extrinsic parameters. This observation is also biologically significant, as foot (paw) drag is one of the key characteristics of paretic locomotor behavior associated with dorsiflexor impairment in ischemic injury of the brain ${ }^{22}$, as well as spinal cord injury ${ }^{217,245}$, and often represents the target behavior of rehabilitation strategies ${ }^{216}$.

Such predictions can serve for further investigations of the true biological nature of the central pattern generator, as well as the contributions such networks have on the neuromuscular system overall for the purpose of assessment of impairment, as well as future contributions mathematical modeling may have on rehabilitation strategies. If the observations of the lateralization of muscle atrophy following ischemic brain injury are taken into consideration, it would appear that despite any reorganization of the intrinsic connectivity of the lower locomotor centers, integrator activity predominately relies on the weight of the extrinsic drives which contribute to dorsiflexor function. Interestingly, these drives are not identified in solutions of healthy lateralized gait, indicating that healthy gait utilizes multiple strategies of optimization of locomotion. Conversely, paretic gait is the result of impairment of the extrinsic drives governing flexor function, as well as contralateral flexor-extensor coupling.

Lastly, further reduction of connectivity that relies on solely extrinsic or intrinsic parametric contribution reveals that in paretic locomotion (1) multiple different strategies have to be employed in order to stabilize locomotion driven solely by intrinsic lower center connectivity, and (2) strategies that neglect intrinsic connectivity have to be driven by ipsilateral stabilization within the half-centers. This is not the case for healthy locomotion, however, as there appears to be no particular trend in modifying healthy gait in order to produce different locomotor patterns. 
From this, it would follow that healthy gait may rely on the principle of least resistance, that is to say, that healthy neurological activity would require the lowest possible parametric perturbations in order to meet environmental demands on locomotion. In paretic locomotion, these demands become significantly greater, posing a greater challenge to the paretic corporeal side (contralateral to the injured cortex). This is consistent with findings that dorsiflexor activity is more significantly impaired during the foot-drag behavior, as well as the decreased number of active motor units ${ }^{246}$ of the dorsiflexor population ${ }^{247}$. There is also some evidence demonstrating that there is not only a functional, but also a physical loss of lower motor neurons ( $\mathrm{aMNs})^{23}$ which may further result in classical muscle atrophy ${ }^{18}$, and this condition exhibits a pathophysiological bias to the paretic side. The find that dorsiflexors are preferentially involved in stroke-related muscle atrophy hints that the involvement of the central nervous system contributions to the dorsiflexor group may be more significantly involved in regulating locomotion. Therefore, any holistic models of locomotion need to rely on other observations from biology in order to ascertain the proper mode of driving such an inherently complex system.

\subsubsection{The hemiparetic strategy lies close to an alternative solution space of healthy locomotion}

In order to achieve the strategy which lies closest to the hemiparetic solution space, the existing 'hemiparetic' strategy needs to be readapted in several ways. First, the difference in driving a 'hemiparetic' CPG from that of the new adaptation relies on almost all parametric subsets, save for the self-sustained intra-integrator coupling, and the left-diagonal flexor-to-extensor coupling (Figure 24D and Figure 25D). Considering that these drives are impaired most in solution sets of asymmetric locomotion, it appears pertinent to note that avoiding modulating these drives when attempting to produce a symmetric solution to the CPG may be of key interest when trying to readapt the 'hemiparetic' central pattern generator. As the descending, extrinsic, drives governing flexor function appear to be dysregulated, the results from this study indicate that the mode of 
driving a novel readapted strategy must rely on modulating not just one, but almost all sets of parameters. Therefore, any rehabilitative measure needs to focus on all parametric sets, except for the ones that are found to be impaired due to damage to centers governing the descending input, as well as inter-center connectivity. The solutions obtained from initial conditions that otherwise reproduce asymmetric locomotion akin to the one observed in hemiparetic locomotion reveal several general observations. First, it is possible to obtain oscillatory patterns resembling healthy locomotor output from a solution set that is theoretically closest to the 'hemiparetic' solution. Yet, this novel strategy relies on readapting multiple sets of parameters to the point that the intrinsic set of parameters may be completely disregarded. This conclusion, however, is only true when trying to compare 'paretic-to-healthy' solutions to solutions of healthy locomotion originating from symmetric initial conditions generating equivalent swing and stance phase characteristics.

One strategy may focus on the function of integrator self-sustainment (Figure 24A and 25A). For the purpose of clinical rehabilitation, this task may have to focus on retraining the hemiparetic flexor to hyper-reflexive self-sustainment, meaning that even small input weights from the descending drives would have sustain faster flexor-phase execution (integration) that would put small regard to intra-center connectivity (intrinsic connectivity), and little regard to modulating the self-sustainment of the extensor phase. Alternatively, and in order to avoid placing greater burden on the already impaired left-to-right flexor-extensor coupling, the self-sustainment parameters, as well as the overall direct inputs to the flexor integrators, greater focus needs to be placed on readapting extrinsic and intrinsic inputs that would modulate the function of the left half-center indirectly through modulating right-to-left flexor-extensor couplings (Figure 24C and 25C).

When analyzing the solution space for healthy and hemiparetic locomotion, it becomes evident that the holistic model predicts preferential impairments to flexor and flexor-related couplings (Figure 22E). From this observation, a reasonable conclusion suggests that in order to readapt 
the locomotor control system to produce healthy output, it would be necessary to utilize strategies which avoid the use of the impaired half-center couplings. For that purpose, analyses of task space readaptation through the use of conventional error reduction methods reveals that parametric weight distribution strategies which differ from the hemiplegic strategies through other means than the injured couplings may prove to be the most biologically significant models.

One such model solution set is represented by the flexor-biased initial condition derived solutions of healthy and hemiparetic locomotion, termed $\mathrm{IC}^{\mathrm{F}}$ and $\mathrm{IC}^{\mathrm{FH}}$. Parametric alterations derived from $I^{S}$ and $I C^{F}$ are indifferent in their predicted mode of parametric weight redistribution in order to achieve hemiplegic asymmetry, and suggest that the hemiparetic flexor-related drives are the main culprit of stroke-related asymmetry (Figure 22E); however, the readaptation space for these different solutions is substantially different (Figure 25C). Readaptation strategies for hemiplegic solutions obtained from symmetric $I C^{S}$ suggests the obvious - namely, that if one were to just readapt the hemiparetic flexor drives, there would be no problem, and the locomotor pattern would return to normal. This may not be possible if the descending drive centers originating in the motor cortex and basal ganglia are permanently injured, as would be the case for ischemic or traumatic brain injury. A more promising solution for locomotor pattern readaptation is suggested by the solutions obtained from $\mathrm{IC}^{\mathrm{F}}$ and $\mathrm{IC}^{\mathrm{FH}}$. These solutions suggest that the most optimal means of retraining the hemiplegic locomotor CPG is to avoid retraining the hemiplegic flexor-related drives completely. Furthermore, these results would suggest that the retrained task-space naturally lies in closest proximity to the hemiparetic output producing task-space. Together, these results suggest that an inherent difference in driving the central pattern generator for locomotion may also contribute to the mode of impairment, which would be another factor to consider for the purpose of studying the varying degrees of success in the clinical rehabilitation setting. 


\subsection{A precise foot-placement task requires proper modulation of the descending drives}

Though other numerical and analytical solutions of reduced versions of the holistic model of central pattern generation demonstrated herein have demonstrated that an optimum solution of an 11 parameter model may have equivalent percentile extrinsic-to-intrinsic drive contribution for behaviors of different limb cycle velocities ${ }^{248}$, this may not be true for a precise foot-placement task which does not involve different cycle speeds on each limb. In the present modeling study, it was demonstrated that a solution which converges on approximately $1 \%$ contribution of the intrinsic connectivity of the left and right rhythm generating half-centers is most capable of predicting parametric alterations which render unto a healthy locomotor output solution the characteristics of hemiparetic locomotor rhythms. This would imply that while model solutions which utilize greater reliance on the intrinsic connectivity parameters of 30\% (or more) may simply be able to reproduce asymmetric rhythms akin to hemiparetic locomotion by insignificant modifications of many subsets of parameters, ultimately producing no statistical significance for the change of any parametric subset.

This find is in line with the present literature on the topic. Namely, over the course of the last 30 years, the work of Irina Beloozerova and her lab has demonstrated that higher centers of motor control, such as the motor cortex and basal ganglia, are most implicated in task that require precise limb manipulations during locomotion ${ }^{235,236,249,250}$. Furthermore, studies by Trevor Drew et al. have also demonstrated that the dorsiflexor related function during the production of obstacle avoidance trajectories is most significantly impaired following the GABA-receptor agonist muscimol injections to the motor cortex in the cat $^{221}$, which is a comparable manipulation to that of transient ischemic ablation of the motor cortex. Finally, some interesting studies on pattern alteration come from studies on the ablation of crossed commissural connections in the turtle spinal cord for the purpose of evaluating the rhythmicity of swimming ${ }^{251}$. These studies 
demonstrate that even a longitudinal resection of the commissural pathways in the lumbar segments of the spinal cord of turtles does not contribute significantly to the rhythmicity of locomotor output during swimming. Whether the reptilian model of spinal pattern generation is comparable to that of mammals remains to be studied, and unfortunately, this experiment has not been performed in mammals to date. Most spinal cord injury or resection studies focus on the ablation of corticospinal and reticulospinal pathways, and a longitudinal resection study on the spinal cord for the purpose of evaluating its contribution to rhythmicity in vivo remains to be addressed. In vivo and in situ studies of spinal cord explants, however, reveals that the spinal cord populations possess an inherent property of pattern generation, but it is also true that this rhythm generation can be modified by different means, including the alteration of individual commissural and intersegmental pathways through the contribution of modulations originating from higher centers of motor control.

\subsubsection{Intrinsic parameters stabilize the locomotor output}

The data from the stability analysis under various signal-to-noise ratios reveal several things. First, it is possible to obtain solutions from multiple task-space positions of increased intrinsic parameter contribution relative to that of the intrinsic parameters. These solutions, however, do not respond perfectly linearly to increased tonic input from ' $u$ ' (comparatively to the $1 \%$ intrinsic solutions, Figure 28, B through E), and they do not achieve a linear minimum. Rather, the vicinity of the optimized task space around a single solution tends to produce non-linear relationships to linear parametric perturbations (Figure 30). Moreover, linear perturbations to parametric pairs tends to produce little deviation from the optimum, indicating that while the solution space around individual parametric sets is non-linear, increasing the relative contribution of the intrinsic parameters produces a desired property for the solution space. Namely, these saddle points around the optimized solution space may be the source of increased stability associated with greater intrinsic parameter contribution (Figure 29A). In concert, these results could account for 
the clinical observation of rehabilitative strategies aimed at restoring locomotor function through the reengagement of spinal centers responsible for locomotion, which while capable of restoring gross locomotor function, they are largely insensitive to fine-tuning and precise locomotor tasks. 


\section{Supplementary Figures}

A.
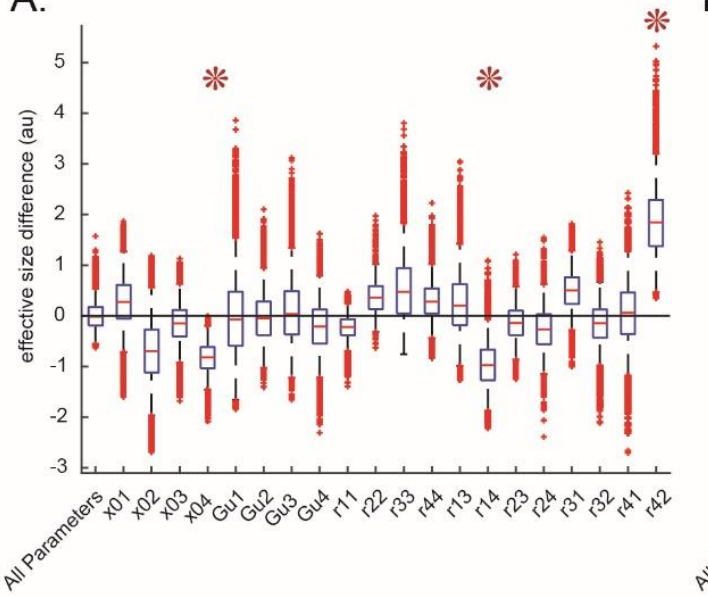

D.

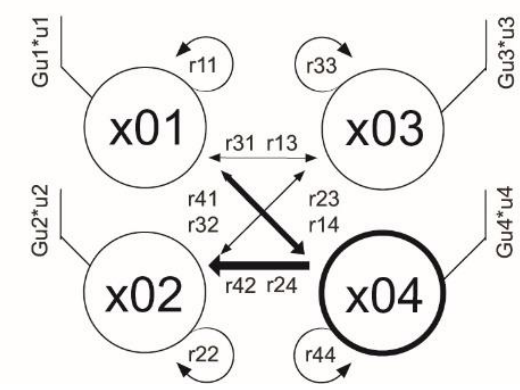

B.

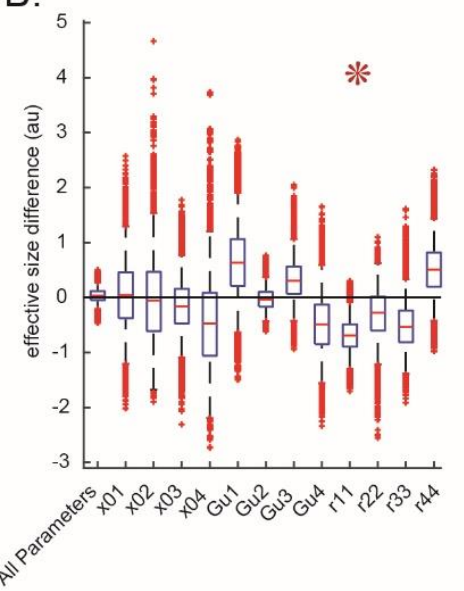

C.

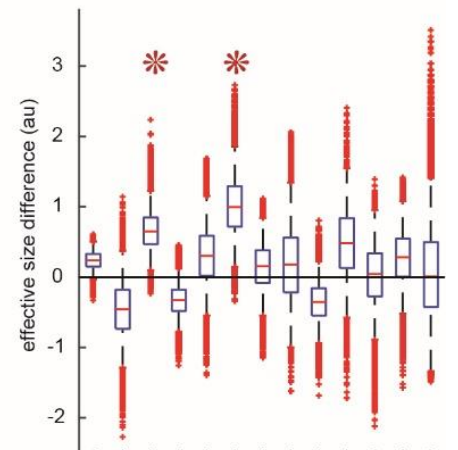

F.
E.

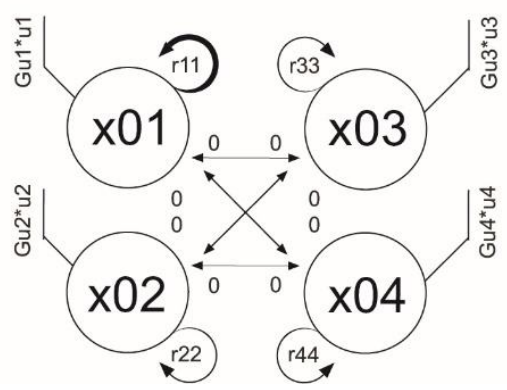

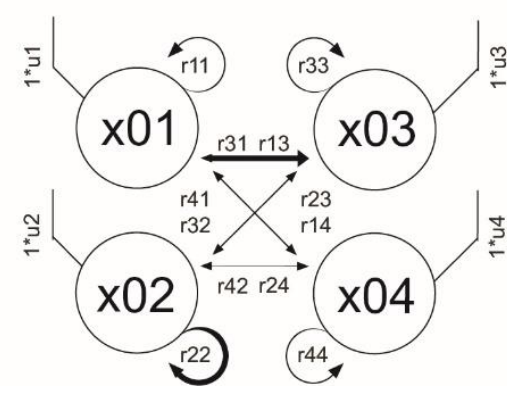

Figure S1. Single parameter analysis for parametric alteration between healthy leftpreferred and right-preferred gait. A. Single parameter analysis for the expanded 20 parameter model shows that altering the weight of three parameters ' $x 04$ ', ' $r 14$ ', and ' $r 42$ ', can modulate the model output from a left-preferred gait pattern to a right-preferred gait pattern. B. Single parameter analysis for the Sherrington type model reveals that the left-preferred gait pattern can be modified into a right-preferred gait pattern through the ' $r 11$ ' parameter alone. $\mathbf{C}$. Single parameter analysis for the Brown type model reveals that the left-preferred gait pattern can be modified into a rightpreferred gait pattern through modulating the ' $r 22$ ' and ' $r 13$ ' parameters alone. D. through F. graphical summary of the analyses from $\mathbf{A}$. through $\mathbf{C}$. 

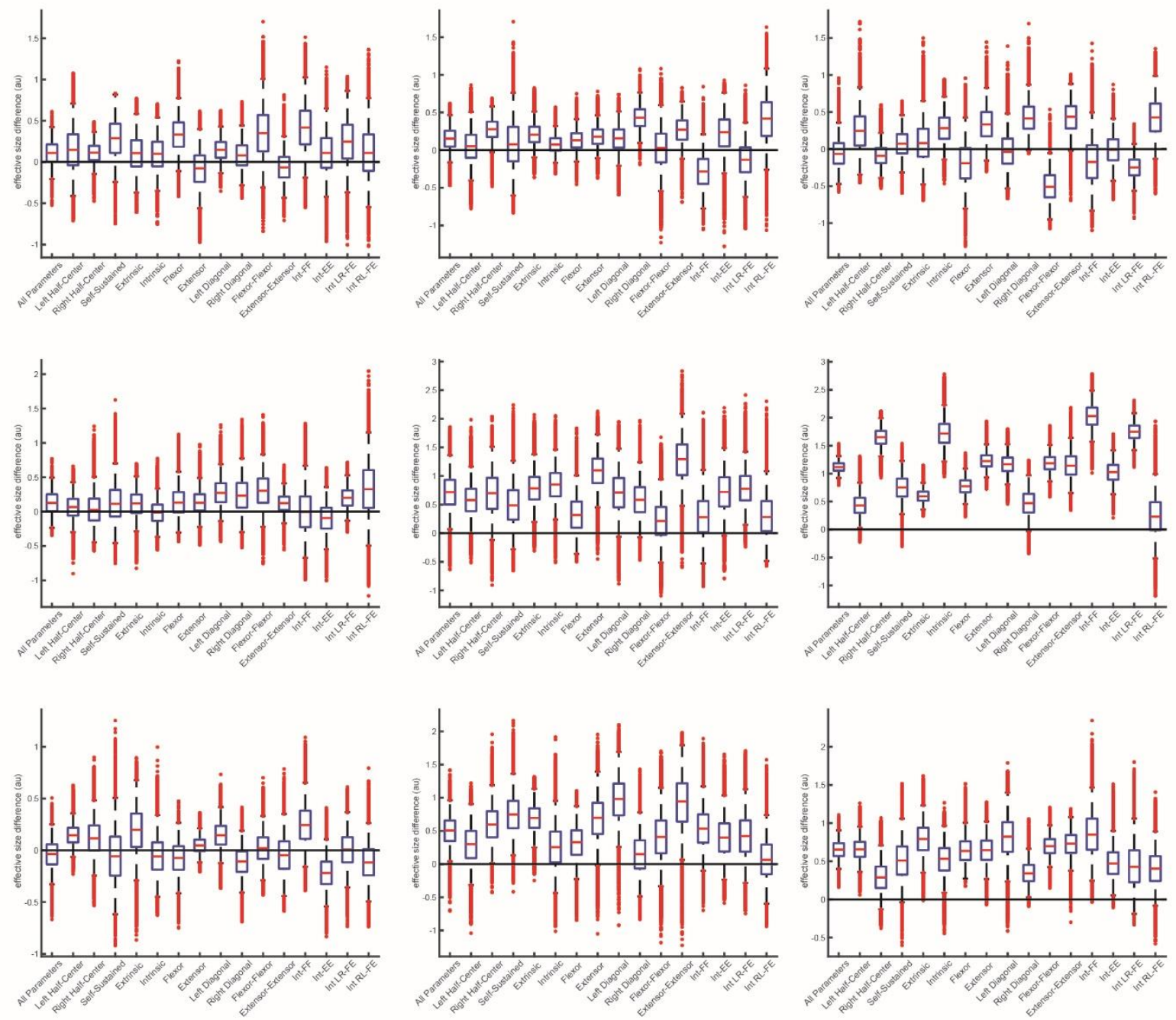

Figure S2. Parametric alteration trends from single parameter analysis of solutions of varying intrinsic parameter contributions. Effective size distribution was analyzed for significance by the Bootstrap method from healthy to hemiparetic solutions (left column), hemiparetic to readapted solutions (middle column), and healthy to readapted solutions (left column). Analysis on $30 \%$ intrinsic contribution solutions (top row), $60 \%$ intrinsic contribution solutions (middle row) and $220 \%$ intrinsic contribution solutions (bottom row) were used to generate the graphic in Figure 26B. Effective size differences were calculated by Equation 12 between each designated solution set group indicated in individual panels. All effective size differences were evaluated for statistical significance by the Bootstrap method at a boot number of 100,000 . 
A.
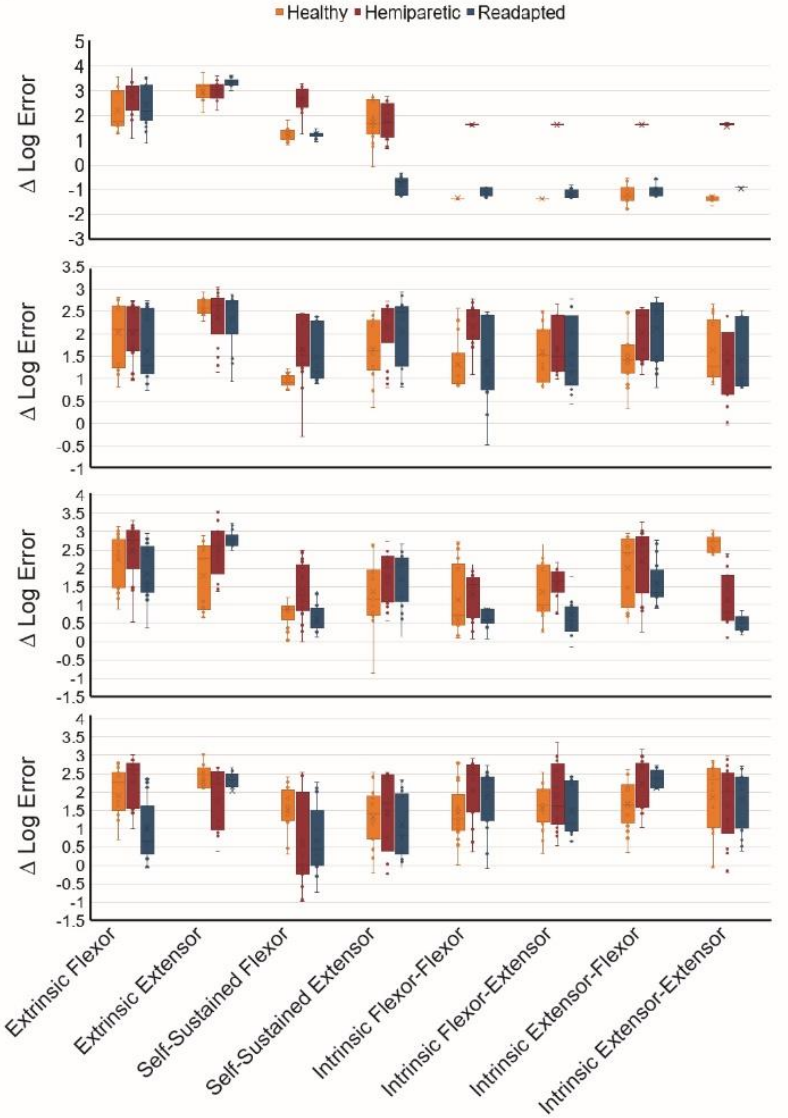

B.
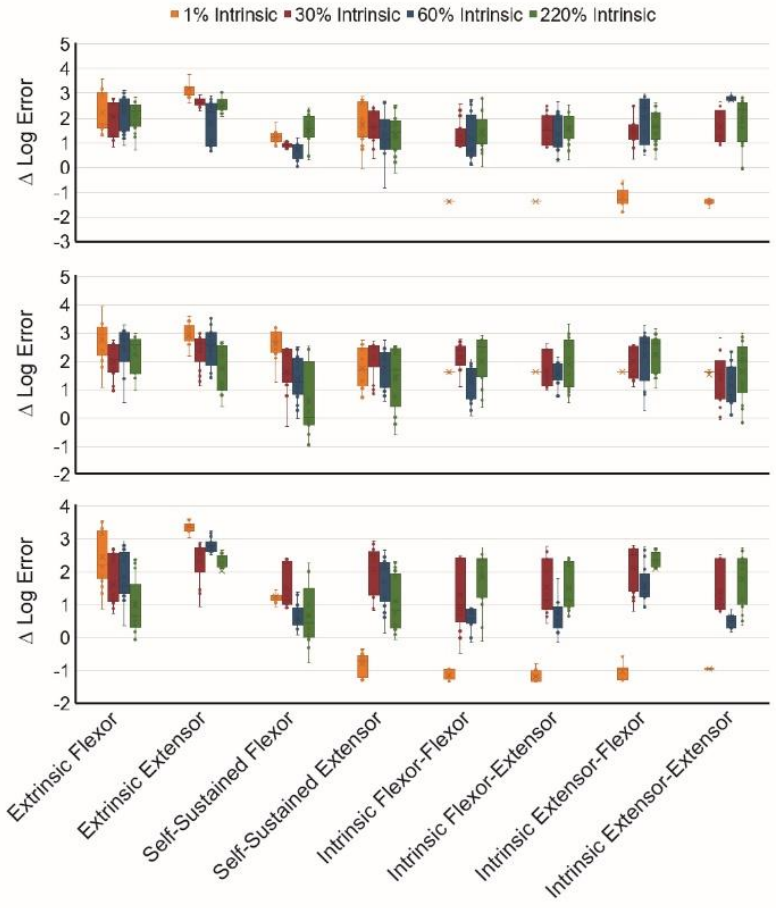

Figure S3. Logarithmic error differences between optimized and suboptimal solutions under 10 SNR, and in relation to Figure 29. 10 SNR noise was introduced to different flexor and extensor integrator related parameters, and the resulting solution set was analyzed for error differences from the optimized solution, and the error change was plotted on a logarithmic scale. A. Comparative error changes from noise introduction to different parametric subsets among healthy, hemiparetic, and readapted solutions. Top panel $-1 \%$ relative intrinsic parameter contribution; second panel $-30 \%$ relative intrinsic parameter contribution; third panel $-60 \%$ relative intrinsic parameter contribution; bottom panel $-220 \%$ relative intrinsic parameter contribution. B. Comparative error changes from noise introduction to different parametric subsets among solutions of different relative intrinsic parameter contribution. Top panel solutions of healthy output; second panel - solutions of hemiparetic output; bottom panel - solutions of readapted output. 
A.

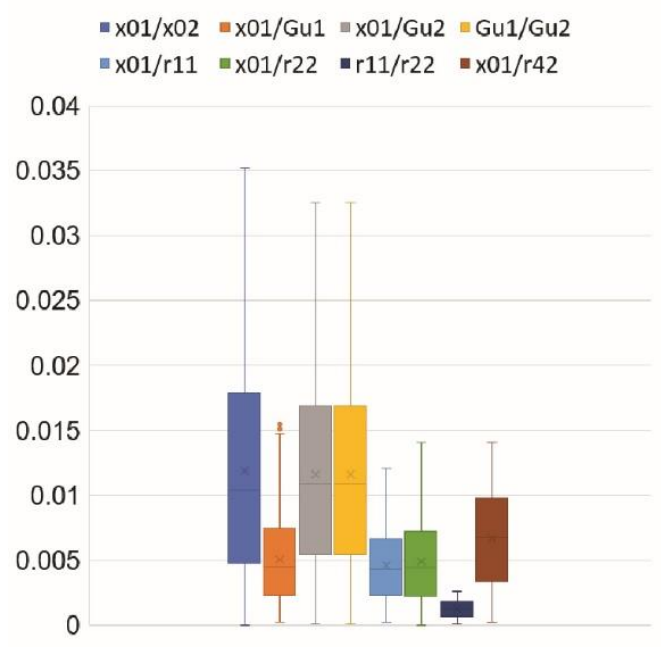

B.

$\llbracket \times 01 \times 02 \approx x 01 / G u 1=x 01 / G u 2 \square G u 1 / G u 2$

$=x 01 / r 11=x 01 / r 22=r 11 / r 22=x 01 / r 42$

0.06

0.05

0.04

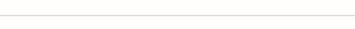

0.03

0.02

0.01

0

Figure S4. Root-mean-square differences between optimized solutions and suboptimal solutions from Figure 30. A. $10 \%$ linear perturbations to $1 \%$ relative intrinsic parameter contribution solution sets. B. $20 \%$ linear perturbations to $30 \%$ relative intrinsic parameter contribution solution sets. 


\section{References}

1. Squire, L. R. et al. Fundamental Neuroscience. (Elsevier Academic Press, 2008).

2. Scherbakov, N. \& Doehner, W. Sarcopenia in stroke - facts and numbers on muscle loss accounting for disability after stroke. J. Cachexia. Sarcopenia Muscle 2, 5-8 (2011).

3. The European Registers of Stroke Investigators, E. Incidence of Stroke in Europe at the Beginning of the 21st Century. Stroke 40, 1557-1563 (2009).

4. $\quad$ Kalogeris, T., Baines, C. P., Krenz, M. \& Korthuis, R. J. Cell Biology of Ischemia/Reperfusion Injury. Int Rev Cell Mol Biol 298, (2012).

5. Wang-Fischer, Y. \& Koetzner, L. Animal models of ischemic stroke - a historical survey. Manual of Stroke Models in Rats (CRC Press, 2009).

6. McCullough, L. D. \& Liu, F. Middle cerebral artery occlusion model in rodents: Methods and potential pitfalls. J. Biomed. Biotechnol. 2011, (2011).

7. $\mathrm{Hu}, \mathrm{H}$. et al. Mitochondrial impairment in cerebrovascular endothelial cells is involved in the correlation between body temperature and stroke severity. Aging Dis. 7, 14-27 (2016).

8. Nour, M., Scalzo, F. \& Liebeskind, D. S. Ischemia-reperfusion injury in stroke. Interv. Neurol. 1, 185-199 (2012).

9. Kalogeris, T., Bao, Y. \& Korthuis, R. J. Mitochondrial reactive oxygen species: A double edged sword in ischemia/reperfusion vs preconditioning. Redox Biol. 2, 702-714 (2014).

10. Sun, M. et al. Free radical damage in ischemia reperfusion injury: An obstacle in acute ischemic stroke after revascularization therapy. Oxid. Med. Cell. Longev. in press, (2017).

11. Khacho, M. et al. Acidosis overrides oxygen deprivation to maintain mitochondrial function and cell survival. Nat. Commun. 5, 1-15 (2014).

12. Fan, Y.-Y. et al. A novel neuroprotective strategy for ischemic stroke: transient mild acidosis treatment by $\mathrm{CO} 2$ inhalation at reperfusion. J. Cereb. Blood Flow Metab. 34, 275-283 (2013).

13. Sierra, A., Abiega, O., Shahraz, A. \& Neumann, H. Janus-faced microglia: beneficial and detrimental consequences of microglial phagocytosis. Front. Cell. Neurosci. 7, 1-22 (2013).

14. Chen, Y. et al. A novel mouse model of thromboembolic stroke. J Neurosci Methods 256, 560-574 (2015).

15. Chiang, T., Messing, R. O. \& Chou, W.-H. Mouse model of middle cerebral artery occlusion. J. Vis. Exp 48, 1-3 (2011).

16. Morris, G. P. et al. A comparative study of variables influencing ischemic injury in the Longa and Koizumi methods of intraluminal filament middle cerebral artery occlusion in mice. PLoS One 1-34 (2016). doi:10.1371/journal.pone.0148503

17. Bloise, F. F., Oliveira, T. S., Cordeiro, A. \& Ortiga-Carvalho, T. M. Thyroid hormones play role in sarcopenia and myopathies. Front. Physiol. 9, 1-7 (2018). 
18. Desgeorges, M. M. et al. Molecular mechanisms of skeletal muscle atrophy in a mouse model of cerebral ischemia. Stroke. 46, 1673-1680 (2015).

19. Basit, F. et al. Mitochondrial complex I inhibition triggers a mitophagy-dependent ROS increase leading to necroptosis and ferroptosis in melanoma cells. Nat. Publ. Gr. (2017). doi:10.1038/cddis.2017.133

20. McManus, L., Hu, X., Rymer, W. Z., Suresh, N. L. \& Lowery, M. M. Motor unit activity during fatiguing isometric muscle contraction in hemispheric stroke survivors. Front. Hum. Neurosci. 11, 1-12 (2017).

21. McNulty, P. A., Lin, G. \& Doust, C. G. Single motor unit firing rate after stroke is higher on the less-affected side during stable low-level voluntary contractions. Front. Hum.

Neurosci. 8, 1-12 (2014).

22. Thibaut, A. et al. Spasticity after stroke: Physiology, assessment and treatment. Brain Inj. 27, 1093-1105 (2013).

23. Lin, N., Liu, M. S., Fan, S. Y., Guan, Y. Z. \& Cui, L. Y. Asynchronization in changes of electrophysiology and pathology of spinal cord motor neurons in rats following middle cerebral artery occlusion. Chin. Med. J. (Engl). 128, 2919-2925 (2015).

24. Ramnemark, A., Nyberg, L., Lorentzon, R., Olsson, T. \& Gustafson, Y. Hemiosteoporosis after severe stroke, independent of changes in body composition and weight. Stroke 30, 755-760 (1999).

25. Kouzi, I. et al. Motor unit number estimation and quantitative needle electromyography in stroke patients. J. Electromyogr. Kinesiol. 24, 910-916 (2014).

26. Shepherd, R. B. Exercise and training to optimize functional motor performance in stroke: driving neural reorganization? Neural Plast. 8, 121-9 (2001).

27. Florman, J. E., Duffau, H. \& Rughani, A. I. Lower motor neuron findings after upper motor neuron injury: Insights from postoperative supplementary motor area syndrome. Front. Hum. Neurosci. 7, 1-7 (2013).

28. Nielsen, J. B., Petersen, N. T., Crone, C. \& Sinkjaer, T. Stretch reflex regulation in healthy subjects and patients with spasticity. Neuromodulation 8, 49-57 (2005).

29. Ueno, M. et al. Corticospinal circuits from the sensory and motor cortices differentially regulate skilled movements through distinct spinal interneurons. Cell Rep. 23, 1286-1300 (2018).

30. Mitchell, E. J., McCallum, S., Dewar, D. \& Maxwell, D. J. Corticospinal and reticulospinal contacts on cervical commissural and long descending propriospinal neurons in the adult rat spinal cord; evidence for powerful reticulospinal connections. PLoS One 11, 1-19 (2016).

31. Lemon, R. N. Descending pathways in motor control. Annu. Rev. Neurosci. 31, 195-218 (2008).

32. Sherman, D. et al. Anatomical location of the mesencephalic locomotor region and its possible role in locomotion, posture, cataplexy, and Parkinsonism. Front. Neurol. 6, 1-13 (2015).

33. Drew, T., Dubuc, R. \& Rossignol, S. Discharge patterns of reticulospinal and other 
reticular neurons in chronic, unrestrained cats walking on a treadmill. J. Neurophysiol. 55, 375-401 (1986).

34. Shevtsova, N. A. et al. Organization of left-right coordination of neuronal activity in the mammalian spinal cord: Insights from computational modelling. J. Physiol. 593, 24032426 (2015).

35. Talpalar, A. E. \& Kiehn, O. Glutamatergic mechanisms for speed control and network operation in the rodent locomotor CPG. Front. Neural Circuits 4, 1-14 (2010).

36. Talpalar, A. E. et al. Dual-mode operation of neuronal networks involved in left-right alternation. Nature 500, 85-8 (2013).

37. Mendelsohn, A. I. Specifying Neurons and Circuits for Limb Motor Control. (Columbia University, 2016).

38. Basso, D. M., Beattie, M. S. \& Bresnahan, J. C. A sensitive and reliable locomotor rating scale for open field testing in rats. J. Neurotrauma 12, 1-21 (1995).

39. Basso, D., Beattie, M. \& Bresnahan, J. Graded histological and locomotor outcomes after spinal cord contusion using the NYU weight-drop device versus transection. Exp. Neurol. 139, 244-56 (1996).

40. Vandeputte, C. et al. Automated quantitative gait analysis in animal models of movement disorders. BMC Neurosci. 11, 92 (2010).

41. Brown, T. G. The intrinsic factors in the act of progression in the mammal. Proc. R. Soc. B Biol. Sci. 84, 308-319 (1911).

42. Rossignol, S. Plasticity of connections underlying locomotor recovery after central and/or peripheral lesions in the adult mammals. Philos. Trans. R. Soc. Lond. B. Biol. Sci. 361, 1647-1671 (2006).

43. Saleh, M., Takahashi, K. \& Hatsopoulos, N. G. Encoding of coordinated reach and grasp trajectories in primary motor cortex. J. Neurosci. 32, 1220-1232 (2012).

44. Beloozerova, I. N., Farrell, B. J., Sirota, M. G. \& Prilutsky, B. I. Differences in movement mechanics, electromyographic, and motor cortex activity between accurate and nonaccurate stepping. J. Neurophysiol. 103, 2285-2300 (2010).

45. $\mathrm{Li}, \mathrm{X}$. Examination of post-stroke alteration in motor unit firing behavior using high density surface EMG decomposition. IEEE Trans Biomed Eng 62, 1242-1252 (2015).

46. Chou, L.-W., Palmer, J. a, Binder-Macleod, S. \& Knight, C. a. Motor unit rate coding is severely impaired during forceful and fast muscular contractions in individuals post stroke. J. Neurophysiol. 109, 2947-54 (2013).

47. Hu, X., Suresh, A. K., Rymer, W. Z. \& Suresh, N. L. Altered motor unit discharge patterns in paretic muscles of stroke survivors assessed using surface electromyography. $J$. Neural Eng. (2016). doi:10.1088/1741-2560/13/4/046025

48. Fu, Q. G., Flament, D., Coltz, J. D. \& Ebner, T. J. Temporal encoding of movement kinematics in the discharge of primate primary motor and premotor neurons. $J$. Neurophysiol. 73, 836-54 (1995).

49. Yakovenko, S. \& Drew, T. A motor cortical contribution to the anticipatory postural adjustments that precede reaching in the cat. J. Neurophysiol. 102, 853-74 (2009). 
50. Scott, S. H. \& Kalaska, J. F. Changes in motor cortex activity during reaching movements with similar hand paths but different arm postures. J. Neurophysiol. 73, 2563-7 (1995).

51. Khroyan, T. V. et al. Rodent motor and neuropsychological behaviour measured in home cages using the integrated modular platform SmartCage ${ }^{\mathrm{TM}}$. Clin. Exp. Pharmacol.

Physiol. 39, 614-622 (2012).

52. Rajasekaran, N., Tran, R., Pascual, C., Xie, X. \& Mellins, E. D. Reduced locomotor activity correlates with increased severity of arthritis in a mouse model of antibodyinduced arthritis. Open J Rheumatol Autoimmune Dis 4, 62-68 (2014).

53. Qu, W., Liu, N., Xie, X. (Simon), Li, R. \& Xu, X. Automated monitoring of early neurobehavioral changes in mice following traumatic brain injury. Neural Regen. Res. 11, 248 (2016).

54. Yakovenko, S., Boots, M., Ellison, R. \& Tuntevski, K. Walkway Device and Method for Quantitative Analysis of Gait and its Modification in Rodents. 28 (2017).

55. Tuntevski, K., Ellison, R. \& Yakovenko, S. Asymmetric walkway: A novel behavioral assay for studying asymmetric locomotion. J. Vis. Exp. JoVE e52921 (2016). doi:doi:10.3791/52921

56. Pierani, A., Brenner-Morton, S., Chiang, C. \& Jessell, T. M. A Sonic hedgehogindependent, retinoid-activated pathway of neurogenesis in the ventral spinal cord. Cell 97, 903-915 (1999).

57. Stifani, N. Motor neurons and the generation of spinal motor neuron diversity. Front. Cell. Neurosci. 8, 1-22 (2014).

58. Panovska-Griffiths, J., Page, K. M. \& Briscoe, J. A gene regulatory motif that generates oscillatory or multiway switch outputs. J. R. Soc. Interface 10, (2013).

59. Schreiber, R. et al. Retinyl ester hydrolases and their roles in vitamin A homeostasis. Biochim. Biophys. Acta 1821, 113-23 (2012).

60. Hoffman, B. G. et al. Identification of transcripts with enriched expression in the developing and adult pancreas. Genome Biol. 9, R99 (2008).

61. Chuang, C.-M. et al. Valproic acid downregulates RBP4 and elicits hypervitaminosis Ateratogenesis--a kinetic analysis on retinol/retinoic acid homeostatic system. PLoS One 7, e43692 (2012).

62. Vitins, A. P. et al. Mechanisms of amiodarone and valproic acid induced liver steatosis in mouse in vivo act as a template for other hepatotoxicity models. Arch. Toxicol. (2014). doi:10.1007/s00204-014-1211-0

63. Niederreither, K., Vermot, J., Schuhbaur, B., Chambon, P. \& Dollé, P. Retinoic acid synthesis and hindbrain patterning in the mouse embryo. Development 127, 75-85 (2000).

64. Oosterveen, T. et al. Retinoids regulate the anterior expression boundaries of 5' Hoxb genes in posterior hindbrain. EMBO J. 22, 262-9 (2003).

65. Niederreither, K., Vermot, J., Fraulob, V., Chambon, P. \& Dolle, P. Retinaldehyde dehydrogenase 2 (RALDH2)- independent patterns of retinoic acid synthesis in the mouse embryo. Proc. Natl. Acad. Sci. 99, 16111-16116 (2002). 
66. Miano, J. M. \& Berk, B. C. Retinoids: Versatile biological response modifiers of vascular smooth muscle phenotype. Circ. Res. 87, 355-362 (2000).

67. Han, Y.-H. et al. A unique cytoplasmic localization of retinoic acid receptor-gamma and its regulations. J. Biol. Chem. 284, 18503-14 (2009).

68. Chambeyron, S. \& Bickmore, W. A. Chromatin decondensation and nuclear reorganization of the HoxB locus upon induction of transcription. Genes Dev. 18, 11191130 (2004).

69. Ahn, Y., Mullan, H. E. \& Krumlauf, R. Long-range regulation by shared retinoic acid response elements modulates dynamic expression of posterior Hoxb genes in CNS development. Dev. Biol. 388, 134-44 (2014).

70. Bok, J. et al. Transient retinoic acid signaling confers anterior-posterior polarity to the inner ear. Proc. Natl. Acad. Sci. U. S. A. 108, 161-6 (2011).

71. Lin, J. et al. Directed differentiation of mouse cochlear neural progenitors in vitro. Am. J. Physiol. Cell Physiol. 296, C441-52 (2009).

72. Schulte, D. \& Frank, D. TALE transcription factors during early development of the vertebrate brain and eye. Dev. Dyn. 243, 99-116 (2014).

73. Emmett, S. D. \& West, K. P. Gestational vitamin A deficiency: a novel cause of sensorineural hearing loss in the developing world? Med. Hypotheses 82, 6-10 (2014).

74. Shim, H. J., Kang, H. H., Ahn, J. H. \& Chung, J. W. Retinoic acid applied after noise exposure can recover the noise-induced hearing loss in mice. Acta Otolaryngol. 129, 233-8 (2009).

75. Zimová-Herknerová, M., Myslivecek, J. \& Potmesil, P. Retinoic acid attenuates the mild hyperoxic lung injury in newborn mice. Physiol. Res. 57, 33-40 (2008).

76. James, M. L., Ross, a C., Bulger, A., Philips, J. B. \& Ambalavanan, N. Vitamin A and retinoic acid act synergistically to increase lung retinyl esters during normoxia and reduce hyperoxic lung injury in newborn mice. Pediatr. Res. 67, 591-7 (2010).

77. Maniscalco, W. M., Watkins, R. H., D’Angio, C. T. \& Ryan, R. M. Hyperoxic injury decreases alveolar epithelial cell expression of vascular endothelial growth factor (VEGF) in neonatal rabbit lung. Am. J. Respir. Cell Mol. Biol. 16, 557-67 (1997).

78. Nadeau, K. et al. Modulation of Lgl1 by steroid, retinoic acid, and vitamin D models complex transcriptional regulation during alveolarization. Pediatr. Res. 67, 375-381 (2010).

79. Ferrai, C. et al. Induction of Hoxb transcription by retinoic acid requires actin polymerization. Mol. Biol. Cell 20, 3543-3551 (2009).

80. Ehmann, H. et al. Time-dependent gene expression analysis of the developing superior olivary complex. J. Biol. Chem. 288, 25865-79 (2013).

81. Howell, D. M. et al. Molecular guidance cues necessary for axon pathfinding from the ventral cochlear nucleus. J. Comp. Neurol. 504, 533-549 (2007).

82. Park, J. G. et al. Loss of Mafb function in humans and mice causes Duane syndrome, aberrant extraocular muscle innervation, and inner-ear defects. Am. J. Hum. Genet. 98, 1220-1227 (2016). 
83. Stam, F. J. et al. Renshaw cell interneuron specialization is controlled by a temporally restricted transcription factor program. Development 139, 179-190 (2012).

84. Gonzalez, A. B. V1-Derived Renshaw cells and la Inhibitory Interneurons Differentiate Early During Development. Development (Wright State University, 2011).

85. Zhang, Y. \& Ross, a C. Retinoic acid and the transcription factor MafB act together and differentially to regulate aggrecan and matrix metalloproteinase gene expression in neonatal chondrocytes. J. Cell. Biochem. 114, 471-9 (2013).

86. Blosa, M. et al. Unique features of extracellular matrix in the mouse medial nucleus of trapezoid body--implications for physiological functions. Neuroscience 228, 215-34 (2013).

87. Chang, C.-W. et al. Identification of a developmentally regulated striatum-enriched zincfinger gene, Nolz-1, in the mammalian brain. Proc. Natl. Acad. Sci. U. S. A. 101, 2613-8 (2004).

88. Di Bonito, M. et al. Assembly of the auditory circuitry by a Hox genetic network in the mouse brainstem. PLoS Genet. 9, e1003249 (2013).

89. Chatonnet, F. et al. Early development of respiratory rhythm generation in mouse and chick. Respir. Physiol. Neurobiol. 131, 5-13 (2002).

90. Kabayiza, K. U. et al. The Onecut transcription factors regulate differentiation and distribution of dorsal interneurons during spinal cord development. Front. Mol. Neurosci. 10, 1-17 (2017).

91. Rottkamp, C. A., Lobur, K. J., Wladyka, C. L., Lucky, A. K. \& O'Gorman, S. Pbx3 is required for normal locomotion and dorsal horn development. Dev. Biol. 314, 23-39 (2008).

92. Cohen, D. R., Cheng, C. W., Cheng, S. H. \& Hui, C. C. Expression of two novel mouse Iroquois homeobox genes during neurogenesis. Mech. Dev. 91, 317-321 (2000).

93. Ji, S.-J., Periz, G. \& Sockanathan, S. Nolz1 is induced by retinoid signals and controls motoneuron subtype identity through distinct repressor activities. Development 136, 231240 (2009).

94. Hamburger, V. \& Hamilton, H. L. A series of normal stages in the development of the chick embryo. J. Morphol. 88, 49-92 (1951).

95. Kiehn, O. Locomotor circuits in the mammalian spinal cord. Annu. Rev. Neurosci. 29, 279-306 (2006).

96. Moran-Rivard, L. et al. Evx1 is a postmitotic determinant of V0 interneuron identity in the spinal cord. Neuron 29, 385-399 (2001).

97. Gosgnach, S. et al. V1 spinal neurons regulate the speed of vertebrate locomotor outputs. Nature 440, 215-219 (2006).

98. Bikoff, J. B. et al. Spinal inhibitory interneuron diversity delineates variant motor microcircuits. Cell 165, 207-219 (2016).

99. Zhang, J. et al. V1 and V2b interneurons secure the alternating flexor-extensor motor activity mice require for limbed locomotion. Neuron 82, 138-150 (2014). 
100. Azim, E., Jiang, J., Alstermark, B. \& Jessell, T. M. Skilled reaching relies on a V2a propriospinal internal copy circuit. Nature 508, 357-363 (2014).

101. Adams, K. L., Rousso, D. L., Umbach, J. A. \& Novitch, B. G. Foxp1-mediated programming of limb-innervating motor neurons from mouse and human embryonic stem cells. Nat. Commun. 6, 1-16 (2015).

102. Eccles, J. C. The Inhibitory Pathways of the Central Nervous System. (Charles C. Thomas, 1969).

103. Bhumbra, G. S. et al. The recurrent case for the Renshaw cell. J. Neurosci. 34, 12919-32 (2014).

104. Gabitto, M. I. et al. Bayesian sparse regression analysis documents the diversity of spinal inhibitory interneurons. Cell 165, 220-233 (2016).

105. Odden, J. P., Holbrook, S. \& Doe, C. Q. Drosophila Hb9 is expressed in a subset of motoneurons and interneurons, where it regulates gene expression and axon pathfinding. J. Neurosci. Off. J. Soc. Neurosci. 22, 9143-9149 (2002).

106. Kwan, A. C., Dietz, S. B., Webb, W. W. \& Harris-Warrick, R. M. Activity of Hb9 interneurons during fictive locomotion in mouse spinal cord. J. Neurosci. 29, 11601-13 (2009).

107. Agalliu, D., Takada, S., Agalliu, I., McMahon, A. P. \& Jessell, T. M. Motor neurons with axial muscle projections specified by Wnt4/5 signaling. Neuron 61, 708-720 (2009).

108. Caspary, T. \& Anderson, K. V. Patterning cell types in the dorsal spinal cord: What the mouse mutants say. Nat. Rev. Neurosci. 4, 289-297 (2003).

109. Bermingham, N. A. et al. Proprioceptor pathway development is dependent on MATH1. Neuron 30, 411-422 (2001).

110. Gowan, K. et al. Crossinhibitory activities of Ngn1 and Math1 allow specification of distinct dorsal interneurons. Neuron 31, 219-232 (2001).

111. Sander, M. et al. Ventral neural patterning by Nkx homeobox genes: Nkx6.1 controls somatic motor neuron and ventral interneuron fates. Genes Dev. 14, 2134-2139 (2000).

112. Lu, D. C., Niu, T. \& Alaynick, W. A. Molecular and cellular development of spinal cord locomotor circuitry. Front. Mol. Neurosci. 8, 1-18 (2015).

113. Alaynick, W. A., Jessell, T. M. \& Pfaff, S. L. SnapShot: Spinal cord development. Cell 146, 178-178.e1 (2011).

114. Vallstedt, A. \& Kullander, K. Dorsally derived spinal interneurons in locomotor circuits. Ann. N. Y. Acad. Sci. 1279, 32-42 (2013).

115. Monica, K., Galili, N., Nourse, J., Saltman, D. \& Cleary, M. L. PBX2 and PBX3, new homeobox genes with extensive homology to the human proto-oncogene PBX1. Mol. Cell. Biol. 11, 6149-57 (1991).

116. Rhee, J. W. et al. Pbx3 deficiency results in central hypoventilation. Am. J. Pathol. 165, 1343-1350 (2004).

117. Rottkamp, C. A.-M. The Role of Hox Cofactors in Vertebrate Spinal Cord Development. (Case Western Reserve University, 2008). 
118. Dyck, J., Lanuza, G. M. \& Gosgnach, S. Functional characterization of dl6 interneurons in the neonatal mouse spinal cord. J. Neurophysiol. 107, 3256-3266 (2012).

119. Gard, C. et al. Pax3- and Pax7-mediated Dbx1 regulation orchestrates the patterning of intermediate spinal interneurons. Dev. Biol. 432, 24-33 (2017).

120. Gosgnach, S. The role of genetically-defined interneurons in generating the mammalian locomotor rhythm. Integr. Comp. Biol. 51, 903-912 (2011).

121. Tripodi, M., Stepien, A. E. \& Arber, S. Motor antagonism exposed by spatial segregation and timing of neurogenesis. Nature 479, 61-66 (2011).

122. Sherrington, C. S. Flexion-reflex of the limb, crossed extension-reflex, and reflex stepping and standing. J. Physiol. 40, 28-121 (1910).

123. Jones, J. G., Tansey, E. M. T. \& Stuart, D. G. Thomas Graham Brown (1882-1965): behind the scenes at the cardiff institute of physiology. J. Hist. Neurosci. 20, 188-209 (2011).

124. Lloyd, D. P. \& Wilson, V. J. Functional organization in the terminal segments of the spinal cord with a consideration of central excitatory and inhibitory latencies in monosynaptic reflex systems. J. Gen. Physiol. 42, 1219-31 (1959).

125. Jankowska, B. Y. E., Jukes, M., Lund, S. \& Lundberg, A. The effect of DOPA on the spinal cord. Acta Physiol. Scand. 70, 389-402 (1967).

126. Stein, P. S. Intersegmental coordination of swimmeret motoneuron activity in crayfish. J. Neurophysiol. 34, 310-318 (1971).

127. Grillner, S. \& Wallén, P. How does the lamprey central nervous-system make the lamprey swim. J. Exp. Biol. 112, 337-357 (1984).

128. Heitler, W. J. Motor programme switching in the crayfish swimmeret system. J. Exp. Biol. 114, 521-49 (1985).

129. Rubin, J. E., Shevtsova, N. a, Ermentrout, G. B., Smith, J. C. \& Rybak, I. a. Multiple rhythmic states in a model of the respiratory central pattern generator. J. Neurophysiol. 101, 2146-65 (2009).

130. Close, J., Nickel, E. \& Todd, F. Motor-unit action-potential counts. J. Bone Jt. Surg. 42-A, 1207-1222 (1960).

131. Sherrington, C. S. Remarks on some aspects of reflex inhibition. Proc. R. Soc. B Biol. Sci. 97, 519-545 (1925).

132. Schiaffino, S. \& Reggiani, C. Fiber types in mammalian skeletal muscles. Physiol Rev 91, 1447-1531 (2011).

133. Augusto, V., Padovani, C. R. \& Campos, G. E. R. Skeletal muscle fiber types in C57BL6J mice. Brazilian J. Morphol. Sci. 21, 89-94 (2004).

134. Schiaffino, S. \& Reggiani, C. Myosin isoforms in mammalian skeletal muscle. J. Appl. Physiol 77, 493-501 (1994).

135. Jackman, R. W. \& Kandarian, S. C. The molecular basis of skeletal muscle atrophy. Am.J.Physiol Cell Physiol 287, C834-C843 (2004). 
136. Tintignac, L. A., Brenner, H.-R. \& Rüegg, M. A. Mechanisms regulating neuromuscular junction development and function and causes of muscle wasting. Physiol Rev 95, 809852 (2015).

137. Chung, H. T. \& Joe, Y. Antagonistic crosstalk between SIRT1, PARP-1, and -2 in the regulation of chronic inflammation associated with aging and metabolic diseases. Integr. Med. Res. 3, 198-203 (2014).

138. Peng, L. et al. Ubiquitinated Sirtuin 1 (SIRT1) function is modulated during DNA damageinduced cell death and survival. J. Biol. Chem. 290, 8904-8912 (2015).

139. Pan, M., Yuan, H., Brent, M., Ding, E. C. \& Marmorsteins, R. Sirt1 contains N- and Cterminal regions that potentiate deacetylase activity. J. Biol. Chem. 287, 2468-2476 (2012).

140. Tomita, T. et al. Sirt1-deficiency causes defective protein quality control. Sci. Rep. 5, 12613 (2015).

141. Chalkiadaki, A., Igarashi, M., Nasamu, A. S., Knezevic, J. \& Guarente, L. Muscle-specific Sirt1 gain-of-function increases slow-twitch fibers and ameliorates pathophysiology in a mouse model of Duchenne muscular dystrophy. PLoS Genet. 10, 1-12 (2014).

142. Jay, G., Khoury, G., DeLeo, A. B., Dippold, W. G. \& Old, L. J. p53 transformation-related protein: Detection of an associated phosphotransferase activity. Proc. Natl. Acad. Sci. U. S. A. 78, 2932-2936 (1981).

143. Kastan, M. B., Onyekwere, O., Sidransky, D., Vogelstein, B. \& Craig, R. W. Participation of p53 protein in the cellular response to DNA damage. Cancer Res. 5, 6304-6311 (1991).

144. Blagosklonny, M. V. p53: An ubiquitous target of anticancer drugs. Int. J. Cancer 98, 161-166 (2002).

145. Kamada, R., Toguchi, Y., Nomura, T., Imagawa, T. \& Sakaguchi, K. Tetramer formation of tumor suppressor protein p53: Structure, function, and applications. Biopolymers 106, 598-612 (2016).

146. Gabizon, R. et al. Specific recognition of $p 53$ tetramers by peptides derived from $p 53$ interacting proteins. PLoS One 7, (2012).

147. Kamada, R., Nomura, T., Anderson, C. W. \& Sakaguchi, K. Cancer-associated p53 tetramerization domain mutants: Quantitative analysis reveals a low threshold for tumor suppressor inactivation. J. Biol. Chem. 286, 252-258 (2011).

148. D'Abramo, M. et al. The p53 tetramer shows an induced-fit interaction of the C-terminal domain with the DNA-binding domain. Oncogene 35, 3272-3281 (2016).

149. Reed, S. M. \& Quelle, D. E. P53 acetylation: Regulation and consequences. Cancers (Basel). 7, 30-69 (2014).

150. Zhao, Y. et al. Acetylation of $p 53$ at Lysine $373 / 382$ by the Histone Deacetylase Inhibitor Depsipeptide Induces Expression of p21 Waf1/Cip1. Mol. Cell. Biol. 26, 2782-2790 (2006).

151. Wang, D. et al. The p53-SET interplays reveal a new mode of acetylation-dependent regulation. Nature 538, 118-122 (2016). 
152. Lavin, M. F. \& Gueven, N. The complexity of p53 stabilization and activation. Cell Death Differ. 13, 941-950 (2006).

153. Marine, J. C. p53 stabilization: The importance of nuclear import. Cell Death Differ. 17, 191-192 (2010).

154. Abida, W. M., Nikolaev, A., Zhao, W., Zhang, W. \& Gu, W. Fbxo11 promotes the neddylation of p53 and inhibits its transcriptional activity. J. Biol. Chem. 282, 1797-1804 (2007).

155. Wiman, K. Oncogenes, tumor suppressor genes and the hallmarks of cancer. (2011).

156. Stehmeier, P. \& Muller, S. Regulation of $\mathrm{p} 53$ family members by the ubiquitin-like SUMO system. DNA Repair (Amst). 8, 491-498 (2009).

157. Ito, A. et al. MDM2-HDAC1-mediated deacetylation of $\mathrm{p} 53$ is required for its degradation. $E M B O$ J. 21, 6236-6245 (2002).

158. Hori, Y. S., Kuno, A., Hosoda, R. \& Horio, Y. Regulation of FoxOs and p53 by Sirt1 modulators under oxidative stress. PLoS One 8, e73875 (2013).

159. Puigserver, P. Tissue-specific regulation of metabolic pathways through the transcriptional coactivator PGC1-a. Int. J. Obes. 29, S5-S9 (2005).

160. Patti, M. E. et al. Coordinated reduction of genes of oxidative metabolism in humans with insulin resistance and diabetes: Potential role of PGC1 and NRF1. Proc. Natl. Acad. Sci. 100, 8466-8471 (2003).

161. Arany, Z. et al. Transcriptional coactivator PGC-1a controls the energy state and contractile function of cardiac muscle. Cell Metab. 1, 259-271 (2005).

162. Rowe, G. C., El-Khoury, R., Patten, I. S., Rustin, P. \& Arany, Z. PGC-1a is dispensable for exercise-induced mitochondrial biogenesis in skeletal muscle. PLoS One 7, (2012).

163. Rasbach, K. A. et al. PGC-1 a regulates a HIF2 $\alpha$-dependent switch in skeletal muscle fiber types. Proc. Natl. Acad. Sci. 2010, 2-7 (2010).

164. Olmos, Y. et al. Mutual dependence of FoxO3a and PGC-1 $\alpha$ in the induction of oxidative stress genes. J. Biol. Chem. 284, 14476-14484 (2009).

165. Sandri, M. et al. PGC-1 $\alpha$ protects skeletal muscle from atrophy by suppressing FoxO3 action and atrophy-specific gene transcription. Proc. Natl. Acad. Sci. U. S. A. 103, 16260-5 (2006).

166. Weigel, D. \& Jäckle, H. The fork head domain: a novel DNA binding motif of eukaryotic transcription factors? Cell 63, 455-6 (1990).

167. Pani, L. et al. Hepatocyte nuclear factor 3 beta contains two transcriptional activation domains, one of which is novel and conserved with the Drosophila fork head protein. Mol. Cell. Biol. 12, 3723-32 (1992).

168. Zhu, G., Muller, E. G., Amacher, S. L., Northrop, J. L. \& Davis, T. N. A dosage-dependent suppressor of a temperature-sensitive calmodulin mutant encodes a protein related to the fork head family of DNA-binding proteins. Mol. Cell. Biol. 13, 1779-87 (1993).

169. Barr, F. G. Gene fusions involving PAX and FOX family members in alveolar rhabdomyosarcoma. Oncogene 20, 5736-5746 (2001). 
170. Gottlieb, S. \& Ruvkun, G. daf-2, daf-16 and daf-23: Genetically interacting genes controlling dauer formation in Caenorhabditis elegans. Genetics 137, 107-120 (1994).

171. Sun, X., Chen, W. D. \& Wang, Y. D. DAF-16/FOXO transcription factor in aging and longevity. Front. Pharmacol. 8, 1-8 (2017).

172. Cervenka, I., Agudelo, L. Z. \& Ruas, J. L. Kynurenines: Tryptophan's metabolites in exercise, inflammation, and mental health. Science (80-. ). 357, 1-8 (2017).

173. Xing, J. et al. Upregulation of Unc-51-like kinase 1 by nitric oxide stabilizes Sirt1, independent of autophagy. PLoS One 9, 1-20 (2014).

174. Wen, Y. C. et al. Tumor suppressor HIC1 directly regulates SIRT1 to modulate p53dependent DNA-damage responses. Cell 123, 437-448 (2005).

175. Brunet, A. et al. 14-3-3 transits to the nucleus and participates in dynamic nucleocytoplasmic transport. J. Cell Biol. 156, 817-828 (2002).

176. Schmidt, M. et al. Cell cycle inhibition by FoxO forkhead transcription factors involves downregulation of cyclin D. Mol. Cell. Biol. 22, 7842-7852 (2002).

177. Burgering, B. M. T. \& Kops, G. J. P. L. Cell cycle and death control: Long live Forkheads. Trends Biochem. Sci. 27, 352-360 (2002).

178. Putker, M. et al. Evolutionary acquisition of cysteines determines FoxO paralog-specific Redox signaling. Antioxid. Redox Signal. 22, 15-28 (2015).

179. Pomiès, P. et al. Involvement of the FoxO1/MuRF1/Atrogin-1 signaling pathway in the oxidative stress-induced atrophy of cultured chronic obstructive pulmonary disease myotubes. PLoS One 11, 1-21 (2016).

180. Shimizu, H. et al. The calcineurin-FoxO-MuRF1 signaling pathway regulates myofibril integrity in cardiomyocytes. Elife 6, 1-19 (2017).

181. Mammucari, C. et al. FoxO3 controls autophagy in skeletal muscle in vivo. Cell Metab. 6, 458-471 (2007).

182. Bertaggia, E., Coletto, L. \& Sandri, M. Posttranslational modifications control FoxO3 activity during denervation. Am. J. Physiol. Cell Physiol. 302, C587-C596 (2011).

183. Zheng, X., Xu, M. \& Fang, Q. Role of AMPKa in skeletal muscle glycometabolism regulation and adaptation in relation to sepsis. Biomed Res. Int. (2014). doi:10.1155/2014/390760

184. Sandri, M. et al. FoxO transcription factors induce the atrophy-related ubiquitin ligase Atrogin-1 and cause skeletal muscle atrophy. Cell 117, 399-412 (2004).

185. Moriscot, A. et al. MuRF1 is a muscle fiber type II associated factor and together with MuRF2 regulates type II fiber trophicity and maintenance. J. Struct. Biol. 170, 344-353 (2010).

186. McElhinny, A. S., Kakinuma, K., Sorimachi, H., Labeit, S. \& Gregorio, C. C. Musclespecific RING finger-1 interacts with titin to regulate sarcomeric M-line and thick filament structure and may have nuclear functions via its interaction with glucocorticoid modulatory element binding protein-1. J. Cell Biol. 157, 125-136 (2002).

187. Cohen, S. et al. During muscle atrophy, thick, but not thin, filament components are 
degraded by MuRF1-dependent ubiquitylation. J. Cell Biol. 185, 1083-1095 (2009).

188. Clarke, B. A. et al. The E3 ligase MuRF1 degrades myosin heavy chain protein in dexamethasone-treated skeletal muscle. Cell Metab. 6, 376-385 (2007).

189. Perera, S., Holt, M. R., Mankoo, B. S. \& Gautel, M. Developmental regulation of MURF ubiquitin ligases and autophagy proteins nbr1, p62/SQSTM1 and LC3 during cardiac myofibril assembly and turnover. Dev. Biol. 351, 46-61 (2011).

190. Bodine, S. C. \& Baehr, L. M. Skeletal muscle atrophy and the E3 ubiquitin ligases MuRF1 and MafBx/Atrogin-1. Am. J. Physiol. Endocrinol. Metab. 307, E469-84 (2014).

191. Okamoto, T., Torii, S. \& Machida, S. Differential gene expression of muscle-specific ubiquitin ligase MAFbx/Atrogin-1 and MuRF1 in response to immobilization-induced atrophy of slow-twitch and fast-twitch muscles. J. Physiol. Sci. 61, 537-546 (2011).

192. Chen, C.-T., Lin, S.-H., Chen, J.-S. \& Hsu, Y.-J. Muscle wasting in hemodialysis patients: new therapeutic strategies for resolving an old problem. ScientificWorldJournal. 2013, 643954 (2013).

193. Suzuki, N. et al. NO production results in suspension-induced muscle atrophy through dislocation of neuronal NOS. J. Clin. Invest. 117, 2468-2476 (2007).

194. Вильчинская, Н. А. Роль Амф-активируемой протеинкиназы в гипогравитационной перестройке внутриклеточных сигнальных путей в постуральной мышце млекопитающих. (Государственный научный центр - Российской Федерации Институт медико-биологических проблем РАН, 2017).

195. Perera, S., Mankoo, B. \& Gautel, M. Developmental regulation of MURF E3 ubiquitin ligases in skeletal muscle. J. Muscle Res. Cell Motil. 33, 107-122 (2012).

196. Metzger, M. B., Pruneda, J. N., Klevit, R. E. \& Weissman, A. M. RING-type E3 ligases: Master manipulators of E2 ubiquitin-conjugating enzymes and ubiquitination. Biochim. Biophys. Acta 1843, 47-60 (2014).

197. Foletta, V. C., White, L. J., Larsen, A. E., Léger, B. \& Russell, A. P. The role and regulation of MAFbx/atrogin-1 and MuRF1 in skeletal muscle atrophy. Eur. J. Physiol. 461, 325-335 (2011).

198. Gomes, M. D., Lecker, S. H., Jagoe, R. T., Navon, A. \& Goldberg, A. L. Atrogin-1, a muscle-specific F-box protein highly expressed during muscle atrophy. Proc. Natl. Acad. Sci. U. S. A. 98, 14440-5 (2001).

199. Li, Y.-P. et al. TNF- $\alpha$ acts via p38 MAPK to stimulate expression of the ubiquitin ligase Atrogin1/MAFbx in skeletal muscle. FASEB J. 19, 362-370 (2005).

200. Muller, F. L. et al. Denervation-induced skeletal muscle atrophy is associated with increased mitochondrial ROS production. Am. J. Physiol. Integr. Comp. Physiol. 293, R1159-68 (2007).

201. Csibi, A., Tintignac, L. A., Leibovitch, M. P. \& Leibovitch, S. A. elF3-f function in skeletal muscles: To stand at the crossroads of atrophy and hypertrophy. Cell Cycle 7, 16981701 (2008).

202. Lokireddy, S. et al. Identification of Atrogin-1-targeted proteins during the myostatininduced skeletal muscle wasting. Am. J. Physiol. - Cell Physiol. 303, (2012). 
203. Li, H. H. et al. Atrogin-1 inhibits Akt-dependent cardiac hypertrophy in mice via ubiquitindependent coactivation of Forkhead proteins. J. Clin. Invest. 117, 3211-3223 (2007).

204. Lynch, C. J. et al. Sirt1 undergoes alternative splicing in a novel auto-regulatory loop with p53. PLoS One 5, (2010).

205. Shah, Z. H. et al. A deacetylase-deficient Sirt1 variant opposes full-length Sirt1 in regulating tumor suppressor p53 and governs expression of cancer-related genes. Mol. Cell. Biol. 32, 704-716 (2012).

206. Deota, S. et al. Identification of a tissue-restricted isoform of Sirt1 defines a regulatory domain that encodes specificity. Cell Rep. 18, 3069-3077 (2017).

207. Solomon, J. M. et al. Inhibition of Sirt1 catalytic activity increases p53 acetylation but does not alter cell survival following DNA damage. Mol. Cell. Biol. 26, 28-38 (2006).

208. Shang, L. et al. Serum withdrawal up-regulates human Sirt1 gene expression in a p53dependent manner. J. Cell. Mol. Med. 13, 4176-4184 (2009).

209. Zhang, C. et al. HSP25 down-regulation enhanced p53 acetylation by dissociation of SIRT1 from p53 in doxorubicin-induced H9c2 cell apoptosis. Cell Stress Chaperones (2016). doi:10.1007/s12192-015-0655-3

210. Naqvi, A. et al. A single-nucleotide variation in a p53-binding site affects nutrient-sensitive human Sirt1 expression. Hum. Mol. Genet. 19, 4123-4133 (2010).

211. Bigland, B. \& Lippold, O. C. Motor unit activity in the voluntary contraction of human muscle. J. Physiol. 125, 322-35 (1954).

212. Fuglevand, a J., Macefield, V. G. \& Bigland-Ritchie, B. Force-frequency and fatigue properties of motor units in muscles that control digits of the human hand. $J$. Neurophysiol. 81, 1718-1729 (1999).

213. Debold, E. P. Potential molecular mechanisms underlying muscle fatigue mediated by reactive oxygen and nitrogen species. Front. Physiol. 6, 1-7 (2015).

214. Pronchniewicz, E., Thompson, L. V \& Thomas, D. D. Age-related decline in actomyosin structure and function. Exp. Gerontol. 42, 931-938 (2007).

215. Hafer-Macko, C. E., Ryan, A. S., Ivey, F. M. \& Macko, R. F. Skeletal muscle changes after hemiparetic stroke and potential beneficial effects of exercise intervention strategies. J. Rehabil. Res. Dev. 45, 261-272 (2008).

216. Belda-Lois, J.-M. et al. Rehabilitation of gait after stroke: a review towards a top-down approach. J. Neuroeng. Rehabil. 8, 66 (2011).

217. Hillyer, J. E. \& Joynes, R. L. A new measure of hindlimb stepping ability in neonatally spinalized rats. Behav. Brain Res. 202, 291-302 (2009).

218. Todorov, E. \& Jordan, M. I. Optimal feedback control as a theory of motor coordination. Nat. Neurosci. 5, 1226-1235 (2002).

219. Flament, D. \& Hore, J. Relations of motor cortex neural discharge to kinematics of passive and active elbow movements in the monkey. J. Neurophysiol. 60, 1268-84 (1988).

220. Mussa-Ivaldi, F. A. Do the neurons in the motor cortex encode movement direction? An 
alternative hypothesis. Neurosci. Lett. 91, 106-11 (1988).

221. Drew, T., Jiang, W., Kably, B. \& Lavoie, S. Role of the motor cortex in the control of visually triggered gait modifications. Can. J. Physiol. Pharmacol. 74, 426-42 (1996).

222. Rekik, I. Novel Mathematical Modeling Approaches to Assess Ischemic Stroke Lesion Evolution on Medical Imaging. (University of Edinburgh, 2014).

223. Russo, C. Di, Lagaert, J., Chapuisat, G. \& A, M.-A. D. A mathematical model of inflammation during ischemic stroke. ESAIM Proceedings, EDP Sci. 30, 15-33 (2010).

224. Maguire, C. C., Sieben, J. M. \& de Bie, R. A. The influence of walking-aids on the plasticity of spinal interneuronal networks, central-pattern-generators and the recovery of gait post-stroke. A literature review and scholarly discussion. J. Bodyw. Mov. Ther. 21, 422-434 (2017).

225. Castermans, T., Duvinage, M., Cheron, G. \& Dutoit, T. Towards effective non-invasive brain-computer interfaces dedicated to gait rehabilitation systems. Brain Sci. 4, 1-48 (2014).

226. Georgopoulos, A. P., Kalaska, J. F., Caminiti, R. \& Massey, J. T. On the relations between the direction of two-dimensional arm movements and cell discharge in primate motor cortex. J. Neurosci. 2, 1527-37 (1982).

227. Churchland, M. M. et al. Neural population dynamics during reaching. Nature 487, 51-6 (2012).

228. Graziano, M. The organization of behavioral repertoire in motor cortex. Annu. Rev. Neurosci. 29, 105-34 (2006).

229. MacLean, J. N. Lamina VII neurons are rhythmically active during locomotor-like activity in the neonatal rat spinal cord. 1995, (University of Manitoba, 1995).

230. Kjaerulff, O. \& Kiehn, O. Distribution of networks generating and coordinating locomotor activity in the neonatal rat spinal cord in vitro: A lesion study. J. Neurosci. 16, 5777-5794 (1996).

231. Pérez, T. et al. An intersegmental neuronal architecture for spinal wave propagation under deletions. J. Neurosci. 29, 10254-10263 (2009).

232. Prochazka, A. \& Ellaway, P. Sensory systems in the control of movement. Compr. Physiol. 2, 2615-2627 (2012).

233. Prochazka, A. \& Yakovenko, S. Predictive and reactive tuning of the locomotor CPG. 47, 474-481 (2007).

234. Yakovenko, S. Chapter 10 - A hierarchical perspective on rhythm generation for locomotor control. Progress in Brain Research 188, (Elsevier BV., 2011).

235. Beloozerova, I. N. \& Sirota, M. G. Cortically controlled gait adjustments in the cat. Ann. N. Y. Acad. Sci. 860, 550-553 (1998).

236. Farrell, B. J., Bulgakova, M. A., Sirota, M. G., Prilutsky, B. I. \& Beloozerova, I. N. Accurate stepping on a narrow path: mechanics, EMG and motor cortex activity in the cat. J. Neurophysiol. jn.00510.2014 (2015). doi:10.1152/jn.00510.2014

237. Uluç, K., Miranpuri, A., Kujoth, G. C., Aktüre, E. \& Başkaya, M. K. Focal cerebral 
ischemia model by endovascular suture occlusion of the middle cerebral artery in the rat. J. Vis. Exp. 48, 1-5 (2011).

238. Halbertsma, J. M. The stride cycle of the cat: the modelling of locomotion by computerized analysis of automatic recordings. Acta Physiol. Scand. Suppl. 521, 1-75 (1983).

239. Wang, Y., Mohamed, J. S. \& Alway, S. E. M-cadherin-inhibited phosphorylation of $\beta-$ catenin augments differentiation of mouse myoblasts. Cell Tissue Res. (2013). doi:10.1007/s00441-012-1515-4

240. Prochazka, A. \& Yakovenko, S. The neuromechanical tuning hypothesis. Prog. Brain Res. 165, 255-65 (2007).

241. Caggiano, V. et al. Midbrain circuits that set locomotor speed and gait selection. Nat. Publ. Gr. (2018). doi:10.1038/nature25448

242. Chakkalakal, J. V., Nishimune, H., Ruas, J. L., Spiegelman, B. M. \& Sanes, J. R. Retrograde influence of muscle fibers on their innervation revealed by a novel marker for slow motoneurons. Development 137, 3489-3499 (2010).

243. Carda, S., Cisari, C. \& Invernizzi, M. Sarcopenia or muscle modifications in neurologic diseases: A lexical or patophysiological difference? Eur. J. Phys. Rehabil. Med. 49, 119130 (2013).

244. Li, X., Wang, Y. C., Suresh, N. L., Rymer, W. Z. \& Zhou, P. Motor unit number reductions in paretic muscles of stroke survivors. IEEE Trans. Inf. Technol. Biomed. 15, 505-512 (2011).

245. Shah, P. K. et al. Use of quadrupedal step training to re-engage spinal interneuronal networks and improve locomotor function after spinal cord injury. Brain 136, 3362-3377 (2013).

246. Hara, Y., Masakado, Y. \& Chino, N. The physiological functional loss of single thenar motor units in the stroke patients: When does it occur? Does it progress? Clin. Neurophysiol. 115, 97-103 (2004).

247. Ramsay, J. W., Wessel, M. a, Buchanan, T. S. \& Higginson, J. S. Poststroke muscle architectural parameters of the tibialis anterior and the potential implications for rehabilitation of foot drop. Stroke Res. Treat. 2014, 948475 (2014).

248. Sobinov, A. \& Yakovenko, S. Model of a bilateral Brown-type central pattern generator for symmetric and asymmetric locomotion. J. Neurophysiol. 119, 1071-1083 (2018).

249. Beloozerova, I. N. \& Sirota, M. G. The role of the motor cortex in the control of accuracy of locomotor movements in the cat. J. Physiol. 461, 1-25 (1993).

250. Klishko, A. N., Farrell, B. J., Beloozerova, I. N., Latash, M. L. \& Prilutsky, B. I. Stabilization of cat paw trajectory during locomotion. J. Neurophysiol. 112, 1376-1391 (2014).

251. Samara, R. F. \& Currie, S. N. Crossed Commissural Pathways in the Spinal Hindlimb Enlargement Are Not Necessary for Right-Left Hindlimb Alternation During Turtle Swimming. J. Neurophysiol. 98, 2223-2231 (2007). 


\section{Appendix}

Asymmetry Index (Al) - 66,75

Akt - 37

Ascl1 - 25

ATPase - 32, 37

BBB Scale - 16

BmpR1a/b - 24

C56BL-7 - 91

Carotid artery - 9, 10, 45, 46

Chx10 - 22, 53

Central Pattern Generator (CPG) - 0 , $13,17,56,57,58,60,75,76$, $78,98,100$

Corticospinal tract $-2,3,13,14,15,18$, $28,38,41,97,102$

Cerebral Vascular Accidents - 5, 6, 11, 12,95

Dbx1 - 21, 24, 25

Dystroglycanopathies - 4

Duchenne Muscular Dystrophy - 33

DMRT3 - 26

DNA damage response pathway - 4, $32,33,37,92$

ECL - 51

Extensor carpi radialis - 64, 65

Extensor digitorum carpi- 64, 65

elF-3 - 36

EMG - 17, 18, 64, 66

Engrailed $1-21$

EPSP - 31

Etv4 - 20

Evx1-21

FKRP - 45

FoxO1 - 35, 36, 37

FoxO3a - 33, 34, 35, 36, 37, 51, 92, 93

FoxO4 - 34

Gastrocnemius - 27, 30, 50, 92, 93

GABA - 21, 26, 101

Gata2/3 - 22, 25, 53

GMEB-1 - 36

Gsh1/2 - 25
Hb9/MNR2 - 22, 24

HDAC - 19

Hamburger \& Hamilton Stage - 21

HIC1 - 37

Hox - 20, 26

Hoxa1 - 20

Hoxb1/2 - 20

Hsp25 - 37

IPO - 35

Irx - 20, 24, 25

Interstride Length (ISL) - 42, 44, 66

Leucine Charged Residue-Rich Domain $-36$

Lhx3 - 25

Leucine Zipper Domain - 36

M1 Cortex - 12, 40, 41

Mab21 - 20

Mafb - 20

MafBx/Atrogin-1 - 36, 93, 94

Mash1 - 24

Math1/Atoh1 - 24

Muscle creatine kinase - 33

Meis $2-20$

Myosin Heavy Chain - 30, 34, 37

MuRF-1 - 35, 36, 92, 93, 94

MuRF-2 - 35

MuRF-3 - 35

Ngn1/2 - 24, 25

Nkx - 24, 25

NLS - 36

Olig2/3 - 24, 25

Onecut1(HNF-6) - 20

p0-p3 - 21-25

p38- 36

p53 - 33, 36, 37, 51, 92, 93

Pax3 - 25, 26, 34

Pax6 - 25

Pbx1 - 20, 25

$\mathrm{Pb} \times 3 \mathrm{c}-25$ 
pd1-6 - 26

pdIL -26

PGC1a - 32, 33, 34, 37, 51, 92, 93

PLA - 47, 48

pMN - 21, 22, 25

PPARY - 32, 33, 34, 37, 48

Prep1 - 20

Retinoic Acid - 21, 22, 23, 24

Raldh - 20

RAR - 19

RARE - 19, 20

Renshaw Cell - 20, 22, 26, 27, 53

RNAPII - 20

ROS -7

$\mathrm{RXR}-19$

Shh - 19, 23, 24
Shox2 - 26

Sirt1 - 32, 33, 35, 36, 37, 43, 51, 92, 93

Stride Length $-41,42,43,44,63,64$, 65,86

Soleus muscle - 50, 93

Tibialis anterior - 27, 38, 50, 92, 93

TGF- $\beta-23,24$

TIF $1 \beta-37$

tMCAO - 6, 9, 10, 11, 17, 33, 38, 91

Tissue Plasminogen Activator - 6

Triphenyltetrazolium chloride -11

V0-V3 - 14, 21, 22, 26

Znf503/Nolz1 - 20

Zpfm2 - 20

a-Motor neuron - 0, 3, 7, 8, 12, 13, 14, $21,22,23,24,26,27,29,30$, $31,32,37,40,53,94,95,98$ 